\title{
Competing orders in a magnetic field: Spin and charge order in the cuprate superconductors
}

\section{Citation}

Zhang, Ying, Eugene Demler, and Subir Sachdev. 2002. "Competing Orders in a Magnetic Field: Spin and Charge Order in the Cuprate Superconductors." Physical Review B 66 (9) (September 3). doi:10.1103/physrevb.66.094501.

\section{Published Version}

doi:10.1103/PhysRevB.66.094501

\section{Permanent link}

http://nrs.harvard.edu/urn-3:HUL.InstRepos:27945842

\section{Terms of Use}

This article was downloaded from Harvard University's DASH repository, and is made available under the terms and conditions applicable to Other Posted Material, as set forth at http:// nrs.harvard.edu/urn-3:HUL.InstRepos:dash.current.terms-of-use\#LAA

\section{Share Your Story}

The Harvard community has made this article openly available.

Please share how this access benefits you. Submit a story.

Accessibility 


\title{
Competing orders in a magnetic field: Spin and charge order in the cuprate superconductors
}

\author{
Ying Zhang* \\ Department of Physics, Yale University, P.O. Box 208120, New Haven, Connecticut 06520-8120 \\ Eugene Demler ${ }^{\dagger}$ \\ Department of Physics, Harvard University, Cambridge, Massachusetts 02138 \\ Subir Sachdev \\ Department of Physics, Yale University, P.O. Box 208120, New Haven, Connecticut 06520-8120
}

(Received 21 December 2001; revised manuscript received 18 April 2002; published 3 September 2002)

\begin{abstract}
We describe two-dimensional quantum spin fluctuations in a superconducting Abrikosov flux lattice induced by a magnetic field applied to a doped Mott insulator. Complete numerical solutions of a self-consistent large$N$ theory provide detailed information on the phase diagram and on the spatial structure of the dynamic spin spectrum. Our results apply to phases with and without long-range spin-density-wave order, and to the magnetic quantum critical point separating these phases. We discuss the relationship of our results to a number of recent neutron-scattering measurements on the cuprate superconductors in the presence of an applied field. We compute the pinning of static charge order by the vortex cores in the "spin-gap" phase where the spin order remains dynamically fluctuating, and argue that these results apply to recent scanning-tunneling-microscopy (STM) measurements. We show that, with a single typical set of values for the coupling constants, our model describes the field dependence of the elastic-neutron-scattering intensities, the absence of satellite Bragg peaks associated with the vortex lattice in existing neutron-scattering observations, and the spatial extent of charge order in STM observations. We mention implications of our theory for NMR experiments. We also present a theoretical discussion of more exotic states that can be built out of the spin- and charge-order parameters, including spin nematics and phases with "exciton fractionalization."
\end{abstract}

DOI: 10.1103/PhysRevB.66.094501

\section{INTRODUCTION}

The determination of the ground state of the cuprate superconductors as a function of the hole density has been one of the central problems in condensed matter physics in the last decade. At zero hole density, it is well established that the ground state is a Mott insulator with long-range magnetic Néel order. At moderate hole density, it is also widely accepted that the ground state is a $d$-wave superconductor, all of whose important qualitative properties are identical those of the standard BCS-BdG theory. At issue are the ground states which interpolate between these well understood limits, and the manner in which they influence the anomalous properties at temperatures $(T)$ above $T_{c}$ (the critical temperature for the onset of superconductivity).

While a plethora of interesting proposals for these intermediate states have been made, we will focus here on (in our view) the simplest possibility: the order parameters characterizing the intermediate ground states are simply those of spin- and charge-density waves (SDW and CDW), and superconductivity (SC) itself. Apart from a small range at very low doping, which shall not be of interest in this paper, we know from neutron-scattering experiments that there is SDW order collinearly polarized at the wave vectors

$$
\mathbf{K}_{s x}=\left(\frac{2 \pi}{a}\right)\left(\frac{1}{2}-\vartheta, \frac{1}{2}\right), \quad \mathbf{K}_{s y}=\left(\frac{2 \pi}{a}\right)\left(\frac{1}{2}, \frac{1}{2}-\vartheta\right),
$$

where $a$ is square lattice spacing and the wave vector shift from two sublattice order $0<\vartheta<1 / 2$ is a function of the doping concentration. In particular, strong motivation for our study here was provided by the remarkable experiments of Wakimoto et al. ${ }^{1,2}$ They showed that the onset of superconductivity in $\mathrm{La}_{2-\delta} \mathrm{Sr}_{\delta} \mathrm{CuO}_{4}$ occurs first at $\delta=0.055$ (in a first-order insulator-to-superconductor transition) into a state which also has long-range spin-density-wave order at $T=0$ with a wave vector of the form (1.1), i.e., as $\delta$ is moved away from the insulator at $\delta=0$, the first conducting state is a $\mathrm{SC}+\mathrm{SDW}$ state. As the ground state for large enough $\delta$ is an SC state, it follows that there must be at least one quantum phase transition between the SC+SDW and SC states, and we will work with the simplest possibility that there is one direct transition at some critical $\delta=\delta_{c}$. Wakimoto et al. also showed that such a transition associated with the vanishing of the SDW moment occurred for $\delta_{c} \approx 0.14$ (see Fig. 1 in Ref. 2). We shall assume that the SC+SDW to SC quantum phase transition is second-order: direct evidence for critical magnetic fluctuations in $\mathrm{La}_{2}{ }_{\delta} \mathrm{Sr}_{\delta} \mathrm{CuO}_{4}$ for $\delta \approx 0.14$ was provided in the neutron-scattering experiments of Aeppli et al. ${ }^{3}$

We will also discuss the appearance of local and longrange CDW order in the above phases. It is important to note that, throughout this paper, we use the term "charge-density wave" (or "charge order") in its most general sense: such order implies that there is a periodic spatial modulation in all observables which are invariant under spin rotations and time reversal, such as the electron kinetic energy, the exchange energy, or even the electron pairing amplitude. The modulation in the site charge density may well be unobservably small because of screening by the long-range Coulomb interactions. 
We note that the doping dependence of the magnetic order in the cuprates can be quite complex, varies significantly between different compounds, and is influenced by the degree of disorder: the magnetic order may well be spin-glasslike at the lowest energy scales at some $\delta$. The SDW order is also enhanced in the vicinity of special commensurate values of the doping such as $\delta=1 / 8$ (see, e.g., Fig. 1 in Ref. 2), along with a suppression of SC order. In general, we do not wish to enter into most of these complexities here, although we will mention (in Sec. I A) how our theory could be extended to explain the commensuration effects-some other relevant issues will be discussed in Sec. VII. Our primary assumption is that the low-energy collective excitations can be described using the theory of the vicinity of a quantum critical point between the SC+SDW and the SC phases; evidence supporting this assumption was also reviewed in Ref. 4. This critical point is present either as a function of $\delta$ in the material under consideration, or in a generalized parameter space but quite close to the physical axis.

It is also important not to confuse this magnetic quantum critical point, with other proposals for quantum critical points near optimal doping that have appeared in the recent literature. ${ }^{5,6}$ These latter critical points are near $\delta \approx 0.19$, and are probably not associated with long-range spin-density wave order at a wave vector of the form (1.1). This paper will discuss magnetic transitions at smaller doping.

Upon accepting the existence of a second order quantum critical point at $T=0$ between the SC $+\mathrm{SDW}$ and SC phases, a powerful theoretical tool for the analysis of experiments becomes available. ${ }^{7}$ The structure of the critical theory, and its associated classification of eigenperturbations, allows a systematic and controlled theory of the spin excitations in the SC and SDW phases on either side of the critical point. Such an approach was recently exploited to study the influence of nonmagnetic $\mathrm{Zn}$ and $\mathrm{Li}$ impurities in the SC phase. ${ }^{8}$ In this paper we will use the same tools to study the influence of an applied magnetic field, oriented perpendicular to the $\mathrm{CuO}_{2}$ layers, on both the SC and the SC+SDW phases. An outline of our results has already appeared in previous communications: ${ }^{9-11}$ here we will present the full numerical solution of the our self-consistent equations for the dynamic spin spectrum in an applied field, along with a number of results. Measurements of the spin and charge correlations in the presence of such an applied magnetic field have appeared recently in a number of illuminating neutron-scattering, ${ }^{12-14}$ NMR, ${ }^{15-17}$ and STM experiments, ${ }^{18}$ and we will compare their results with our prior predictions.

\section{A. Order parameters and field theory}

The field theory for a SC to SC+SDW transition in zero applied magnetic field can be expressed entirely in terms of the SDW order parameter which we will introduce in this subsection; the quantum fluctuations of the SC order can be safely neglected, a point we will discuss further in Sec. VII. Consideration of the applied magnetic field will appear in the following subsection.
We introduced above the wave vectors of the SDW ordering $\mathbf{K}_{s x}$ and $\mathbf{K}_{s y}$; almost all of our analysis will apply for general values of $\vartheta$, but the value $\vartheta=1 / 8$ is of particular interest above a doping of about 1/8. To obtain an order parameter for such a SDW, we write the spin operator $S_{\alpha}(\mathbf{r}, \tau), \alpha=x, y, z$, at the lattice site $\mathbf{r}$ as

$$
S_{\alpha}(\mathbf{r}, \tau)=\operatorname{Re}\left[e^{i \mathbf{K}_{s x} \cdot \mathbf{r}} \Phi_{x \alpha}(\mathbf{r}, \tau)+e^{i \mathbf{K}_{s y} \cdot \mathbf{r}} \Phi_{y \alpha}(\mathbf{r}, \tau)\right],
$$

where $\Phi_{x, y \alpha}$ are the required order parameters. Except for the case of two sublattice order with $\vartheta=0$ (which we exclude for now), the fields $\Phi_{x, y \alpha}$ are complex. These fields can describe a wide variety of SDW configurations, but we now list the two important limiting cases.

(i) Collinearly polarized SDW's, for which

$$
\Phi_{y \alpha}(\mathbf{r}, \tau)=e^{i \theta(\mathbf{r}, \tau)} n_{\alpha}(\mathbf{r}, \tau),
$$

where $n_{\alpha}$ is a real vector and $\theta$ is also real (and similarly for $\Phi_{x \alpha}$ ). Parametrized in this manner, and for $n_{\alpha}^{2}=$ const (summation over the repeated index $\alpha$ is implied here and henceforth), the order parameter $\Phi_{y \alpha}$ belongs to the space $\left(S_{2}\right.$ $\left.\times S_{1}\right) / Z_{2}$, where $S_{n}$ is the $n$-dimensional surface of a sphere in $n+1$ dimensions, and $Z_{p}$ is the discrete cyclic group of $p$ elements. The $Z_{2}$ quotient is necessary because a shift $\theta$ $\rightarrow \theta+\pi$ is equivalent to a rotation which sends $n_{\alpha} \rightarrow-n_{\alpha}$.

(ii) Circular spiral SDW's, for which

$$
\Phi_{y \alpha}(\mathbf{r}, \tau)=n_{1 \alpha}(\mathbf{r}, \tau)+i n_{2 \alpha}(\mathbf{r}, \tau),
$$

where $n_{1,2 \alpha}$ are two real vectors obeying $n_{1 \alpha}^{2}=n_{2 \alpha}^{2}$ and $n_{1 \alpha} n_{2 \alpha}=0$ (and similarly for $\Phi_{x \alpha}$ ). Now for $n_{1 \alpha}^{2}=$ const, the order parameter $\Phi_{y \alpha}$ belongs to the space $\mathrm{SO}(3) \cong S_{3} / Z_{2}$ (see e.g., Sec. 13.3.2 in Ref. 7).

The experimental evidence ${ }^{19,20}$ supports the conclusion the SDW ordering in the cuprates in collinear, but the present formalism allows a common treatment of both the collinear and spiral cases. This complex-vector formulation of the SDW order allows treatment of the SDW quantum transition by a straightforward generalization of the real-vector theory used for the Néel state in the insulator; related points have been made by Castro Neto and Hone ${ }^{21}$ and Zaanen. ${ }^{22}$ The same approach was also used by Zachar et al..$^{23}$ to treat the onset of SDW order at finite temperatures, as we will indicate below.

Along with the SDW order, CDW order may also appear. We parameterize the charge density modulation by

$$
\delta \rho(\mathbf{r}, \tau)=\operatorname{Re}\left[e^{i \mathbf{K}_{c x} \cdot \mathbf{r}} \phi_{x}(\mathbf{r}, \tau)+e^{i \mathbf{K}_{c y} \cdot \mathbf{r}} \phi_{y}(\mathbf{r}, \tau)\right],
$$

where $\mathbf{K}_{c x, y}$ are the CDW ordering wave vectors and $\phi_{x, y}$ the corresponding complex order parameters. The quantum numbers of the observable $\delta \rho$ are identical to those of $S_{\alpha}^{2}$, and so by squaring Eq. (1.2) we see that associated with the SDW is a CDW with $^{23} \mathbf{K}_{c x}=2 \mathbf{K}_{s x}, \mathbf{K}_{c y}=2 \mathbf{K}_{s y}$ (modulo reciprocal lattice vectors) 


$$
\phi_{x}(\mathbf{r}, \tau) \propto \Phi_{x \alpha}^{2}(\mathbf{r}, \tau) \quad \text { and } \quad \phi_{y}(\mathbf{r}, \tau) \propto \Phi_{y \alpha}^{2}(\mathbf{r}, \tau)
$$

Note that this CDW is absent for the case of a circular spiral SDW (in which case $\Phi_{x, y \alpha}^{2}=0$ ) but is necessarily present for a collinear SDW. In principle, in a state with condensates of both $\Phi_{x \alpha}$ and $\Phi_{y \alpha}$, a CDW can also be present at wave vector $\mathbf{K}_{s x}+\mathbf{K}_{s y}$; we will not consider this possibility here as it does not seem to be experimentally relevant. As was emphasized in the third paragraph of Sec. I, we are using the term CDW here in its broadest sense: there is a modulation at the wave vector $\mathbf{K}_{c}$ in all observables which are invariant under spin rotations and time reversal. The precise nature of the CDW order may be determined from an analysis of the STM spectrum-this has been discussed recently in Refs. 24,25 .

The order parameters $\Phi_{x, y \alpha}, \phi_{x, y}$ allow a rich variety of phases and phase transitions in the presence of background SC order. These will be discussed in some detail in Sec. VI. Central to a description of these phases is an understanding of the symmetries respected by any effective action for the order parameters. We describe these below and then focus on a particular phase transition of physical interest.

An obvious symmetry is that under spin rotations; this is described by the group SU(2), and the fields $\Phi_{x, y \alpha}$ transform as $S=1$ vectors labeled by the index $\alpha$. In addition, there is an independent sliding symmetry

$$
\Phi_{x, y \alpha} \rightarrow e^{i \theta_{x, y}} \Phi_{x, y \alpha}
$$

associated with the translational symmetry of the underlying lattice model: translating $\mathbf{r}$ to $\mathbf{r}+(m a, 0)(m$ integer) in Eq. (1.2) leads to Eq. (1.7) with $\theta_{x}=m \pi(1-2 \vartheta)$ and $\theta_{y}=m \pi[$ $\vartheta$ was defined in Eq. (1.1)]. For $\vartheta$ irrational, we see that all real values of $\theta_{x, y}$ can be generated with the different choices for $m$, and hence the sliding symmetry is $\mathrm{U}(1) \times \mathrm{U}(1)$. For rational $\vartheta$, with $1 / 2-\vartheta=p^{\prime} / p$, and $p^{\prime}, p$ relatively prime integers, only integer multiples of $\theta_{x, y}=2 \pi / p$ are allowed in Eq. (1.7); in this case the sliding symmetry is reduced to $Z_{p} \times Z_{p}$. The difference between $\mathrm{U}(1)$ and $Z_{p}$ will not be material to any of our results for $p>2$. In a similar manner, we can also determine the action of other elements of the square lattice space group on $\Phi_{x, y \alpha}$ and we mention two important cases: under a spatial inversion we have $\Phi_{x, y \alpha}$ $\rightarrow \Phi_{x, y \alpha}^{*}$, and under the interchange of $x$ and $y$ axes, we have $\Phi_{x \alpha} \leftrightarrow \Phi_{y \alpha}$.

We now apply these symmetries to determine the effective action of a physically relevant transition discussed earlier in the introduction (and in the phase diagrams of Sec. II): that between the SC+SDW and SC phases. This transition is driven by the condensation of $\Phi_{x, y \alpha}$; if the SDW order is collinear, it will drive a concomitant CDW order, as discussed above. Supplementing the symmetries by a renormalization group (RG) procedure which selects terms with smaller powers of $\Phi_{x, y \alpha}$ and fewer spatial and temporal gradients, we obtain ${ }^{10,11,23,26}$ the effective action

$$
\begin{aligned}
\mathcal{S}_{\Phi}= & \int d^{2} r d \tau\left[\left|\partial_{\tau} \Phi_{x \alpha}\right|^{2}+v_{1}^{2}\left|\partial_{x} \Phi_{x \alpha}\right|^{2}+v_{2}^{2}\left|\partial_{y} \Phi_{x \alpha}\right|^{2}\right. \\
& +\left|\partial_{\tau} \Phi_{y \alpha}\right|^{2}+v_{2}^{2}\left|\partial_{x} \Phi_{y \alpha}\right|^{2}+v_{1}^{2}\left|\partial_{y} \Phi_{y \alpha}\right|^{2}+s\left(\left|\Phi_{x \alpha}\right|^{2}\right. \\
& \left.+\left|\Phi_{y \alpha}\right|^{2}\right)+\frac{u_{1}}{2}\left(\left|\Phi_{x \alpha}\right|^{4}+\left|\Phi_{y \alpha}\right|^{4}\right)+\frac{u_{2}}{2}\left(\left|\Phi_{x \alpha}^{2}\right|^{2}\right. \\
& \left.+\left|\Phi_{y \alpha}^{2}\right|^{2}\right)+w_{1}\left|\Phi_{x \alpha}\right|^{2}\left|\Phi_{y \alpha}\right|^{2}+w_{2}\left|\Phi_{x \alpha} \Phi_{y \alpha}\right|^{2} \\
& \left.+w_{3}\left|\Phi_{x \alpha}^{*} \Phi_{y \alpha}\right|^{2}\right] .
\end{aligned}
$$

Note that first-order temporal gradient terms such as $\Phi_{x \alpha}^{*} \partial_{\tau} \Phi_{x \alpha}$ are forbidden by spatial inversion symmetry. ${ }^{26}$ In principle, first-order spatial gradient terms such as $i \Phi_{x \alpha}^{*} \partial_{x} \Phi_{x \alpha}$ are permitted by all symmetries; such terms lead to a shift in the wave vector at which SDW fluctuations are largest, and we assume that they have already been absorbed by our choice of $\mathbf{K}_{s x}$. Here $v_{1}$ and $v_{2}$ are velocities, which are expected to be of order the spin-wave velocity $v$ of the Néel state in the undoped insulator. The parameter $s$ tunes the system from the SC phase $\left(s>s_{c}\right)$ to the SC+SDW phase $\left(s<s_{c}\right)$, where $s=s_{c}$ is the nonuniversal location of the quantum critical point between these phases; experimentally, $s$ can be varied by changing the doping concentration. The action also contains a number of quartic nonlinearities: the RG analysis shows that these are strongly relevant perturbations about the Gaussian theory, and will play a crucial role in our analysis below. The coupling $u_{2}$ selects between the collinear and spiral SDW states: for $u_{2}>0$, the circular spiral state (which has $\Phi_{x \alpha}^{2}=0$ ) is selected, while $u_{2}<0$ prefers a collinear SDW. The couplings $w_{1,2,3}$ lead to correlations between the orders at $\mathbf{K}_{s x}$ and $\mathbf{K}_{s y}$-if these are attractive, the $s<s_{c}$ phase will have simultaneous orderings at both wave vectors, and spatial pattern will have a checkerboard structure.

We have also neglected the couplings to the low energy nodal quasiparticles, which are additional excitations of the SC phase carrying spin; their effects are suppressed by the constraints of momentum conservation, as they can damp the $\Phi$ quanta effectively only if $\mathbf{K}_{s x, y}$ equal the separation between any two nodal points. The case where this nesting condition is satisfied has been considered earlier, ${ }^{26}$ but we will not enter into it here for simplicity: essentially all of our results here on the phase diagram in an applied magnetic field apply also to the case where the nesting condition is obeyed. For completeness, in Appendix A we also discuss the role of spin symmetry breaking Dzyaloshinskii-Moriya interaction present in $\mathrm{La}_{2}-{ }_{\delta} \mathrm{Sr}_{\delta} \mathrm{CuO}_{4} \cdot{ }^{27} \mathrm{We}$ show that it helps stabilize collinear SDW order in a certain direction; however, its effect is very small and will be neglected in the rest of this paper.

For the particular rational value $\vartheta=1 / 8$, the $U(1)$ $\times \mathrm{U}(1)$ sliding symmetry is reduced to a discrete $Z_{8} \times Z_{8}$ symmetry under which $\theta_{x, y}$ in Eq. (1.7) are only allowed to be multiples of $\pi / 4$. This reduced symmetry allows additional terms in Eq. (1.8) whose structure has been discussed earlier. $^{11,23}$ Such terms help choose between site- and bondcentered density waves, ${ }^{11}$ and could also lead to the enhance- 
ment of the moment observed by Wakimoto et al. ${ }^{2}$ near $\delta$ $=1 / 8$. However, these terms are very high order (eighth) in the $\Phi$ fields, and consequently they have a negligible effect on the issues we are interested in here: so we will not consider them further.

It is useful to compare our treatment here of the SC $+\mathrm{SDW}$ to SC transition with others in the literature. It is essential for our purposes that the spin/charge ordering is taking place in a background of $\mathrm{SC}$ order, as that gaps out the fermionic excitations except possibly at special points in the Brillouin zone. Theories ${ }^{28,29}$ which consider SDW/CDW order in a Fermi liquid have additional damping terms in their effective action which change the universality class of the transition, change the dynamic exponent to $z=2$, and do not obey strong hyperscaling properties as the quartic couplings are marginally irrelevant in this case. We have also taken a genuinely two-dimensional view on the SDW/CDW ("stripe") fluctuations in our approach. An alternative approach $^{30}$ assumes there are intermediate scales on which the physics of the one-dimensional electron gas applies, although a crossover to similar two-dimensional physics occurs on large enough scales. ${ }^{31}$

\section{B. Influence of an applied magnetic field}

An applied magnetic field has a Zeeman coupling to the spin of the electrons, and this is present for any direction of the applied field. However, the Zeeman splitting of the magnetic levels has only a minor effect, and can be safely neglected compared to the much stronger effects near $s=s_{c}$ that we consider below. We discuss the influence of the Zeeman term in Appendix B, and will not consider it further in this paper.

The dominant effect of the field is via its coupling to the orbital motion of the electrons, which is sensitive only to the component of the field orthogonal to the layers. The reason for this strong effect is simple: there is SC order in the orbital wave function of the electrons, and the diamagnetic susceptibility of the SC state to the applied field is infinite. However, as the SC order is noncritical across the transition at $s=s_{c}$, it is mainly a quiescent spectator and its response can justifiably be treated in a static, mean-field theory. Consequently, we model the complex SC order parameter $\psi(\mathbf{r})$ in the familiar Abrikosov theory with the free energy per layer (we use units with $\hbar=k_{B}=1$ throughout)

$$
\begin{aligned}
\mathcal{F}= & \int d^{2} r\left[-\alpha|\psi(\mathbf{r})|^{2}+\frac{\beta}{2}|\psi(\mathbf{r})|^{4}+\frac{1}{2 m^{*}}\right. \\
& \left.\times\left|\left(\frac{1}{i} \nabla_{\mathbf{r}}-\frac{e^{*}}{c} \mathbf{A}\right) \psi(\mathbf{r})\right|^{2}\right] .
\end{aligned}
$$

Note that unlike $\Phi_{x, y \alpha}, \psi$ is not a fluctuating variable, and described completely by its mean value (which will be $\mathbf{r}$ dependent). We will work entirely in the limit of extreme type-II superconductivity (with Ginzburg-Landau parameter $\kappa_{\mathrm{GL}}=\infty$ ); so there is no screening of the magnetic field by the Meissner currents, and $\boldsymbol{\nabla}_{\mathbf{r}} \times \mathbf{A}=H \hat{z}$, the applied, spaceindependent magnetic field.
To complete the description of the model studied in this paper, we now need to couple the SC and SDW order parameters together. The simplest term allowed by symmetry is a connection between the local modulus of the order parameters

$$
\mathcal{S}_{\Phi \psi}=\kappa \int d^{2} r d \tau|\psi(\mathbf{r})|^{2}\left[\left|\Phi_{x \alpha}(\mathbf{r}, \tau)\right|^{2}+\left|\Phi_{y \alpha}(\mathbf{r}, \tau)\right|^{2}\right] .
$$

For $\kappa>0$, we can induce a competition between the SC and SDW orders, in that the SDW order will be enhanced where the SC order is suppressed and vice versa. The microscopic origin of the coupling $\kappa$ is discussed in Appendix C.

Although $\mathcal{S}_{\Phi \psi}$ will be the primary coupling between the SDW and SC orders, an additional allowed term will be important for some purposes. ${ }^{11}$ To understand this, notice that all terms in $\mathcal{S}_{\Phi}$ and $\mathcal{S}_{\Phi \psi}$ are invariant under the sliding symmetry (1.7). This means that, with the present terms, the CDW order is free to slide arbitrarily with respect to any vortex lattice that may be present in the SC order $\psi$. This clearly cannot be true, as lattice scale effects should pin the two modulations with respect to each other. The simplest additional coupling which will provide this pinning can be deduced by noticing that there should be a coupling between the charge modulation in Eq. (1.5) and the local modulus of the superconducting order; this is induced by the term ${ }^{11}$

$$
\begin{aligned}
\widetilde{\mathcal{S}}_{\text {lat }}= & -\widetilde{\zeta} \sum_{\mathbf{r}} \int d \tau|\psi(\mathbf{r})|^{2} \operatorname{Re}\left[e^{i \mathbf{K}_{c x} \cdot \mathbf{r}} \Phi_{x \alpha}^{2}(\mathbf{r}, \tau)\right. \\
& \left.+e^{i \mathbf{K}_{c y} \cdot \mathbf{r}} \Phi_{y \alpha}^{2}(\mathbf{r}, \tau)\right] .
\end{aligned}
$$

Notice that we are now performing a discrete summation over the lattice sites $\mathbf{r}$, rather than integrating over a spatial continuum: this is a direct consequence of the rapidly oscillating factors $e^{i \mathbf{K}_{c x} \cdot \mathbf{r}}$ and $e^{i \mathbf{K}_{c y} \cdot \mathbf{r}}$ which do not have a smooth continuum limit. Indeed, in regions where $\psi(\mathbf{r})$ is smoothly varying, these rapidly oscillating factor will cause the summation over $\mathbf{r}$ to vanish. So the expression (1.11) is appreciable only over regions where $\psi(\mathbf{r})$ is rapidly varying, and this happens only in the cores of the vortices. As the centers of the vortices are identified by the zeros of $\psi(\mathbf{r})$, and we are mainly interested in scales larger than vortex core size, we can replace Eq. (1.11) by the following expression, which is more amenable to an analysis in the continuum theory: ${ }^{11}$

$$
\mathcal{S}_{\text {lat }}=-\zeta \sum_{\mathbf{r}_{v}, \psi\left(\mathbf{r}_{v}\right)=0} \int d \tau \operatorname{Re}\left\{e^{i \varpi}\left[\Phi_{x \alpha}^{2}\left(\mathbf{r}_{v}, \tau\right)+\Phi_{y \alpha}^{2}\left(\mathbf{r}_{v}, \tau\right)\right]\right\}
$$

Here the summation is over all points $\mathbf{r}_{v}$ at which $\psi\left(\mathbf{r}_{v}\right)$ $=0$ (these are the centers of the vortices), and $\varpi$ is a phase which depends upon the microscopic structure of the vortex core on the lattice scale. The action $\mathcal{S}_{\text {lat }}$ is not invariant under the sliding symmetry, and so will pin the CDW order.

We are now in a position to succinctly state the fieldtheoretic problem which will be addressed in this paper. We are interested in the partition function for SDW/CDW fluctuations defined by 


$$
\begin{aligned}
\mathcal{Z}[\psi(\mathbf{r})]= & \int \mathcal{D} \Phi_{x \alpha}(\mathbf{r}, \tau) \mathcal{D} \Phi_{y \alpha}(\mathbf{r}, \tau) \\
& \times \exp \left(-\frac{\mathcal{F}}{T}-\mathcal{S}_{\Phi}-\mathcal{S}_{\Phi \psi}-\mathcal{S}_{\text {lat }}\right),
\end{aligned}
$$

accompanied by the solution of

$$
\frac{\delta \ln \mathcal{Z}[\psi(\mathbf{r})]}{\delta \psi(\mathbf{r})}=0
$$

which minimizes $-\ln \mathcal{Z}[\psi(\mathbf{r})]$ to determine the optimum $\psi(\mathbf{r})$. Note the highly asymmetrical treatment of the SDW and SC orders: we include full quantum-mechanical fluctuations of the former, while the latter is static and nonfluctuating. This asymmetry is essentially imposed on us by the perspective of magnetic quantum criticality, and the fact that we are developing a theory of the SC+SDW to SC transition. This asymmetry should also be contrasted with the symmetric treatment of SC and SDW quantum fluctuations in other approaches. ${ }^{32}$

\section{Physical discussion}

The primary purpose of this paper is to determine the phase diagram and low-energy spectrum of SDW and CDW fluctuations of $\mathcal{Z}$ as a function of the applied field $H$. A summary of our results has already appeared ${ }^{9-11}$ and detailed numerical solutions appear in the body of the paper; here we expand on the central physical idea to provide an intuitive understanding of our results to readers who do not wish to study the details in the remainder of the paper. We will initially ignore the pinning described by $\mathcal{S}_{\text {lat }}$, but will discuss its consequences in Sec. I C 1.

Let us begin in the SC phase with $s>s_{c}$ and consider the $\Phi_{x \alpha}$ fluctuations in a simple Gaussian theory (the considerations of this subsection apply equally to $\Phi_{y \alpha}$, which we will not mention further). Assume $\psi(\mathbf{r})$ has been determined by the minimization of $\mathcal{F}$, and so takes the standard form in an Abrikosov flux lattice. The Gaussian fluctuations of $\Phi_{x \alpha}$ are described by the effective action

$$
\begin{aligned}
\mathcal{S}_{G}= & \int d^{2} r d \tau\left[\left|\partial_{\tau} \Phi_{x \alpha}\right|^{2}+v_{1}^{2}\left|\partial_{x} \Phi_{x \alpha}\right|^{2}\right. \\
& \left.+v_{2}^{2}\left|\partial_{y} \Phi_{x \alpha}\right|^{2}+\mathcal{V}(\mathbf{r})\left|\Phi_{x \alpha}\right|^{2}\right] .
\end{aligned}
$$

To leading order, the effective potential $\mathcal{V}(\mathbf{r})$ is given by $\mathcal{V}$ $=\mathcal{V}_{0}$, where

$$
\mathcal{V}_{0}(\mathbf{r})=s+\kappa|\psi(\mathbf{r})|^{2}
$$

A sketch of the spatial structure of $\mathcal{V}_{0}(\mathbf{r})$ is shown in Fig. 1: because $\psi(\mathbf{r})$ vanishes at the centers of the vortices, $\mathcal{V}_{0}(\mathbf{r})$ has well-developed minima at each such point. Indeed, there can even be regions in each vortex core where $\mathcal{V}_{0}(r)<0$, and Arovas et $a l .{ }^{33}$ and Bruus et al. ${ }^{34}$ argued that superconductivity would "rotate" or transform into static Néel order in such a region. In our treatment of dynamic SDW, 9,35 we see that the structure of the magnetism is determined by the solution of the Schrödinger equation ${ }^{36}$

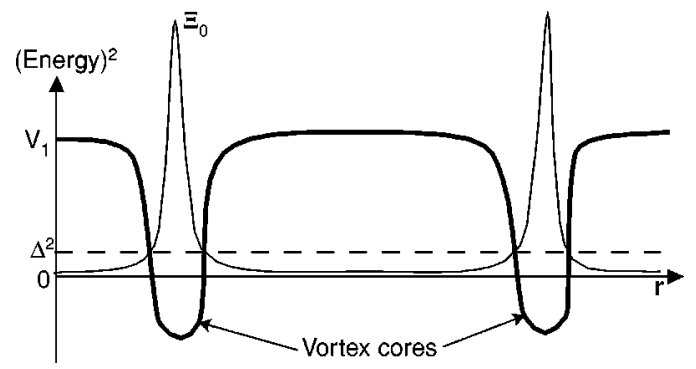

FIG. 1. A sketch of the potential $\mathcal{V}_{0}(\mathbf{r})$ (thick full line) in the presence of a vortex lattice. Also shown is the exciton wave function $\Xi_{0}(\mathbf{r})$ which solves Eq. (1.17) for $\mathcal{V}(\mathbf{r})=\mathcal{V}_{0}(\mathbf{r})$ with eigenvalue $\Delta^{2}$. Note that there is no drastic change in this picture as $\Delta^{2} \searrow 0$ : the peaks in $\Xi_{0}(\mathbf{r})$ remain exponentially localized within each vortex core, on a length scale much smaller than the vortex lattice spacing. We argue in the text that strong interaction corrections to $\mathcal{V}_{0}(\mathbf{r})$ invalidate this form for $\Xi_{0}(\mathbf{r})$ and the correct structure is shown in Fig. 2.

$$
\left[-v_{1}^{2} \partial_{x}^{2}-v_{2}^{2} \partial_{y}^{2}+\mathcal{V}(\mathbf{r})\right] \Xi_{0}(\mathbf{r})=\Delta^{2} \Xi_{0}(\mathbf{r}),
$$

where $\Xi_{0}(\mathbf{r})$ is the lowest eigenmode of Eq. (1.17), the eigenvalue $\Delta^{2}$ is required to be positive for the stability of the Gaussian theory $\mathcal{S}_{G}$. The energy $\Delta$ is the spin-gap, and $\Xi_{0}(\mathbf{r})$ then specifies the envelope of the lowest energy SDW fluctuations; in other words $\Xi_{0}(\mathbf{r})$ is the wave function of a $S=1$ exciton associated with dynamic SDW fluctuations. Note that $\Delta^{2}$ can be positive even if there are regions where $\mathcal{V}(\mathbf{r})<0$. A sketch of the spatial form of $\Xi_{0}(\mathbf{r})$ is shown in Fig. 1 for a particular small value of $\Delta^{2}$ and $\mathcal{V}(\mathbf{r})=\mathcal{V}_{0}(\mathbf{r})$. Observe that $\Xi_{0}(\mathbf{r})$ is peaked at the vortex centers, but decays rapidly outside the vortex cores over a SDW localization length $l \sim v_{1,2} / \sqrt{V_{1}-\Delta^{2}}$, where $V_{1}$ is the value of $\mathcal{V}_{0}(\mathbf{r})$ outside the vortex cores (see Fig. 1).

Remaining within the Gaussian theory specified by Eqs. (1.15) and (1.16), we now consider the consequences of raising the value of $H$ in the hope of reaching the SC+SDW phase. With increasing $H$, the vortex cores will approach each other, and we expect that the value of $\Delta^{2}$ will decrease. Indeed, the picture of Fig. 1 holds all the way up to the point $\Delta=0$; beyond this field the Gaussian theory becomes unstable and this signals the onset of the SC+SDW phase driven by the condensation of $\Phi_{x \alpha}$. Note that the localization length $l \sim v_{1,2} / \sqrt{V_{1}-\Delta^{2}}$ of the SDW order peaked in the vortex cores remains finite all the way up to the critical point. This localization length $l$ must be clearly distinguished from the spin correlation length $\xi_{s}$ : the latter is associated with correlations between different vortices, and arises because there is an exponentially small coupling between magnetism in neighboring cores. Thus this simple Gaussian theory yields a picture of dynamic magnetism appearing first in the vortex cores, with possible weak correlations between neighboring cores. Such a viewpoint was also discussed by Lake et al. ${ }^{12}$ who proposed "spins in the vortices" but noted that the large value of $\xi_{s}$ implied coupling between nearby vortices. Following our work, ${ }^{9} \mathrm{Hu}$ and Zhang $^{37}$ also presented a picture of dynamic SDW fluctuations similar to the one above. 
We now argue that corrections beyond the Gaussian theory approximation invalidate the above picture when $\Delta$ becomes small. ${ }^{9}$ Indeed, the picture of nearly independent, localized magnetic excitations in each vortex core holds only then $\Delta$ is of order the spin exchange energy $J$; such high energy magnetic excitations are expected to strongly damped by the fermionic quasiparticles. Also, the validity of the present continuum model is questionable at scales as short as vortex core size and at energies of order $J$ : a full solution of the BCS theory of the underlying electrons is surely needed, and subsidiary order parameters may well develop within the vortex cores. However, as $\Delta$ is lowered, we will now argue that the physics is actually dominated by the large region outside the vortex cores, where the present continuum approach can be used without fear, and the subtle issues of the short-distance physics within the core can be sidestepped. The central weakness in the analysis of the previous paragraph is that it does not account for the repulsive interactions $u_{1,2}$ between the bosonic $\Phi_{x \alpha}$ exciton modes that are condensing. As has been discussed in different contexts long ago, ${ }^{38,39}$ such interactions are crucial in determining the structure of the lowest-energy state in which condensation occurs. In particular, it is well known that the effect of interactions is to delocalize the lowest-energy states: bosons initially prefer to occupy strongly localized, low-energy states, but then their repulsive interaction with subsequent bosons drives the energy of such states up. Bray and Moore ${ }^{39}$ presented an argument demonstrating that in the vicinity of the condensation, the localization length must diverge as one approached the bottom of the band of states of interacting bosons in the presence of an external potential. To apply their argument in the present context, we need to replace Eq. (1.16) by

$$
\begin{aligned}
\mathcal{V}(\mathbf{r}) & =\mathcal{V}_{0}(\mathbf{r})+\frac{\left(4 u_{1}+2 u_{2}\right)}{3}\left\langle\left|\Phi_{x \alpha}(\mathbf{r}, \tau)\right|^{2}\right\rangle_{\mathcal{S}_{G}} \\
& =s+\kappa|\psi(\mathbf{r})|^{2}+\frac{\left(4 u_{1}+2 u_{2}\right)}{3}\left\langle\left|\Phi_{x \alpha}(\mathbf{r}, \tau)\right|^{2}\right\rangle_{\mathcal{S}_{G}}
\end{aligned}
$$

the additional terms arise from a Hartree-Fock decoupling of the quartic interaction terms in $\mathcal{S}_{\Phi}$, and the expectation values have to be evaluated self-consistently under the Gaussian action in Eq. (1.15) which itself depends upon $\mathcal{V}(\mathbf{r})$. Note that the perspective of magnetic criticality requires that we account for the $u_{1,2}$ interactions, as these are strongly relevant perturbations about the Gaussian theory; so we are led to Eq. (1.18) also by a naive application of the RG approach. We will present detailed numerical solutions of equations closely related to Eq. (1.18) in the body of the paper. An adaption of the argument of Bray and Moore ${ }^{39}$ to Eq. (1.18) was given in Ref. 9, and we will not repeat it here: the main result is that the length scale $l$ characterizing the lowestenergy state $\Xi_{0}(\mathbf{r})$ cannot remain finite as $\Delta \searrow 0$. Instead the states around neighboring vortex cores overlap strongly, and $\Xi_{0}(\mathbf{r})$ is characterized by the vortex spacing itself. A sketch of the actual structure of $\Xi_{0}(\mathbf{r})$ is shown in Fig. 2. The spin correlation length $\xi_{s}$ does not have a direct connection with

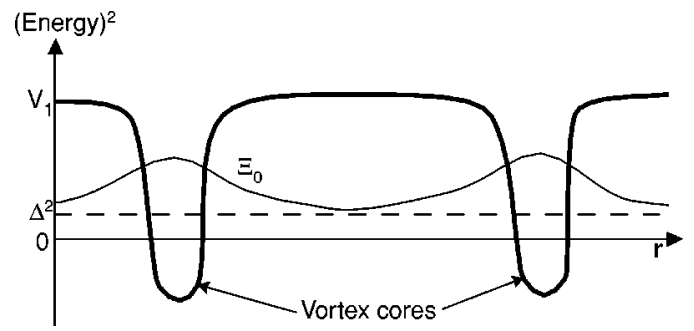

FIG. 2. A sketch of the potential $\mathcal{V}_{0}(\mathbf{r})$ (thick full line) in the presence of a vortex lattice along with the true form of the exciton wave function $\Xi_{0}(\mathbf{r})$ which solves Eq. (1.17) with the full potential $\mathcal{V}(\mathbf{r})$ in Eq. (1.18). The spatial structure of $\Xi_{0}(\mathbf{r})$ as $\Delta^{2} \searrow 0$ is characterized by the vortex lattice spacing.

the spatial form of $\Xi_{0}(\mathbf{r})$ itself, but is instead related to an integral over a band of states which solve Eq. (1.17) at finite momentum, as we shall discuss in Sec. I C 1 and later in the paper.

It is worth noting here that the passage from Eq. (1.16) to (1.18) in zero field is precisely that needed to reproduce the known properties of magnetic quantum critical points in other situations. In one dimension, Eq. (1.16) would imply that there is no barrier to magnetic long-range order, while Eq. (1.18) correctly implies that the presence of the Haldane gap, and reproduces its magnitude in the semiclassical limit. ${ }^{7}$ At finite temperature, Eq. (1.18) yields the correct crossovers in the magnetic correlation length in the vicinity of the spin ordering transition in two dimensions. Although we will not present detailed solutions on this case here, Eq. (1.18) is also expected to provide a reasonable description of the magnetic crossovers at finite temperatures in the vicinity of the SC + SDW to SC transition in the presence of a magnetic field.

With the knowledge of the spatial structure of the exciton wave function $\Xi_{0}(\mathbf{r})$ in Fig. 2, the origin of our main results ${ }^{9}$ can be easily understood. As the vortex cores occupy only a small fraction of the system volume, the magnitude of the energy $\Delta^{2}$ is influenced mainly by the structure of $\psi(\mathbf{r})$ in the remaining space. Here, the predominant consequence of the magnetic field is the presence of a superflow with velocity $\mathbf{v}_{s}=-\delta \mathcal{F} / \delta \mathbf{A}$ circulating around each vortex core. Focusing on the region around a single vortex at the origin $\mathbf{r}$ $=(0,0)$, the superflow obeys $\left|\mathbf{v}_{s}\right| \sim 1 / r$ in the wide region $\xi_{0}<r<L_{v}$ where $\xi_{0}=1 / \sqrt{2 m^{*} \alpha}$ is the vortex core size, $L_{v}$ $\sim\left(e^{*} H / c\right)^{-1 / 2}$; so the average superflow kinetic energy is

$$
\left\langle\mathbf{v}_{s}^{2}\right\rangle \propto \frac{\int_{\xi_{0}}^{L_{v}} \frac{d^{2} r}{r^{2}}}{\int_{\xi_{0}}^{L_{v}} d^{2} r} \propto \frac{H}{H_{c 2}^{0}} \ln \left(\frac{H_{c 2}^{0}}{H}\right),
$$

where $H_{c 2}^{0}$ is the upper critical field for the destruction of the Meissner state at the coupling constant corresponding to the point $\mathrm{M}$ in Fig. 3 below. This kinetic energy is a scalar with the same quantum numbers and symmetry properties as $|\psi|^{2}$ : hence, via the coupling in $\mathcal{S}_{\Phi \psi}$ in Eq. (1.10), the value of Eq. 


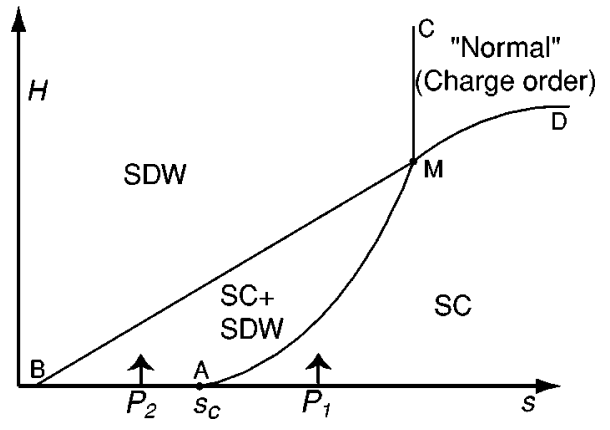

FIG. 3. Zero temperature phase diagram as a function of the coupling $s$ and the magnetic field $H$ in an extreme type-II superconductor described by Eq. (1.13). The theory is accurate in the region of small $H$, and only qualitatively correct elsewhere $[H$ is measured in units described in Eq. (3.2)]. The phases without SC order are likely to be insulators, and the "normal" phase is expected to have residual CDW order, which is initially induced by the pinning terms in $\mathcal{S}_{\text {lat }}$ as discussed in Sec. I C 1 . The positions of the phase boundaries are summarized in Sec. III. The path $P_{1}$ denotes the location of the original neutron-scattering measurements of Lake et al. (Ref. 12 ), and the path $P_{2}$ the subsequent neutron scattering measurements of Khaykovich et al. (Ref. 13) and Lake et al. (Ref. 14). The STM measurements of Hoffman et al. (Ref. 18) are also along path $P_{1}$.

(1.19) feeds into all the effective coupling constants in $\mathcal{S}_{\Phi}$ in Eq. (1.8). The most important modification is that the tuning parameter $s$ gets replaced by

$$
s_{\text {eff }}(H)=s-\mathcal{C} \frac{H}{H_{c 2}^{0}} \ln \left(\frac{H_{c 2}^{0}}{H}\right),
$$

where $\mathcal{C}$ is a constant of order unity. The implication of Eq. (1.20) is that we may as well replace $\mathcal{V}(\mathbf{r})$ in Eqs. (1.15) and (1.18) by

$$
\mathcal{V}(\mathbf{r}) \approx s_{\text {eff }}(H)
$$

to obtain a first estimate of the consequence of the magnetic field in the vicinity of the SC+SDW to SC transition. The $H$ dependence in Eqs. (1.20) and (1.21) is sufficient to determine our main results: ${ }^{9}$ the small $H$ portion of the phase diagram in Fig. 3, the intensity of the elastic scattering Bragg peak in the SC+SDW phase, and the energy of the lowestenergy SDW fluctuation in the SC phase. In particular, it follows directly from Eq. (1.20) that the small $H$ portion of the AM phase boundary in Fig. 3 between the SC and SC + SDW phases behaves as

$$
H \sim \frac{2\left(s-s_{c}\right)}{\kappa \ln \left[1 /\left(s-s_{c}\right)\right]} .
$$

Note that this phase boundary approaches the $s=s_{c}, H=0$ quantum critical point with vanishing slope. This implies that a relatively small $H$ for $s>s_{c}$ will successfully move the system close the AM phase boundary, and so produce low energy spin excitations. This should be contrasted to the corresponding $H$-dependent phase boundary of the SDW phase in insulators which is discussed in Appendix B; here, there is no orbital diamagnetism, only the Zeeman coupling is operative, and the phase boundary approaches $H=0$ with infinite slope. Evidently, Zeeman effects are much weaker and can be justifiably neglected.

We conclude this subsection by a brief discussion of earlier works ${ }^{32-34,40-50}$ on vortex magnetism, and the change in perspective that has been offered here by our analysis. It was proposed in by Sachdev ${ }^{40}$ and Nagaosa and Lee ${ }^{41}$ that vortex cores in the underdoped cuprates should have spin gap correlations characteristic of Mott insulators. Zhang ${ }^{32}$ and Arovas et al. ${ }^{33}$ described vortex core correlations in terms of static Néel order, and estimated that the field-induced moment would be proportional to $H$ [in our phase diagram in Fig. 3, vortices with static moments are only present in the $\mathrm{SC}+\mathrm{SDW}$ phase, and as we will review below in Sec. V D, the average moment increases as ${ }^{9} H \ln (1 / H)$ for small $\left.H\right]$. Our discussion here also uses the SDW order parameter, but allows it to fluctuate dynamically into a spin gap state, and so interpolates between these earlier works. A separate description of vortex cores in terms of "staggered flux" correlations ${ }^{49,50}$ has also been proposed. One of our central points here is that while the vortex core correlations may well be quite complicated (they are dependent on lattice scale effects, and difficult to distinguish from each other as the short-distance "order" fluctuates dynamically), these issues can be sidestepped: a reliable continuum theory can be developed by considering first the dominant effects arising from the interplay between superconductivity and magnetism in the superflow region outside the vortex cores. Spindensity-wave correlations induced in these regions may leak into the vortex cores, but our treatment is not expected to be reliable in the latter region: the nature of the electronic correlations in the vortex cores remains an open question.

Our continuum treatment of dynamic and static spindensity-wave order differs from earlier works in several key aspects. An important feature of Refs. 33,34 is the static mean-field treatment of the SDW order in the vortex cores, which is imposed by their " $\mathrm{SO}(5)$ " picture of SC order outside the cores "rotating" into static antiferromagnetism in the cores. ${ }^{36}$ This should be contrasted to our approach, in which magnetic quantum criticality implies dynamic magnetic fluctuations while the SC order can be safely considered static. Further, Refs. 33,34 assumed the (near) equality of the gradient and "mass" terms for the SC and twosublattice SDW order parameters, as naturally suggested by the dynamic $\mathrm{SO}(5)$ symmetry, which requires a symmetry between the excited states in the SC and SDW phases. As a result they found static two-sublattice magnetization induced by the vortex core, over a scale which was of order the vortex core size $\xi_{0}$ and in a regime where superconductivity was essentially completely suppressed. This assumption was relaxed in a recent paper, ${ }^{37}$ where following our work, ${ }^{9}$ the possibility of a generalized dynamic SDW in regions larger than the nonsuperconducting core, and coexisting with wellestablished superconductivity, was appreciated. $\mathrm{Hu}$ and Zhang $^{37}$ also suggested that a small proximity-type coupling between the magnetic domains centered on the neighboring vortices may be sufficient to stabilize static long-range magnetic order in a SC+SDW phase, in which enhancement of 
the SDW in the vortex cores was the dominant effect. As we have reviewed at length above, the strongly relevant exciton self-interactions lead to a different description of the SC + SDW phase ${ }^{9}$ in the SC phase, as one approaches the SC + SDW phase, the SDW order is induced over large length scales outside the vortex core, and the influence of the superflow is paramount. Only in the regime where magnetic field is small and the system is well within the SC phase (and far from the SC to SC+SDW boundary), can we speak in terms of localized bound state pulled below the continuum. However, this limit is of little experimental interest, since it corresponds to high energy magnetic excitons (which, as discussed above, are probably strongly overdamped by other excitations associated with the vortex cores) with a vanishingly small intensity.

\section{Pinning of charge order in the SC phase.}

Our physical discussion has so far neglected the influence of the pinning potential in $\mathcal{S}_{\text {lat }}$ in Eq. (1.12). We will continue to neglect this term in most of this paper, apart from computations in Sec. IV E whose content we briefly describe here. This analysis is motivated by the STM experiments of Hoffman et al. ${ }^{18}$

The SC phase of Fig. 3 preserves spin rotation invariance, and so has $\left\langle\Phi_{x \alpha}\right\rangle=0$ and, by Eq. (1.2), $\left\langle S_{\alpha}\right\rangle=0$ [if we were to account for the small Zeeman term (Appendix B), the analogous statement holds for the spin density in the plane perpendicular to the magnetic field]. In the absence of $\mathcal{S}_{\text {lat }}$, all the remaining terms in the partition function $\mathcal{Z}$ in Eq. (1.13) are invariant under the sliding symmetry $\Phi_{x \alpha}$ $\rightarrow e^{i \theta} \Phi_{x \alpha}$, and so we also have $\left\langle\Phi_{x \alpha}^{2}\right\rangle=0$ and, by Eqs. $(1.5),(1.6),\langle\delta \rho\rangle=0$ in the SC phase. Now if we include the effect of $\mathcal{S}_{\text {lat }}$ perturbatively (which is all we shall do here), the pinning of the dynamic fluctuations by the vortex cores leads to static CDW order with $\left\langle\Phi_{x \alpha}^{2}\right\rangle \neq 0$ and $\langle\delta \rho\rangle \neq 0$, while the continued preservation of spin rotation invariance implies that we still have $\left\langle\Phi_{x \alpha}\right\rangle=0$ and $\left\langle S_{\alpha}\right\rangle=0$. (Of course in the other SC+SDW phase, spin rotation symmetry is broken, and so $\left\langle\Phi_{x \alpha}\right\rangle \neq 0$ and $\left\langle S_{\alpha}\right\rangle \neq 0$, along with static CDW order.)

The nucleation of static CDW order, but with dynamic SDW order, in the SC phase by the vortices was first predicted in Refs. 10,51, where a connection was also made with lattice scale studies of bond-centered charge order correlations in superconductors with preserved spin rotation invariance. ${ }^{26}$ These latter works found a significant doping range over which the charge order had a period pinned at four lattice spacings, which is the period observed in the STM experiments of Hoffman et al. ${ }^{18}$ (the same period also appeared in density matrix renormalization group studies by White and Scalapino ${ }^{52}$ ). Here we are interested in the spatial extent of the envelope of the period four charge order. Following Ref. 11, here we will compute this envelope using our present models for dynamic SDW/CDW fluctuations in the SC phase, and the pinning of a static CDW by $\mathcal{S}_{\text {lat }}$.

After this paper was originally released, we learned of the microscopic model of Chen and Ting ${ }^{53}$ for the STM experiments, which follows the earlier work of Ref. 48. Their model has static order for both the SDW and CDW, and thus would apply only in the SC+SDW phase of our phase diagram in Fig. 3. It appears unlikely to us that the slightly overdoped BSCCO sample used by Hoffman et al. ${ }^{18}$ is in the $\mathrm{SC}+\mathrm{SDW}$ phase.

The simple model of the field-induced dynamic SDW fluctuations we have described in this section can be readily extended to compute the static CDW order induced by $\zeta$ in the SC phase. Indeed, the upshot of our preceeding discussion of the extended structure of $\Xi_{0}(\mathbf{r})$ is that we can use the Gaussian theory $\mathcal{S}_{G}$ in Eq. (1.15) with $\mathcal{V}(\mathbf{r})$ given by the constant value in Eq. (1.21): computing $\left\langle\Phi_{x \alpha}^{2}\right\rangle$ in the theory $\mathcal{S}_{G}+\mathcal{S}_{\text {lat }}$ for this value of $\mathcal{V}(\mathbf{r})$ and to first order in $\zeta$, we find $^{11}$

$$
\begin{aligned}
\left\langle\Phi_{x \alpha}^{2}(\mathbf{r}, \tau)\right\rangle= & \sum_{\mathbf{r}_{v}}\left(\frac{3}{8 \pi^{3 / 2}\left[s_{\mathrm{eff}}(H)\right]^{1 / 4} v^{5 / 2}}\right) \\
& \times \zeta e^{-i \varpi} \frac{e^{-2\left|\mathbf{r}-\mathbf{r}_{v}\right| \sqrt{s_{\mathrm{eff}}(H)} / v}}{\left|\mathbf{r}-\mathbf{r}_{v}\right|^{3 / 2}},
\end{aligned}
$$

where $\left|\mathbf{r}-\mathbf{r}_{v}\right| \equiv v\left\{\left[\left(x-x_{v}\right) / v_{1}\right]^{2}+\left[\left(y-y_{v}\right) / v_{2}\right]^{2}\right\}^{1 / 2}$ and $v$ $=\left(v_{1} v_{2}\right)^{1 / 2}$; the result (1.23) holds for large $\left|\mathbf{r}-\mathbf{r}_{v}\right|$, and the divergence at small $\left|\mathbf{r}-\mathbf{r}_{v}\right|$ it cutoff by lattice scale effects. Note that the static CDW order decays exponentially around each vortex core over a length scale $\xi_{c}=v /\left[2 \sqrt{s_{\text {eff }}(H)}\right]$ which has been increased by the influence of the fieldinduced superflow [by the decrease of $s_{\text {eff }}(H)$ in Eq. (1.20)]. Note also that this length scale is not related to any localization scale associated with the SDW state $\Xi_{0}(\mathbf{r})$; indeed, we have argued above that the latter state is extended. In the present simple Gaussian calculation, we used the very simple constant potential given in Eq. (1.21) in the Schrödinger equation for the exciton, Eq. (1.17); all eigenstates of such an equation are extended plane-wave states. Instead, the exponential decay in Eq. (1.23) arises from the integral over all the oscillating (but extended) excited states of Eq. (1.17). The body of the paper will show that the same feature also holds when the full form of $\mathcal{V}(\mathbf{r})$ is used, and not just the crude approximation in Eq. (1.21) (see Figs. 15 and 16).

It is useful to make an analogy between the above result and the phenomenon of Friedel oscillations in a Fermi liquid. A Fermi liquid state has no static SDW or CDW order, but there are enhanced fluctuations of these orders at $2 k_{F}$, the wave vector which spans extremal points of the Fermi surface. In the presence of an external impurity, static CDW oscillations at $2 k_{F}$ are induced, while full spin-rotation invariance is preserved. The amplitude of these oscillations decay with a power-law because the Fermi liquid has gapless spectrum of SDW/CDW excitations.

In the present situation, the physics of the doped Mott insulator induces a preference for excitonic SDW fluctuations at the wave vectors $\mathbf{K}_{s x, y}$ and for CDW fluctuations at the wave vectors $\mathbf{K}_{c x, y}=2 \mathbf{K}_{s x, y}$. The $\mathrm{SC}$ phase has a spin gap $\Delta$ at these wave vectors, and so such spin correlations decay exponentially on the scale $\xi_{s}=v / \Delta$ (as we have noted, this is not a localization scale of the spin exciton states, which are all extended). The vortex core pins the phase the 
dynamic SDW fluctuations which reside above this spin gap, and the resulting "Friedel oscillations of the spin gap" are manifested by static CDW oscillations at the wave vectors $\mathbf{K}_{c x, y}$ whose envelope decays exponentially over a length scale $\xi_{c}=\xi_{s} / 2$. These may therefore be viewed as the Friedel oscillations associated with the excitonic bound states that are present below the particle-hole continuum of the $d$-wave superconductor. In a weak-coupling BCS/RPA theory one can also expect additional Friedel oscillations associated with the continuum of particle-hole excitations, whose wave vector is determined by the quasiparticle dispersion. Such a picture may be appropriate in the strongly overdoped limit in zero magnetic field, with pinning provided by impurities. However, as one lowers the doping in the SC phase (to approach the boundary to the SC+SDW phase), an excitonic bound state appears, and we have focused on its physics here; the wave vector of this exciton is determined by strongcoupling effects in the doped Mott insulator. The strength of this exciton could also be enhanced relative to the particlehole continuum in the vicinity of vortices in an applied magnetic field-this effect requires explicit consideration of the fermionic quasiparticles, and so is beyond the scope of the theories considered here.

The outline of the remainder of this paper is as follows. We will begin in Sec. II by a discussion of the phase diagram of the spin and charge-density-wave order parameters in zero magnetic field. More complex phases and phase diagrams are also possible, associated the composites and "fractions" of these order parameters, but we will postpone their discussion until Sec. VI. We will turn to the influence of the magnetic field in Sec. III: here we will restrict our attention to the quantum transition described by $\mathcal{S}_{\Phi}$, but most of the zerofield transitions discussed in Sec. VI have a related response to an applied magnetic field. Sec. III contains a description of the phase diagram in the magnetic field, while the subsequent sections describe the dynamic and static properties of the two phases on either side of the critical point in some detail: Sec. IV describes the SC phase, while Sec. V describes the SC+SDW phase. Section VII reviews earlier theoretical and experimental work on the interplay of magnetism and superconductivity, and discuss its relationship to our treatment here. We conclude in Sec. VIII by considering implications of our results for recent experiments; readers not interested in theoretical details may skip ahead to Sec. VIII now. A number of technical and numerical details appear in the appendixes.

\section{PHASE DIAGRAM IN ZERO MAGNETIC FIELD}

We orient ourselves by discussing the phase diagrams of models with various types of spin- and charge-density-wave order. We will restrict our attention in this section to zero external field, assume that a background SC order is always present in all the phases. As we have argued above, this implies that we need not consider the SC order parameter explicitly, and its influence only serves to renormalize various couplings in the effective actions. A somewhat different viewpoint, with a more explicit role for the SC order, has been taken recently by Lee. ${ }^{54}$

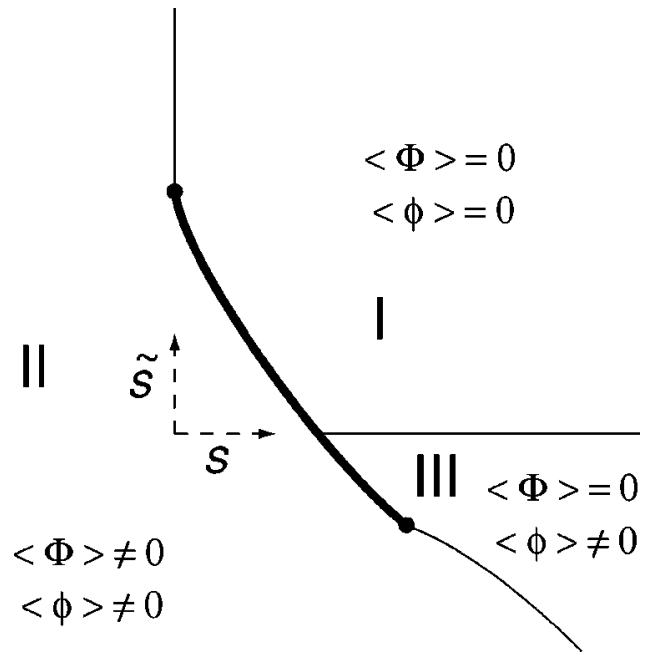

FIG. 4. Mean-field, zero-temperature phase diagram of the zero magnetic field model $\mathcal{S}_{\Phi}+\mathcal{S}_{\phi}+\mathcal{S}_{\Phi \phi}$ defined in Eqs. (1.8),(2.1),(2.2), with $u_{2}<0$.

Here, we consider phases that are characterized simply by the condensates of one or more of the order parameters $\Phi_{x, y \alpha}$ and $\phi_{x, y}$, introduced in Sec. I A. More complex phases associated with composites or "fractions" of these fields are also possible and these will be considered later in Sec. VI. However, the remainder of the paper will only deal with the influence of the magnetic field on phases and phase boundaries associated with the order parameters $\Phi_{x, y \alpha}$ and $\phi_{x, y}$; the more complex cases have similar properties which can be described in an analogous manner.

To characterize the simple phases we need an effective action $\mathcal{S}_{\phi}$ for the $\phi_{x, y}$, while that for $\Phi_{x, y \alpha}$ is $\mathcal{S}_{\Phi}$ in Eq. (1.8); the former can be written down using a reasoning similar to that for Eq. (1.8), and we obtain

$$
\begin{aligned}
\mathcal{S}_{\phi}= & \int d^{2} r d \tau\left[\left|\partial_{\tau} \phi_{x}\right|^{2}+\tilde{v}_{1}^{2}\left|\partial_{x} \phi_{x}\right|^{2}+\tilde{v}_{2}^{2}\left|\partial_{y} \phi_{x}\right|^{2}+\left|\partial_{\tau} \phi_{y}\right|^{2}\right. \\
& +\tilde{v}_{1}^{2}\left|\partial_{x} \phi_{y}\right|^{2}+\tilde{v}_{2}^{2}\left|\partial_{y} \phi_{y}\right|^{2}+\tilde{s}\left(\left|\phi_{x}\right|^{2}+\left|\phi_{y}\right|^{2}\right)+\frac{\tilde{u}_{1}}{2}\left(\left|\phi_{x}\right|^{4}\right. \\
& \left.\left.+\left|\phi_{y}\right|^{4}\right)+\tilde{w}_{1}\left|\phi_{x}\right|^{2}\left|\phi_{y}\right|^{2}\right] .
\end{aligned}
$$

The correspondence (1.6) implies that for $\mathbf{K}_{c x}=2 \mathbf{K}_{s x}$ and $\mathbf{K}_{c y}=2 \mathbf{K}_{s y}$ the SDW and CDW order parameters are coupled by

$$
\mathcal{S}_{\Phi \phi}=-\lambda \int d^{2} r d \tau\left[\phi_{x}^{*} \Phi_{x \alpha}^{2}+\phi_{y}^{*} \Phi_{y \alpha}^{2}+\text { c.c. }\right]
$$

without loss of generality, we can assume that the coupling $\lambda>0$. At the mean-field level, the properties of the quantum model $\mathcal{S}_{\Phi}+\mathcal{S}_{\phi}+\mathcal{S}_{\Phi \phi}$ are essentially identical to the classical models considered by Zachar et al. ${ }^{23}$ for spin and chargeordering transitions at nonzero temperature; so we can directly borrow their results, and a characteristic mean-field phase diagram is shown in Fig. 4. 
Next, we discuss the critical properties of the various second-order quantum transitions in Fig. 4. Near the transition between phase II (SC+SDW) and the symmetric phase I (SC), the primary order parameters are $\Phi_{x, y \alpha}$. We can integrate out the noncritical $\phi_{x, y}$ fields and this merely renormalizes the couplings in $\mathcal{S}_{\Phi}$. So the theory $\mathcal{S}_{\Phi}$ is the critical theory for this transition at $H=0$. This is a model of some complexity, and the universal critical properties of related simpler models are the focus of some debate in the literature, ${ }^{55-59}$ these earlier results are briefly reviewed in Appendix D. These previous studies correspond to the case where $\Phi_{x \alpha}$ and $\Phi_{y \alpha}$ are decoupled $\left(w_{1}=w_{2}=w_{3}=0\right)$, and weakly first-order transitions are obtained in some cases. We will address the generalization of these previous analyses to the case of nonzero $w_{1,2,3}$ in future work. Here, we will be satisfied by considering the simplest, and most symmetric, case of a second-order transition: for the special values $v_{1}$ $=v_{2}, u_{1}=w_{1}, u_{2}=w_{2}=w_{3}=0$ the model $\mathcal{S}_{\Phi}$ has $\mathrm{O}(12)$ symmetry, and its properties are identical to that of the $(N$ =12)-component $\varphi^{4}$ theory $\mathcal{S}_{\varphi}$ to be described shortly below. The influence of $H$ on other second-order or weakly first-order transitions should be very similar, with the changes only modifying the numerical values of certain asymptotic critical parameters. Part of our reason for not expending much effort on this point is that these asymptotic critical are not particularly relevant for the experimental situation in $H \neq 0$ anyway: after including the small effects of $\mathcal{S}_{\text {lat }}$ in Eq. (1.12), the "sliding" symmetry of $\mathcal{S}_{\Phi}$ disappears, and the asymptotic critical properties of the SC+SDW to SC transition in $H \neq 0$ become identical to the $(N=3)$ component $\varphi^{4}$ theory $\mathcal{S}_{\varphi}$. We will discuss the $H \neq 0$ properties of $\mathcal{S}_{\varphi}$ at some length in this paper, and we expect that closely related results apply to the generalized $\mathcal{S}_{\Phi}$ and to $\mathcal{S}_{\Phi}+\mathcal{S}_{\text {lat }}$.

Near the transition between phases III and I in Fig. 4, the roles of $\Phi_{x, y \alpha}$ and $\phi_{x, y}$ are reversed. Now we can integrate out the noncritical $\Phi_{x, y \alpha}$, this renormalizes the couplings in $\mathcal{S}_{\phi}$, and the renormalized $\mathcal{S}_{\Phi}$ is the critical theory for this transition at $H=0$. At nonzero $H$, a model closely related to the one discussed above applies. We will not explicitly present the results for this model here, as most physical properties are essentially identical to those of $\mathcal{S}_{\Phi}+\mathcal{S}_{\psi}+\mathcal{S}_{\Phi \psi}$.

The remaining second order quantum transition in Fig. 4 is that between phases II and III. Both these phases have $\left\langle\phi_{x, y}\right\rangle \neq 0$, and the charge order can be viewed as a noncritical spectator to the transition. For specificity, let us assume that $\left\langle\phi_{x}\right\rangle$ is real and positive, while $\left\langle\phi_{y}\right\rangle=0$; other cases lead to similar final results. Now replace $\phi_{x, y}$ by their expectation values in $\mathcal{S}_{\Phi}+\mathcal{S}_{\Phi \phi}$ in Eqs. (1.8),(2.2), and examine fluctuations of $\Phi_{x, y \alpha}$ at the Gaussian level: those of $\operatorname{Re}\left[\Phi_{x \alpha}\right]$ have an energy lower than all other components. Close to phase boundary between II and III we can therefore assume that the critical theory involves only $\varphi_{\alpha}(\mathbf{r}, \tau)$ $\equiv \operatorname{Re}\left[\Phi_{x \alpha}(\mathbf{r}, \tau)\right]$, and all other components only renormalize the couplings in its effective action. In this manner, we can conclude that the II to III phase transition is described by the familiar $(N=3)$-component $\varphi^{4}$ field theory, with effective action

$$
\begin{aligned}
\mathcal{S}_{\varphi}= & \int d^{2} r d \tau\left\{\frac { 1 } { 2 } \left[\left(\partial_{\tau} \varphi_{\alpha}\right)^{2}+v^{2}\left(\nabla_{\mathbf{r}} \varphi_{\alpha}\right)^{2}\right.\right. \\
& \left.\left.+\left[s+\kappa|\psi(\mathbf{r})|^{2}\right] \varphi_{\alpha}^{2}\right]+\frac{u}{2}\left(\varphi_{\alpha}^{2}\right)^{2}\right\},
\end{aligned}
$$

where the index $\alpha=1 \cdots N$, and the field $\varphi_{\alpha}(\mathbf{r}, \tau)$ is real. We have rescaled spatial co-ordinates to make the velocities $v_{1,2}$ equal to the common value $v$. For completeness, we have also included the coupling to the SC order $\psi$ which derives from Eq. (1.10). An analysis of the properties of the theory $\mathcal{F} / T+\mathcal{S}_{\varphi}$, defined in Eqs. (1.9) and (2.3), in nonzero field shall occupy us in most of the remainder of the paper. Recall also that the $N=12$ case of this theory also describes a particular case of the I to II transition discussed earlier.

\section{PHASE DIAGRAM IN A MAGNETIC FIELD}

We now embark on a presentation of the main new results of this paper: a description of the phase diagram and the dynamic spin spectra of $\mathcal{F} / T+\mathcal{S}_{\varphi}$, defined in Eqs. (1.9) and (2.3), as a function of the applied field $H$. As discussed near Eq. (2.3), this theory describes the response of a number of specific phase boundaries of states with SDW/CDW order to an applied magnetic field; the number of components of $\varphi_{\alpha}$ takes the values $N=3,12$ depending upon the transition of interest, but we expect similar results for all values of $N$ $\geqslant 3$. Actually, closely related analyses can be applied to most of the phases to be discussed in Sec. VI. The basic effect, that all couplings associated with the non-superconducting order parameter acquire a $H \ln (1 / H)$ depends, is very robust and leads to analogous phase diagrams in almost all cases.

The theory $\mathcal{F} / T+\mathcal{S}_{\varphi}$ has a rather number of coupling constants, and it is useful to use our freedom to rescale lengths, times, and field scales to obtain an irreducible set of parameters whose values control the structure of our results. First, as is conventional in the standard Ginzburg-Landau theory of superconductivity, we introduce the superconducting coherence length $\xi_{0}$ and the field scales $H_{c}$ and $H_{c 2}^{0}$ :

$$
\begin{aligned}
& \xi_{0}=\sqrt{\frac{1}{2 m^{*} \alpha}}, \\
& H_{c}=\sqrt{\frac{4 \pi \alpha^{2}}{\beta}}, \\
& H_{c 2}^{0}=\frac{2 m^{*} \alpha c}{e^{*}},
\end{aligned}
$$

as we noted earlier, $H_{c 2}^{0}$ is the value of the upper critical field at the point M in Fig. 3 , and $H_{c 2}^{0}=\sqrt{2} \kappa H_{c}$, where $\kappa$ is usual the Ginzburg Landau parameter. We will also see below in Sec. IV B that the coupling $\alpha$ acquires a shift renormalization due to its coupling to $\varphi_{\alpha}$ fluctuations: we assume that renormalization has already been performed in the definitions (3.1). We now use the length $\xi_{0}$, the velocity $v$, and the 
parameters in Eq. (3.1) to set various length, time, temperature, field, and coupling constant scales; we define the dimensionless parameters

$$
\begin{gathered}
\tilde{\mathbf{r}}=\frac{\mathbf{r}}{\xi_{0}}, \quad \tilde{\tau}=\frac{v \tau}{\xi_{0}}, \quad \widetilde{T}=\frac{\xi_{0} T}{v}, \\
\widetilde{H}=\frac{H}{H_{c 2}^{0}}, \quad \tilde{\psi}=\sqrt{\frac{\beta}{\alpha}} \psi, \quad \tilde{\varphi}_{\alpha}=\sqrt{v \xi_{0}} \varphi_{\alpha}, \\
\tilde{s}=\frac{\xi_{0}^{2}}{v^{2}} s, \quad \tilde{u}=\frac{\xi_{0}}{v^{3}} u, \quad \tilde{\kappa}=\frac{\xi_{0}^{2} \alpha}{v^{2} \beta} \kappa .
\end{gathered}
$$

It is evident from the above that we are measuring length scales in units of $\xi_{0}$ and energy scales in units of $v / \xi_{0}$.

Collecting all the transformations, let us restate the problem we are going to solve; we drop all the tildes, and it is henceforth assumed that all parameters have been modified as in Eq. (3.2). The partition function in Eq. (1.13) is now simplified to

$$
\mathcal{Z}[\psi(\mathbf{r})]=\int \mathcal{D} \varphi_{\alpha}(\mathbf{r}, \tau) \exp \left(-\frac{\mathcal{F}}{T}-\mathcal{S}_{\varphi}\right),
$$

where $\mathcal{S}_{\varphi}$ is as in Eq. (2.3) but with $v=1$, while $\mathcal{F}$ is now given by

$$
\mathcal{F}=\Upsilon \int d^{2} r\left[-|\psi|^{2}+\frac{1}{2}|\psi|^{4}+\left|\left(\nabla_{\mathbf{r}}-i \mathbf{A}\right) \psi\right|^{2}\right] .
$$

The dimensionless constant $Y$ is given by

$$
\Upsilon=\frac{H_{c}^{2} \xi_{0}^{3} d}{4 \pi v}
$$

where $d$ is the interlayer spacing (this factor of $d$ is needed to make $\mathcal{Y}$ dimensionless, and arises because $\mathcal{F}$ is the free energy per layer); in determining $Y$, a useful unit of conversion is $1(\mathrm{~T})^{2}=0.0624 \mathrm{meV} \AA^{-3}$. The vector potential $\mathbf{A}$ in Eq. (3.4) now satisfies

$$
\boldsymbol{\nabla}_{\mathbf{r}} \times \mathbf{A}=H \hat{z}
$$

An important property of the continuum theory (3.3) is that all dependence on the short distance cutoff can be removed by a single "mass renormalization:" this amounts to measuring the tuning parameter $s$ in terms of its deviation from $s=s_{c}$, the critical point between the SC+SDW and SC phases at $H=0$. Consequently all physical properties are functions only of the dimensionless parameters $u, \kappa, \mathrm{Y}$, $H / H_{c 2}^{0}$, and $s-s_{c}$. We will present numerical results for the frequency and spatial dependence of various observables below as a function of $H / H_{c 2}^{0}$ and $s-s_{c}$ for the simple set of values $u=\kappa=\Upsilon=1$; we do not expect any qualitative changes for other values of these last three parameters. Also, it will occasionally be convenient to exchange the parameter $s-s_{c}$ for $\Delta$, the value of the spin gap in the $s>s_{c}$ SC phase at $H=0$.
The technical tool we shall use in our analysis of Eq. (3.3) is the large $N$ expansion. This approach ${ }^{35}$ is known to yield an accurate description of the vicinity of spin ordering quantum critical points in two dimensions, and we expect the same to hold here in the presence of a nonzero $H$. Details of the approach will emerge in the following sections: here we summarize the main $N=\infty$ results for the positions of the phase boundaries appearing in Fig. 3 .

The tetracritical point $M$ where all four phases meet is at $H=1, s-s_{c}=\kappa$.

The line BM represents the upper-critical field for the vanishing of superconductivity in the presence of SDW order; it is at

$$
H=1-\frac{\kappa^{2}}{4 u \Upsilon}+\frac{\kappa}{4 u \Upsilon}\left(s-s_{c}\right) .
$$

The line CM, the boundary for SDW order in the insulator, is at $s-s_{c}=\kappa$.

The line DM, the upper-critical field for superconductivity in the absence of SDW order is at

$$
H=1+\frac{N \kappa}{8 \pi \Upsilon}\left[\left(\frac{N^{2} u^{2}}{16 \pi^{2}}-\kappa+s-s_{c}\right)^{1 / 2}-\frac{N u}{4 \pi}\right] .
$$

Experimentally, the most important and accessible phase boundary is AM, the line representing onset of SDW order in the SC phase. The position of this line cannot be determined analytically: we will present detailed numerical results and an expansion in the vicinity of $M$; for small $H$ its location behaves as

$$
H \sim \frac{2\left(s-s_{c}\right)}{\kappa \ln \left[1 /\left(s-s_{c}\right)\right]},
$$

as may be readily deduced from Eq. (1.20), and was quoted already in Eq. (1.22).

Our numerical as well as analytical studies will be divided into two parts, one for "SC" region of Fig. 3 in Sec. IV, and the other for "SC+SDW" region in Sec. V.

\section{PHYSICAL PROPERTIES OF THE SC PHASE}

This section will describe an analysis of Eq. (3.3) in the regime where spin rotation invariance is preserved with $\left\langle\varphi_{\alpha}\right\rangle=0$. As we discussed earlier at the end of Sec. I C, upon including the effect of the lattice pinning term (1.12) in a nonzero $H$, this phase does have static CDW order with $\left\langle\phi_{x, y}\right\rangle \neq 0$, while preserving spin rotation invariance: this will be discussed in Sec. IV E.

\section{A. Large $N$ saddle point equations}

The index $\alpha$ in $\mathcal{S}_{\varphi}$ in Eq. (2.3) extends over $\alpha$ $=1, \ldots, N$, and depending upon the transition in Fig. 4 we are interested in, we have either $N=3$ or $N=12$. For both cases, it is known that an accurate description of the physical properties is described by the large $N$ expansion, whose implementation we shall now describe.

First, we introduce an auxiliary field 


$$
\mathcal{V}(\mathbf{r}, \tau)=s+\kappa\left|\psi_{H}(\mathbf{r})\right|^{2}+2 u \varphi_{\alpha}^{2}(\mathbf{r}, \tau) .
$$

We will often place a subscript $H$ on various quantities (as for $\psi$ above) to emphasize that they are being evaluated at a nonzero $H$. Let us also denote

$$
s^{\prime}=s+\kappa\left|\psi_{H}(\mathbf{r})\right|^{2} .
$$

Now we add an innocuous term to $\mathcal{S}_{\varphi}$, whose only effect is to multiply the partition function by a constant after a functional integration over $\mathcal{V}(\mathbf{r}, \tau)$ :

$$
\begin{aligned}
\mathcal{S}_{\varphi} \rightarrow & \mathcal{S}_{\varphi}-\int d^{2} r \int_{0}^{1 / T} d \tau \frac{1}{8 u}\left(\mathcal{V}-2 u \varphi_{\alpha}^{2}-s^{\prime}\right)^{2} \\
= & \int d^{2} r \int_{0}^{1 / T} d \tau\left[\frac{1}{2}\left(\partial_{\tau} \varphi_{\alpha}\right)^{2}+\frac{1}{2}\left(\nabla_{\mathbf{r}} \varphi_{\alpha}\right)^{2}\right. \\
& \left.-\frac{1}{8 u} \mathcal{V}^{2}+\frac{1}{2} \mathcal{V} \varphi_{\alpha}^{2}+\frac{1}{4 u} \mathcal{V}_{s^{\prime}}\right] .
\end{aligned}
$$

After integrating out $\varphi_{\alpha}(\alpha=1 \cdots N)$, we have

$$
\begin{aligned}
\mathcal{Z}= & \int \mathcal{D} \mathcal{V}(\mathbf{r}) \exp \left[-\frac{N}{2} \operatorname{Tr} \ln \left(-\partial_{\tau}^{2}-\nabla_{\mathbf{r}}^{2}+\mathcal{V}\right)\right. \\
& \left.-\frac{1}{4 u} \mathcal{V} s^{\prime}+\frac{1}{8 u} \mathcal{V}^{2}\right] .
\end{aligned}
$$

Now by taking $N \rightarrow \infty$ while keeping $N u$ constant, we obtain the saddle point equation in which $\mathcal{V}$ is a function of $\mathbf{r}$ but independent of $\tau$ :

$$
\mathcal{V}_{H}(\mathbf{r})=s+\kappa\left|\psi_{H}(\mathbf{r})\right|^{2}+2 N u T \sum_{\omega_{n}} G_{H}\left(\mathbf{r}, \mathbf{r}, \omega_{n}\right) .
$$

where the $\varphi_{\alpha}$ propagator $G_{H}\left(\mathbf{r}, \mathbf{r}^{\prime}, \omega_{n}\right)$ is given by

$$
G_{H}\left(\mathbf{r}, \mathbf{r}^{\prime}, \omega_{n}\right)=\left\langle\mathbf{r}\left|\left[\omega_{n}^{2}-\nabla_{\mathbf{r}}^{2}+\mathcal{V}_{H}(\mathbf{r})\right]^{-1}\right| \mathbf{r}^{\prime}\right\rangle,
$$

with $\omega_{n}$ a Matsubara frequency. In this case, the large- $N$ expansion is equivalent to a self-consistent one-loop calculation.

The saddle point equation for superconducting order parameter follows from Eq. (1.14): it is just the conventional Ginzburg-Landau equation with one additional term from the $\varphi, \psi$ coupling

$$
\begin{aligned}
& {\left[-1+\frac{N \kappa}{2 \Upsilon} T \sum_{\omega_{n}} G_{H}\left(\mathbf{r}, \mathbf{r}, \omega_{n}\right)+\left|\psi_{H}(\mathbf{r})\right|^{2}-\left(\boldsymbol{\nabla}_{\mathbf{r}}-i \mathbf{A}\right)^{2}\right] \psi_{H}(\mathbf{r})} \\
& \quad=0 .
\end{aligned}
$$

So the two unknown functions $\mathcal{V}_{H}(\mathbf{r})$ and $\psi_{H}(\mathbf{r})$ are to be determined simultaneously by the solution of Eqs. (4.5) and (4.7). As stated above, the expressions in these equations depend upon the short distance cutoff, but we show in Sec. IV $B$ that this can easily be removed by a simple shift of parameters.

\section{B. Renormalization of parameters}

It is first useful to obtain the complete solution of Eqs. (4.5) and (4.7) at $H=0$. Let $s=s_{c}$ be the point where magnetic order appears (so that $\left\langle\varphi_{\alpha}\right\rangle \neq 0$ for $s<s_{c}$ ), where ${ }^{35} \mathcal{V}$ $=0$. Then Eq. (4.5) tells us that

$$
0=s_{c}+\kappa\left|\psi_{0 c}\right|^{2}+2 N u \int \frac{d \omega}{2 \pi} \int \frac{d^{2} k}{4 \pi^{2}} \frac{1}{\omega^{2}+k^{2}},
$$

where $\psi_{0 c}$ is the $\mathbf{r}$ independent value of $\psi(\mathbf{r})$ at $s=s_{c}$ and $H=0$, while Eq. (4.7) gives

$$
-1+\left|\psi_{0 c}\right|^{2}+\frac{N \kappa}{2 \Upsilon} \int \frac{d \omega}{2 \pi} \int \frac{d^{2} k}{4 \pi^{2}} \frac{1}{\omega^{2}+k^{2}}=0 .
$$

It is useful to normalize things so that $\psi_{0 c}=1$ at $s=s_{c}, H$ $=0$ and $T=0$. This is achieved if we renormalize $\alpha$ to remove the offending term in Eq. (4.9). We make the shift in Eq. (1.9) [before the rescalings in Eq. (3.2)]

$$
\alpha \rightarrow \alpha+\frac{N \kappa \beta}{2 \alpha} \int \frac{d \omega}{2 \pi} \int \frac{d^{2} k}{4 \pi^{2}} \frac{1}{\omega^{2}+v^{2} k^{2}} .
$$

Then, after Eq. (3.2), Eq. (4.7) is modified to

$$
\begin{gathered}
\left\{-1+\frac{N \kappa}{2 \Upsilon}\left[T \sum_{\omega_{n}} G_{H}\left(\mathbf{r}, \mathbf{r}, \omega_{n}\right)-\int \frac{d \omega d^{2} k}{8 \pi^{3}} \frac{1}{\omega^{2}+k^{2}}\right]\right. \\
\left.+\left|\psi_{H}(\mathbf{r})\right|^{2}-\left(\nabla_{\mathbf{r}}-i \mathbf{A}\right)^{2}\right\} \psi_{H}(\mathbf{r})=0
\end{gathered}
$$

while Eq. (4.9) simply becomes

$$
\psi_{0 c}=1
$$

Now move to $s>s_{c}$, where we have a spin gap

$$
\Delta_{0} \equiv \sqrt{\mathcal{V}_{0}}>0
$$

Subtracting Eq. (4.8) from Eq. (4.5) we get

$$
\Delta_{0}^{2}=s-s_{c}+\kappa\left(\left|\psi_{0}\right|^{2}-1\right)-\frac{N u \Delta_{0}}{2 \pi}
$$

where Eq. (4.11) yields

$$
\left|\psi_{0}\right|^{2}=1+\frac{N \kappa \Delta_{0}}{8 \pi \Upsilon} .
$$

Inserting Eq. (4.15) back into Eq. (4.14) we obtain

$$
\Delta_{0}^{2}+\frac{N u}{2 \pi}\left(1-\frac{\kappa^{2}}{4 u \Upsilon}\right) \Delta_{0}=s-s_{c} .
$$

Let us now use the above equations to simplify the equations for $H \neq 0$ and $T \neq 0$. The new form will be independent of lattice cutoff.

From Eqs. (4.5),(4.8),(4.16) we obtain 


$$
\begin{aligned}
\mathcal{V}_{H}(\mathbf{r})= & \Delta_{0}^{2}+\kappa\left[\left|\psi_{H}(\mathbf{r})\right|^{2}-\left|\psi_{0}\right|^{2}\right]+2 N u\left[T \sum_{\omega_{n}} G_{H}\left(\mathbf{r}, \mathbf{r}, \omega_{n}\right)\right. \\
& \left.-\int \frac{d \omega}{2 \pi} \frac{d^{2} k}{4 \pi^{2}} \frac{1}{\omega^{2}+k^{2}+\Delta_{0}^{2}}\right]
\end{aligned}
$$

where $\left|\psi_{0}\right|^{2}$ is given in Eq. (4.15). Using Eqs. (4.17) and (4.11) we obtain

$$
\begin{aligned}
& {\left[\left(1-\frac{\kappa^{2}}{4 u \Upsilon}\right)\left[\left|\psi_{H}(\mathbf{r})\right|^{2}-\left|\psi_{0}\right|^{2}\right]\right.} \\
& \left.\quad+\frac{\kappa}{4 u \Upsilon}\left[\mathcal{V}_{H}(\mathbf{r})-\Delta_{0}^{2}\right]-\left(\nabla_{\mathbf{r}}-i \mathbf{A}\right)^{2}\right] \psi_{H}(\mathbf{r})=0 .
\end{aligned}
$$

The expressions (4.17),(4.18) are the main equations we shall solve for the unknowns $\mathcal{V}_{H}(\mathbf{r})$ and $\psi_{H}(\mathbf{r})$ in this paper. It can be checked that at $H=0, T=0$, these equations are solved by $\mathcal{V}_{H}=\Delta_{0}^{2}$ and $\psi_{H}=\psi_{0}$. We describe the numerical solution of these equations for $H \neq 0$ in Appendix $\mathrm{E}$ and present the results in the following subsection. A useful step in this numerical solution is the following parametrization of the Green's function $G_{H}\left(\mathbf{r}, \mathbf{r}^{\prime}, \omega_{n}\right)$ in Eq. (4.6)

$$
G_{H}\left(\mathbf{r}, \mathbf{r}^{\prime}, \omega_{n}\right)=\sum_{\mu} \int_{1 B Z 4 \pi^{2}} \frac{d^{2} k}{\omega_{n}^{*}+E_{\mu}^{2}(\mathbf{k})},
$$

where $\Xi_{\mu \mathbf{k}}(\mathbf{r})$ are the complete set of eigenfunctions of the analog of the Schrödinger equation (1.17)

$$
\left[-\nabla_{\mathbf{r}}^{2}+\mathcal{V}_{H}(\mathbf{r})\right] \Xi_{\mu \mathbf{k}}(\mathbf{r})=E_{\mu}^{2}(\mathbf{k}) \Xi_{\mu \mathbf{k}}(\mathbf{r})
$$

Here $\mathbf{k}$ is a "Bloch" momentum which extends over the first Brillouin zone of the vortex lattice, $\mu$ is a "band" index, and $E_{\mu}(\mathbf{k})$ are the corresponding energy eigenvalues. All of our numerical analysis was performed for the values $u=\kappa=\Upsilon$ $=1$ and $N=3$.

\section{Phase boundaries}

Equations (4.17) and (4.18) can be readily solved to obtain the locations of the CM and DM phase boundaries in Fig. 3. On DM, the superconducting phase parameter $\psi_{H}(\mathbf{r})$ vanishes and all parameters become $\mathbf{r}$ independent; thus Eq. (4.17) becomes

$$
\mathcal{V}_{H}=\Delta_{0}^{2}-\kappa\left|\psi_{0}\right|^{2}+\frac{N u}{2 \pi}\left(\sqrt{\mathcal{V}_{H}}-\Delta_{0}\right)=s-s_{c}-\kappa-\frac{N u}{2 \pi} \sqrt{\mathcal{V}_{H}}
$$

where we used Eqs. (4.15) and (4.14). Then from Eq. (4.18) we have

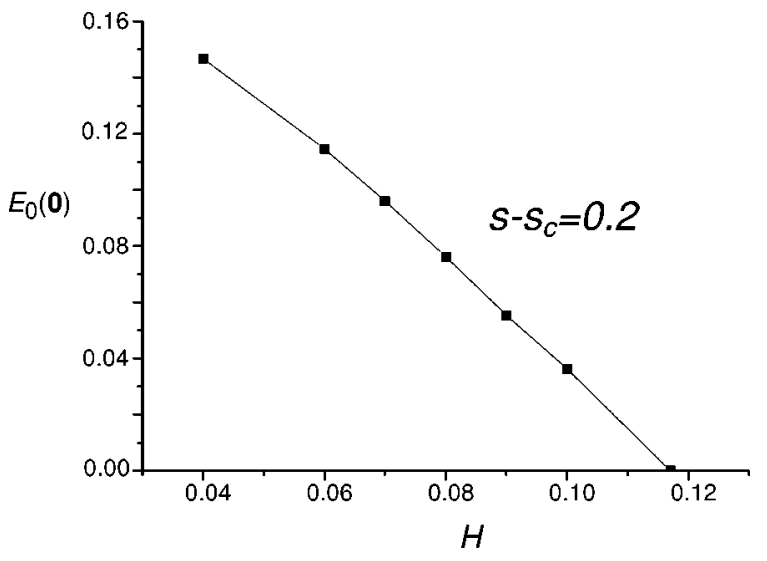

FIG. 5. The lowest eigenvalue of Eq. (1.17), $E_{0}(\mathbf{0})$ vs $H$ for $s$ $-s_{c}=0.2$. The linear continuation of the line to solve $E_{0}(\mathbf{0})=0$ gives us the critical $H$ for this $s$, which is about 0.117 with an uncertainty of \pm 0.002 .

$$
\begin{aligned}
H & =1-\frac{\kappa^{2}}{4 u \Upsilon}+\frac{\kappa}{4 u \Upsilon}\left(s-s_{c}-\mathcal{V}_{H}\right) \\
& =1-\frac{\kappa^{2}}{4 u \Upsilon}+\frac{\kappa}{4 u \Upsilon}\left(\frac{N u}{2 \pi} \sqrt{\mathcal{V}_{H}}+\kappa\right) \\
& =1+\frac{N \kappa}{8 \pi \Upsilon}\left[\left(\frac{N^{2} u^{2}}{16 \pi^{2}}-\kappa+s-s_{c}\right)^{1 / 2}-\frac{N u}{4 \pi}\right],
\end{aligned}
$$

which is the result quoted in Eq. (3.8). Similarly, it is easy to see that the phase boundary $\mathrm{CM}$ is at $s-s_{c}=\kappa$.

It remains to determine the location of the phase boundary $\mathrm{AM}$, which is also physically the most interesting one. We determined this boundary by a full numerical solution of Eqs. (4.17) and (4.18) for a range of parameters. Stability of the SC phase requires that all the eigenvalues $E_{\mu}^{2}(\mathbf{k})$ of Eq. (1.17) remain positive. The lowest of these eigenvalues is $E_{0}(\mathbf{0})$ and we followed its behavior as a function $H$ : a typical result is shown in Fig. 5 . We expect $E_{0}(\mathbf{0})$ to vanish linearly in the deviation from the critical field, as the critical theory is expected to be in the universality class of the ordinary $\mathrm{O}(3)$ $\varphi^{4}$ field theory, and the latter has critical exponent $z \nu=1$ in the large $N$ limit. So we can determine the critical field by a linear extrapolation, and this is also shown in Fig. 5. Combining the results of such calculations at a range of values of $s$, we obtain our numerical result for the location of the AM boundary shown in Fig. 6.

Some further analytic results on the location of the AM phase boundary can be obtained in the vicinity of the multicritical point $M$. It can be shown that the deviation of the phase boundary from $\mathrm{M}$ is linear in the large $N$ limit, i.e., it is at $H=1-\varrho\left(\kappa-s+s_{c}\right)$, where $\varrho$ is a numerical constant. We describe these results in Appendix F, including the determination of $\varrho$. The results obtained in this manner are consistent with our complete numerical analysis described above, and this is a strong check on our numerical analysis.

Finally, we recall our result (3.9) for the behavior of AM at small $H$ and $s-s_{c}$. Here there is a crucial logarithm which follows from Eq. (1.20), and whose physical origin 


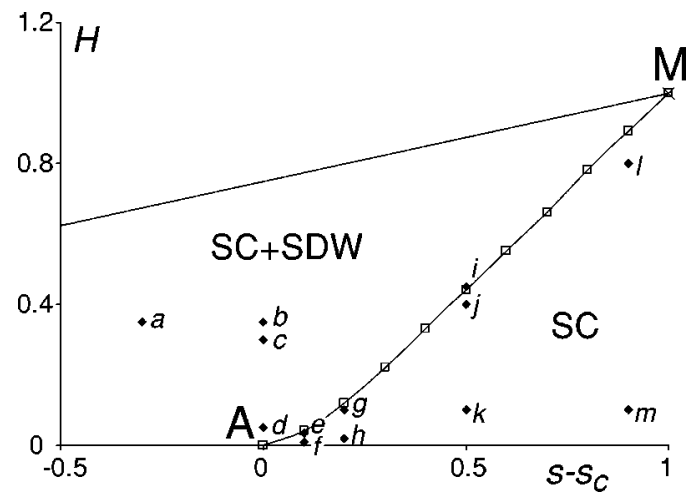

FIG. 6. Numerical results for the phase boundary AM in Fig. 3 for $u=\kappa=\Upsilon=1$. Also shown is a portion of the phase boundary $\mathrm{BM}$ whose position is known analytically from Eq. (3.7). Different aspects of the physical properties are described in the remainder of the paper at the points labeled $\mathrm{a}-1$.

was discussed in Sec. IC. The signal of this logarithm are clearly visible in the phase boundary in Fig. 6.

\section{Dynamic spin susceptibility}

In this section we describe the evolution of the dynamic spin fluctuation spectrum in the SC phase of Fig. 3. This is clearly specified by the Green's function $G_{H}\left(\mathbf{r}, \mathbf{r}^{\prime}, \omega_{n}\right)$ in Eq. (4.6) which we computed above in determining the phase boundary. More specifically, we see from Eq. (1.2) that the observed dynamic spin susceptibility $\chi(\mathbf{q}, \omega)$ is given by

$$
\begin{gathered}
\chi(\mathbf{q}, \omega) \propto \chi_{\varphi}\left(\mathbf{q}+\mathbf{K}_{s x}, \omega\right)+\chi_{\varphi}\left(\mathbf{q}-\mathbf{K}_{s x}, \omega\right) \\
+\chi_{\varphi}\left(\mathbf{q}+\mathbf{K}_{s y}, \omega\right)+\chi_{\varphi}\left(\mathbf{q}-\mathbf{K}_{s y}, \omega\right),
\end{gathered}
$$

where $\chi_{\varphi}$, the dynamic susceptibility for the field $\varphi_{\alpha}$, is given by

$$
\begin{aligned}
\chi_{\varphi}(\mathbf{k}, \omega) & =\frac{1}{V} \int d^{2} r d^{2} r^{\prime} e^{i \mathbf{k} \cdot\left(\mathbf{r}-\mathbf{r}^{\prime}\right)} G_{H}\left(\mathbf{r}, \mathbf{r}^{\prime}, \omega\right) \\
& =\sum_{\mu, \mathbf{G}} \int_{1 \mathrm{BZ}} d^{2} p \delta(\mathbf{p}+\mathbf{G}-\mathbf{k}) \frac{\left|c_{\mu \mathbf{G}}(\mathbf{p})\right|^{2}}{E_{\mu}^{2}(\mathbf{p})-\omega^{2}},
\end{aligned}
$$

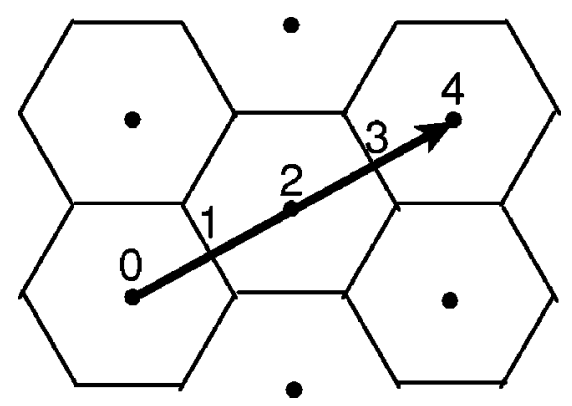

FIG. 7. Reciprocal lattice of the vortex lattice. The density plots in Figs. 8, 9, 10, and 11 have $\mathbf{k}$ varying along the arrow shown, with numerical values as shown.

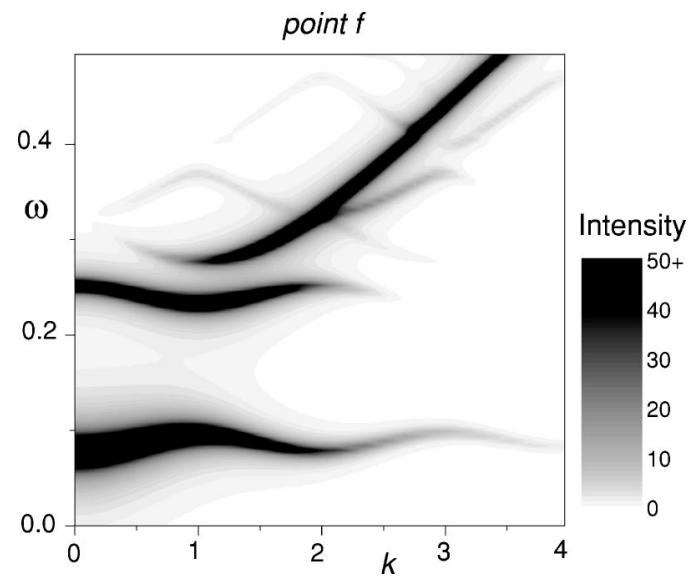

FIG. 8. Density plot of $\operatorname{Im} \chi_{\varphi}$ in Eq. (4.24) in the SC phase for momenta along the arrow in Fig. 7. The plot is for $s-s_{c}=0.1$ and $H=0.01$ (point $f$ in Fig. 6). In this, and all subsequent plots of $\operatorname{Im} \chi_{\varphi}$, the delta function peaks in (4.24) have been broadened into Lorentzians with energy width 0.01 for display purposes only.

where $V$ is the volume of the system, the $\mathbf{p}$ integration is over the first Brillouin zone of the reciprocal vortex lattice, $\mathbf{G}$ extends over the reciprocal lattice vectors of the vortex lattice, $E_{\mu}^{2}(\mathbf{p})$ are the eigenvalues of Eq. (4.20) (see also Appendix E), and the parameters $c_{\mu} \mathbf{G}(\mathbf{p})$ are defined in Eq. (E2). We present results for $\operatorname{Im}\left[\chi_{\varphi}(\mathbf{k}, \omega)\right]$ below.

It is clear from Eq. (4.24) that in the present large $N$ approximation, the spectrum of $\chi_{\varphi}$ consists entirely of sharp delta functions. These specify the dispersion of $S=1$ "excitons" which describe the SDW fluctuations, and are connected with the zero field "resonance" peak discussed early on in Sec. I. The excitons scatter off the vortex lattice, and our results describe the evolution of the resulting spectrum as one moves towards the onset of SDW order by increasing the applied magnetic field. We show the structure of $\operatorname{Im}\left[\chi_{\varphi}(\mathbf{k}, \omega)\right]$ by broadening the delta functions into sharp Lorentzians, and displaying the results in density plots. The momentum $\mathbf{k}$ in these plots varies along the direction of the reciprocal lattice shown in Fig. 7. The results for a smaller

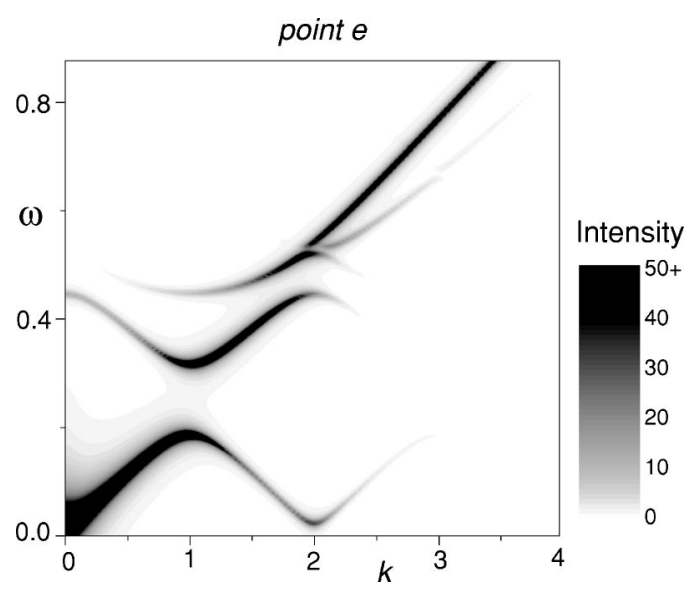

FIG. 9. As in Fig. 8 but for larger $H=0.035$, which brings the system very close to the AM phase boundary to the SC+SDW phase (point $e$ in Fig. 6). 


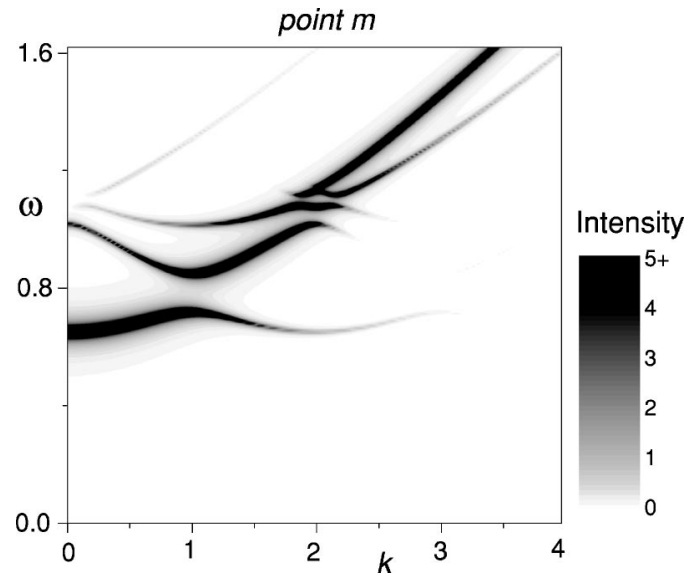

FIG. 10. As in Fig. 8 but with larger $s-s_{c}: H=0.1$ and $s-s_{c}$ $=0.9$ (point $m$ in Fig. 6).

value of $s-s_{c}$ are shown in Figs. 8 and 9, and those for larger value of $s-s_{c}$ are in Figs. 10 and 11. Note that for very small $H$, there is less dispersion for the lowest mode: this is an indication that this excitation is centered on the vortex core, and there is weaker coupling between neighboring vortices. As the field is increased, this coupling increases, and the dispersion looks closer to that of a nearly free particle, with weak reflections at the Brillouin zone boundaries of the vortex lattice. Also, the energy of the minimum excitation decreases with increasing field, until it vanishes at the AM phase boundary to the SC+SDW phase.

We also show in Figs. 12 and 13 the spatial structure of the modulus of the superconducting order parameter $\left|\psi_{H}(\mathbf{r})\right|^{2}$. The Brillouin zone boundary reflections above arise from the scattering of the exciton off the potential created by $\left|\psi_{H}(\mathbf{r})\right|^{2}$.

Finally, for experimental comparisons, it is useful to plot the intensity of the lowest exciton mode as a function of the applied field. From Eq. (4.24) we see that this intensity is $\left|c_{00}(\mathbf{0})\right|^{2}$. We show a plot of this quantity in Fig. 14. Observe that except for very small values of $H$, the intensity is of order unity, which is the behavior expected for an extended

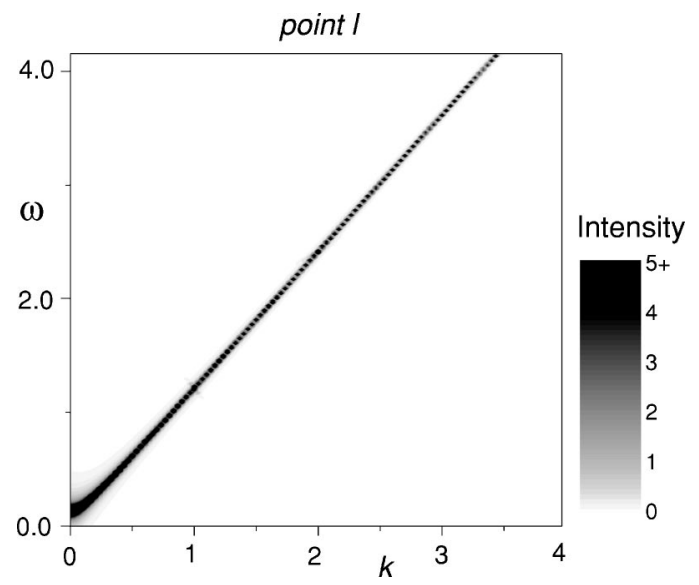

FIG. 11. As in Fig. 10 but with a large $H=0.8$, which brings the system very close to the AM phase boundary to the SC+SDW phase (point $l$ in Fig. 6).

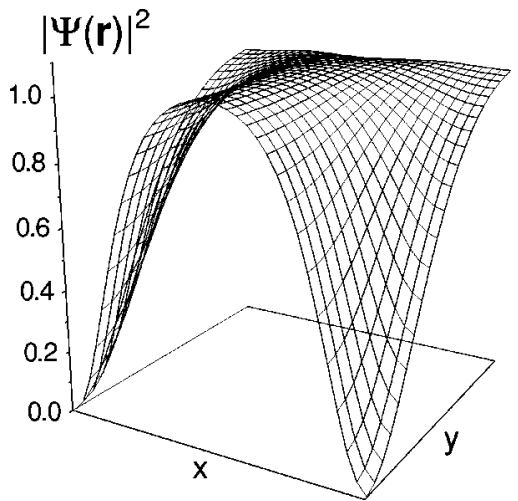

FIG. 12. Spatial dependence of the modulus of the superconducting order parameter $\left|\psi_{H}(\mathbf{r})\right|^{2}$ plotted on the rectangular half unit cell of the vortex lattice indicated by Fig. 13. This result is for $s-s_{c}=0.5$ and $H=0.1$ (point $k$ in Fig. 6).

exciton scattering off a periodic potential as in Fig. 12. As $H \rightarrow 0$, the behavior crosses over to that expected when the vortex cores are essentially decoupled, and the lowest mode is associated with a state localized around each vortex core: in this limit, we expect ${ }^{37}$ the intensity $\sim H$.

\section{E. Pinning of charge order}

This section will consider the consequences of the pinning term $\mathcal{S}_{\text {lat }}$ in Eq. (1.12). We argued at the end of Sec. I C that this term pins the charge order, and leads to a static CDW with $\left\langle\phi_{x, y}\right\rangle \neq 0$ [recall Eq. (1.5)] in the SC phase, while preserving spin rotation invariance with $\left\langle\Phi_{x, y \alpha}\right\rangle=0$. We have recently proposed ${ }^{11}$ this as an explanation for the CDW observed around the vortex in the STM measurements of Hoffman et al. ${ }^{18}$ Section I C, also gave an initial estimate [in Eq. (1.23)] of the spatial structure of this pinned CDW: here we will obtain a more precise result, using the full solution of the SDW fluctuations in the presence of the vortex lattice. Using the relationship (1.6) between the CDW and SDW orders in the vicinity of the SC to SC+SDW transition, we conclude that to first order in $\zeta$

$$
\left\langle\phi_{x, y}(\mathbf{r})\right\rangle \propto \zeta e^{-i \varpi} \Omega(\mathbf{r})
$$

with

$$
\Omega(\mathbf{r}) \equiv T \sum_{\omega_{n}} \sum_{\mathbf{r}_{v}} G_{H}^{2}\left(\mathbf{r}, \mathbf{r}_{v}, \omega_{n}\right),
$$

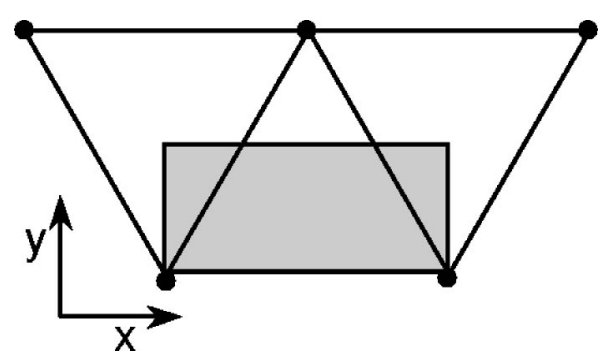

FIG. 13. Half unit cell of the triangular vortex lattice in real space. 


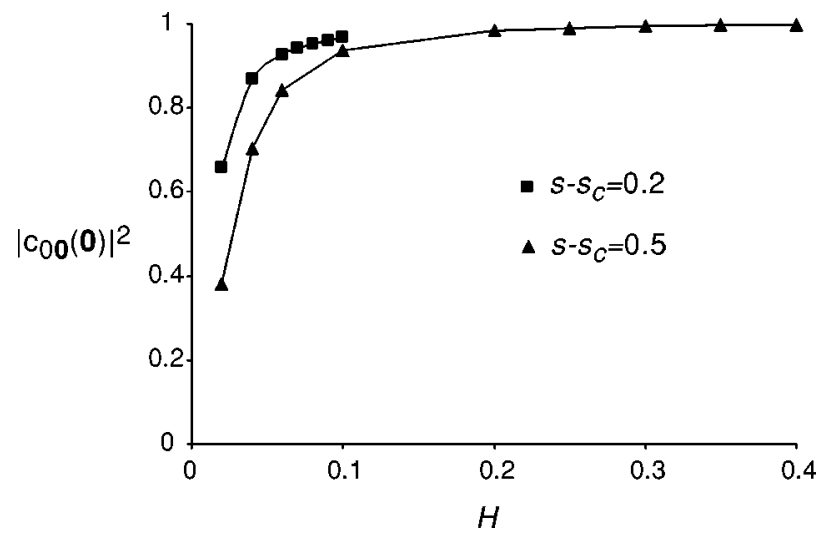

FIG. 14. Intensity of the lowest exciton mode in the SC phase $\left|c_{00}(\mathbf{0})\right|^{2}$ as a function of $H$ for two values of $s-s_{c}$.

where $\mathbf{r}_{v}$ extends over the vortex lattice sites; clearly $\Omega(\mathbf{r})$ has the full periodicity of the vortex lattice.

We used our numerical solution of Eqs. (4.17) and (4.18) to compute the function $\Omega(\mathbf{r})$, which is proportional to the amplitude of the static CDW induced by the vortex lattice in the spin gap phase. We show our results for $\Omega(\mathbf{r})$ in Figs. 15

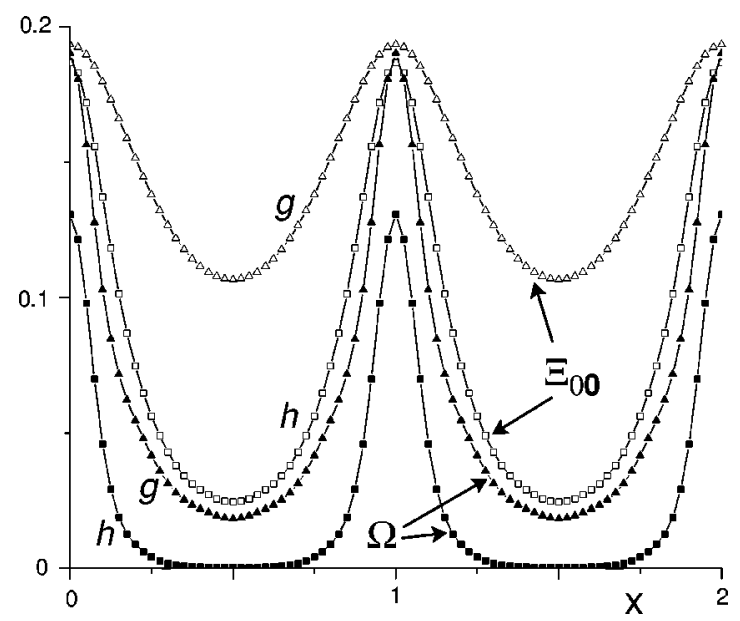

FIG. 15. Plots of the function $\Omega$ (r) (filled symbols) in Eq. (4.26) representing the static CDW order pinned by the vortices, along with the lowest SDW eigenfunction $\Xi_{00}(\mathbf{r})$ of the dynamic spin fluctuations above the spin gap (open symbols), at $s-s_{c}$ $=0.2$. The spatial coordinate $x$ is along the line connecting two nearest-neighbor vortices and its scale has been chosen so that the vortex lattice spacing is unity (see Fig. 13). The field takes the values $H=0.02$ (squares, point $h$ in Fig. 6) and $H=0.1$ (triangles, point $g$ in Fig. 6); the latter field is close to the AM phase boundary in Fig. 6. Note that the spin exciton state at point $g$ is well extended through the lattice, while the charge order remains localized around the vortices. For point $h$ the localization length of the spin exciton state is about twice that of the charge order. These results are consistent with the discussion in Sec. I C 1. As was also noted below Eq. (1.23), the continuum expression (4.26) actually has a divergence for $\mathbf{r}$ equal to any $\mathbf{r}_{v}$ : our numerical computation uses a finite momentum cutoff $\Lambda$, and this rounds out the divergence at distances $\left|\mathbf{r}-\mathbf{r}_{v}\right| \lesssim \Lambda^{-1}$; we have verified this by numerical computations at different $\Lambda$. In the same units as those for $x$ in the figure, we used $\Lambda \approx 36$ above.

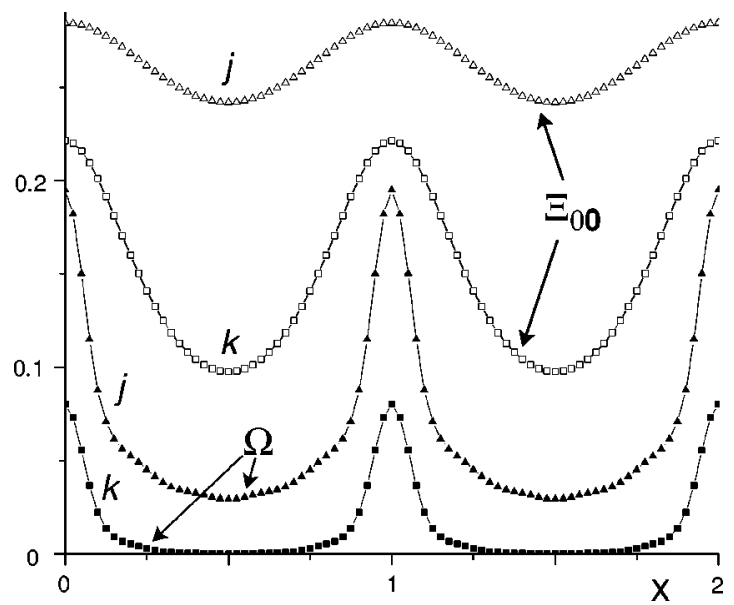

FIG. 16. As in Fig. 15 but for $s-s_{c}=0.5$. The field takes the values $H=0.1$ (squares, point $k$ in Fig. 6) and $H=0.4$ (triangles, point $j$ in Fig. 6); the latter field is close to the AM phase boundary in Fig. 6. Now both points have extended spin exciton states (that at point $j$ is essentially a plane wave), while the charge order is exponentially localized.

and 16 for a representative set of values in the SC phase. Also shown in the same figures, for orientation, is the form of $\Xi_{00}(\mathbf{r})$, the lowest-energy eigenfunction of the dynamic SDW equation (1.17) which appears in the Green's function (4.19). For very small field, both $\Omega(\mathbf{r})$ and $\Xi_{00}(\mathbf{r})$ are localized around the vortex centers, with the localization length of the former being about half that of the latter. However, for larger fields, the exciton wave function $\Xi_{00}(\mathbf{r})$ gets delocalized, while the CDW order remains localized. This localization arises from the summation over all the states in Eq. (4.19) and is in keeping with the discussion at the end of Sec. I C 1.

\section{PHYSICAL PROPERTIES OF THE SC+SDW PHASE}

We now turn to the analysis of the partition function (3.3) in the phase with broken spin rotation invariance and $\left\langle\varphi_{\alpha}\right\rangle$ $=0$. This phase is reached when the lowest $S=1$ exciton mode in Sec. IV, $\Xi_{00}(\mathbf{r})$, reaches zero energy $\left[E_{0}(\mathbf{0})=0\right]$ and then condenses. The presence of the condensate leads to long-range SDW order. We will adapt our large $N$ computation to include such a condensate in the following subsection, and then describe the spatial structure of the condensate and the dynamic spin excitations.

\section{A. Large- $N$ saddle point equations}

The analysis here is parallel to that in Sec. IV A. We introduce the auxiliary field $\mathcal{V}(\mathbf{r}, \tau)$ defined in Eq. (4.1) and write the action in the form similar to Eq. (4.3). However, to account for the condensate, we have to select a particular orientation in spin space, and treat the corresponding spin component in a selective manner. So we write

$$
\varphi_{\alpha}=\left(\sqrt{N} n, \pi_{1}, \pi_{2}, \ldots, \pi_{N-1}\right),
$$

and integrate out only $\pi_{1}, \pi_{2}, \ldots, \pi_{N-1}$ to obtain 


$$
\begin{aligned}
\mathcal{Z}= & \int \mathcal{D} \mathcal{V}(\mathbf{r}, \tau) \mathcal{D} n(\mathbf{r}, \tau) \exp \left[-\frac{N-1}{2} \operatorname{Tr} \ln \left(-\partial_{\tau}^{2}-\boldsymbol{\nabla}_{\mathbf{r}}^{2}+\mathcal{V}\right)\right. \\
& \left.+\frac{1}{8 u} \mathcal{V}^{2}-\frac{1}{4 u} \mathcal{V} s^{\prime}-\frac{N}{2}\left(\partial_{\tau} n\right)^{2}-\frac{N}{2}\left(\nabla_{\mathbf{r}} n\right)^{2}-\frac{N}{2} \mathcal{V} n^{2}\right],
\end{aligned}
$$

where $s^{\prime}$ was defined in Eq. (4.2). Now we take $N \rightarrow \infty$ while keeping $N u$ fixed, and ignoring the difference between $N$ and $N-1$. This leads to saddle point equations for the timeindependent field $\mathcal{V}_{H}(\mathbf{r})$ and the SDW condensate $n_{H}(\mathbf{r})$; these equations replace Eq. (4.5), but contain additional terms due to the spontaneous spin polarization

$$
\mathcal{V}_{H}(\mathbf{r})=s+\kappa\left|\psi_{H}(\mathbf{r})\right|^{2}+2 N u T \sum_{\omega_{n}} G_{H}\left(\mathbf{r}, \mathbf{r}, \omega_{n}\right)+2 N u n_{H}^{2}(\mathbf{r})
$$

and

$$
\left[-\nabla_{\mathbf{r}}^{2}+\mathcal{V}_{H}(\mathbf{r})\right] n_{H}(\mathbf{r})=0
$$

where $G_{H}$ is given by Eq. (4.6). Comparing Eqs. (5.4) and (4.6) it is easy to see that the spectrum of $G_{H}$, as defined in Eq. (4.19) has one mode with $E_{0}(\mathbf{k}) \rightarrow 0$ as $\mathbf{k} \rightarrow 0$; this is, of course, the Goldstone spin wave mode associated with the spontaneous SDW condensate.

The equation which determined the superconducting order parameter $\psi_{H}(\mathbf{r})$ was Eq. (4.7), and this is now replaced by

$$
\begin{gathered}
\left\{-1+\frac{N \kappa}{2 \Upsilon}\left[T \sum_{\omega_{n}} G_{H}\left(\mathbf{r}, \mathbf{r}, \omega_{n}\right)+2 N u n_{H}^{2}(\mathbf{r})\right]\right. \\
\left.+\left|\psi_{H}(\mathbf{r})\right|^{2}-\left(\nabla_{\mathbf{r}}-i \mathbf{A}\right)^{2}\right\} \psi_{H}(\mathbf{r})=0 .
\end{gathered}
$$

\section{B. Renormalization of parameters}

Now we proceed as in Sec. IV B to remove all dependence of Eqs. (5.3), (5.4), and (5.5) on the short-distance cutoff. First consider the case when $T=0, H=0$, and $s$ $=s_{c}$, where Eqs. (4.8) and (4.9) hold. Now after we shift parameter $\alpha$ in as in Eq. (4.10), the Ginzburg-Landau equation (5.5) is modified to

$$
\begin{gathered}
\left\{-1+\frac{N \kappa}{2 \Upsilon}\left[T \sum_{\omega_{n}} G_{H}\left(\mathbf{r}, \mathbf{r}, \omega_{n}\right)+2 N u n_{H}^{2}(\mathbf{r})\right.\right. \\
\left.-\int \frac{d \omega}{2 \pi} \int \frac{d^{2} k}{4 \pi^{2}} \frac{1}{\omega^{2}+k^{2}}\right]+\left|\psi_{H}(\mathbf{r})\right|^{2} \\
\left.-\left(\nabla_{\mathbf{r}}-i \mathbf{A}\right)^{2}\right\} \psi_{H}(\mathbf{r})=0 .
\end{gathered}
$$

Next, subtracting Eq. (4.8) from Eq. (5.3) while noticing that $\psi_{0 c}$ is already renormalized to unity, we have

$$
\begin{aligned}
\mathcal{V}_{H}(\mathbf{r})= & s-s_{c}+\kappa\left[\left|\psi_{H}(\mathbf{r})\right|^{2}-1\right]+2 N u n_{H}^{2}(\mathbf{r}) \\
& +2 N u\left[T \sum_{\omega_{n}} G_{H}\left(\mathbf{r}, \mathbf{r}, \omega_{n}\right)-\int \frac{d \omega d^{2} k}{8 \pi^{3}} \frac{1}{\omega^{2}+k^{2}}\right] .
\end{aligned}
$$

From Eqs. (5.7) and (5.6) we have

$$
\begin{aligned}
& {\left[\left(1-\frac{\kappa^{2}}{4 u \Upsilon}\right)\left[\left|\psi_{H}(\mathbf{r})\right|^{2}-1\right]+\frac{\kappa}{4 u \Upsilon}\left[\mathcal{V}_{H}(\mathbf{r})-s+s_{c}\right]\right.} \\
& \left.\quad-\left(\boldsymbol{\nabla}_{\mathbf{r}}-i \mathbf{A}\right)^{2}\right] \psi_{H}(\mathbf{r})=0
\end{aligned}
$$

The final set of equations for the properties of the SC + SDW phase are Eqs. (5.7), (5.4), and (5.8); these are to be solved for the unknowns $\mathcal{V}_{H}(\mathbf{r}), \psi_{H}(\mathbf{r})$, and $n_{H}(\mathbf{r})$. We describe the numerical solution in Appendix $\mathrm{G}$.

\section{Phase boundaries}

We have already determined the positions of several phase boundaries in Fig. 3 in Sec. IV C, and it remains only to determine BM. First notice that at the transition into a nonsuperconducting phase, the order parameter $\psi(\mathbf{r})$ vanishes, and thus $\mathcal{V}_{H}(\mathbf{r})$ and $\zeta_{H}(\mathbf{r})$ are spatially uniform. So from Eq. (5.4) we have $\mathcal{V}_{H}=0$. Plugging this into Eq. (5.6) we obtain the position of the phase boundary BM specified in Eq. (3.7).

\section{SDW order parameter}

The presence of a static spin condensate implies that the dynamic spin susceptibility contains sharp Bragg peaks at zero frequency and at wave vectors separated from the SDW ordering wave vectors by the reciprocal lattice vectors of the vortex lattice as suggested by Zhang ${ }^{32}$ and discussed by us in Ref. 9; these are in addition to the dynamic spectra specified in Eq. (4.24). This means that the dynamic structure factor $S_{\varphi}(\mathbf{k}, \omega)$ [which is related to the susceptibility $\chi_{\varphi}(\mathbf{k}, \omega)$ in Eq. (4.24) by the usual fluctuation-dissipation theorem] has the contributions

$$
S_{\varphi}(\mathbf{k}, \omega)=(2 \pi) \delta(\omega) \sum_{\mathbf{G}}\left|f_{\mathbf{G}}\right|^{2}(2 \pi)^{2} \delta(\mathbf{k}-\mathbf{G}),
$$

where $\mathbf{G}$ extends over the reciprocal lattice vectors of the vortex lattice, and

$$
f_{\mathbf{G}}=\frac{\sqrt{N}}{A_{\mathcal{U}}} \int_{\mathcal{U}} d^{2} r e^{-i \mathbf{G} \cdot \mathbf{r}_{n}} n_{H}(\mathbf{r}),
$$

where the spatial integral is over $\mathcal{U}$ the unit cell of the vortex lattice with area $A_{\mathcal{U}}$. Note that, by Eq. (4.23), the physical momentum is related to $\mathbf{k}$ in Eq. (5.9) by shifts from the SDW ordering wave vectors $\mathbf{K}_{s x}$ and $\mathbf{K}_{s y}$.

Figures 17 and 18 show plots of the Bragg scattering intensity $\left|f_{\mathbf{G}}\right|^{2}$, for the two smallest values of $\mathbf{G}$ and two values of $s-s_{c}$, as a function of $H$. As argued in Ref. 9, the corre- 


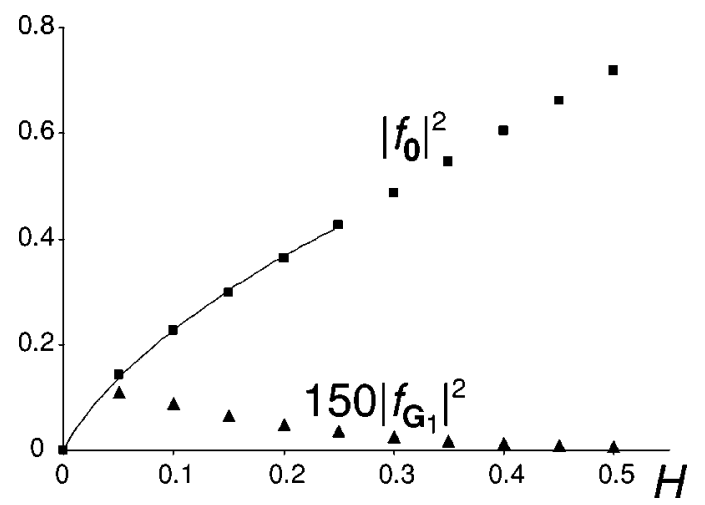

FIG. 17. Bragg scattering intensity $\left|f_{\mathbf{G}}\right|^{2}$ as a function of $H$ at $s-s_{c}=0$. Shown are the values at $\mathbf{G}=0$ (squares) and at $\mathbf{G}=\mathbf{G}_{1}$ (triangles), which is the smallest nonzero reciprocal lattice vector of the vortex lattice. Note that the intensities at $\mathbf{G}=\mathbf{G}_{1}$ have been magnified by a factor of 150 to make them visible on this plot. The intensities are zero at $H=0$, because $s=s_{c}$ is the quantum critical point in zero field. The line shows $0.63 H \ln (3.61 / H)$, which is the best fit to the functional form in Eq. (5.11).

spondence (1.20) implies that the scattering intensity at zero wave vector, $\left|f_{\mathbf{0}}\right|^{2}$ should increase with as

$$
\left\langle\left|f_{0}\right|^{2}\right\rangle \propto H \ln (1 / H) .
$$

The fits to this functional form in Fig. 17 show that this works quite well. Notice also that the intensity at the first nonzero reciprocal lattice vector $\mathbf{G}_{1}$ is quite small, and that it decreases with increasing $H$. This suggests that observation of this satellite peak is best performed at as small a field as possible - of course, $H$ should be large enough so that $\left|\mathbf{G}_{1}\right|$ is large enough to be outside the resolution window of the peak at $\mathbf{G}=0$. It is interesting to observe here that we can view the Bragg peak at $\mathbf{G}_{1}$ as arising from condensation at the nonzero $\mathbf{k}$ minimum in Fig. 9 of the dispersion of the exciton in the SC phase.

For completeness, we also show the real space form of the condensate $n_{H}(\mathbf{r})$ in Figs. 19 and 20 for two points in the

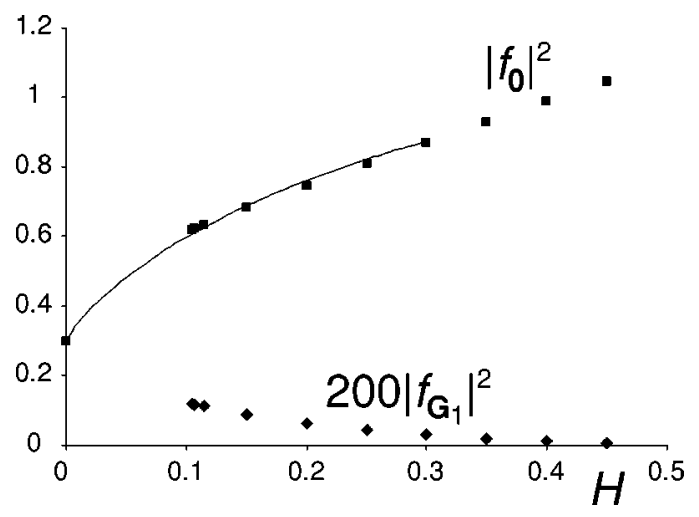

FIG. 18. As in Fig. 18 but for $s-s_{c}=-0.3$, showing $\left|f_{\mathbf{G}}\right|^{2}$ at $\mathbf{G}=0$ (squares) and at $\mathbf{G}=\mathbf{G}_{1}$ (diamonds). Unlike Fig. 18, the intensity $\left|f_{\mathbf{0}}\right|^{2}$ is nonzero even at zero field. The intensities at $\mathbf{G}$ $=\mathbf{G}_{1}$ have now been magnified by a factor of 200 . The line is 0.3 $+0.98 H \ln (2.12 / H)$, which is the best fit to the functional form in Eq. (5.11)

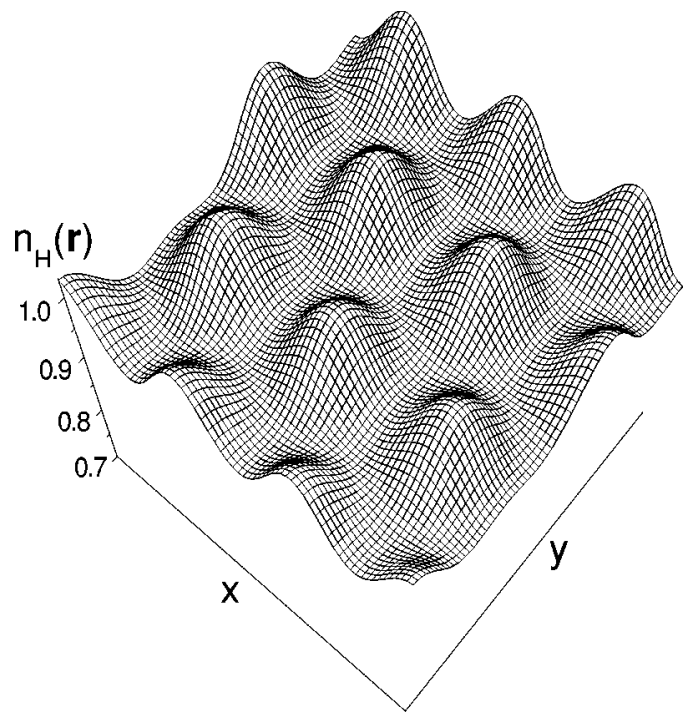

FIG. 19. Spatial form of the SDW order parameter $n_{H}(\mathbf{r})$ in the $\mathrm{SC}+\mathrm{SDW}$ phase at $s-s_{c}=-0.3, H=0.35$ (point $b$ in Fig. 6) over vortex lattice shown in Fig. 13. Notice that the vertical scale extends over a rather short range, and the modulation in $n_{H}(\mathbf{r})$ is quite small relative to the uniform component.

SC + SDW phase. The spatial form of the modulus of the superconducting order parameter for the first set of parameters is shown in Fig. 21. This last figure is the analog of Fig. 12 which was for the SC phase.

\section{E. Dynamic spin susceptibility}

Finally, we follow the presentation in Sec. IV D and discuss the dynamic spin spectrum in the SC+SDW phase. The nonzero $\omega$ spectral densities presented here appear along with the $\omega=0$ contributions in Eq. (5.9). We will restrict our attention to the susceptibility transverse to the ordering direction: this is given by the fluctuations of the last $N-1$ components in Eq. (5.1), which are in turn related to the Green's function $G_{H}$ in Eqs. (5.3) and (5.5). So the transverse dynamic spin susceptibility is given by a formula

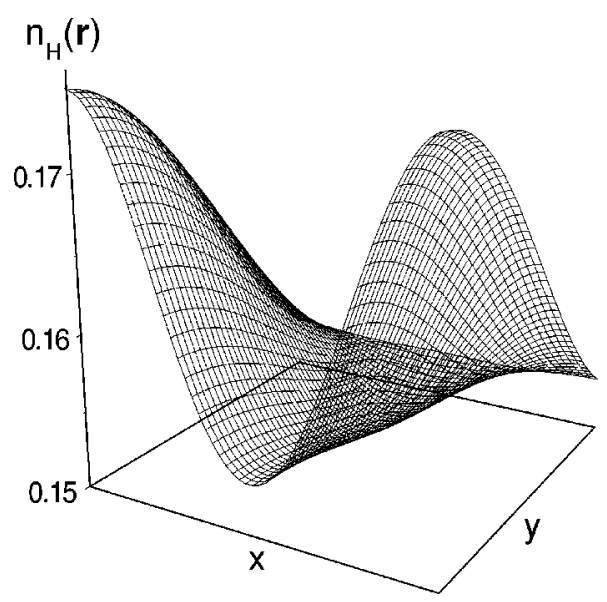

FIG. 20. As in Fig. 19; in the SC+SDW phase at $s-s_{c}=0.5$, $H=0.45$ (point $\mathrm{i}$ in Fig. 6) over a single vortex lattice unit cell shown in Fig. 13. 


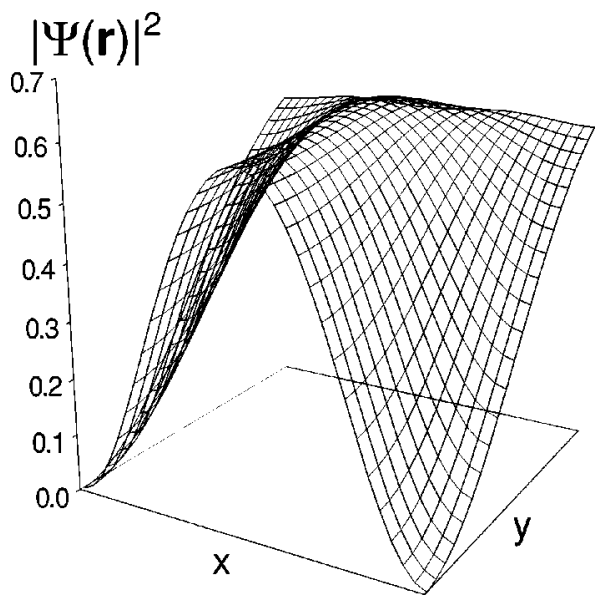

FIG. 21. Spatial dependence of the modulus of the superconducting order parameter $\left|\psi_{H}(\mathbf{r})\right|^{2}$ plotted on the rectangular half unit cell of the vortex lattice indicated by Fig. 13. As in Fig. 19, this result is for $s=s_{c}$ and $H=0.35$ (point b in Fig. 6).

analogous to Eq. (4.24). As before, we present the results by broadening the delta functions to sharp Lorentzians.

Our results for the spectral densities are shown in Figs. $22,23,24$, and 25 for a series of values of $s-s_{c}$ and $H$ in the $\mathrm{SC}+\mathrm{SDW}$ phase. Note first that there is always a gapless spin-wave mode. In addition there are features arising from scattering off the vortex lattice: these are strongest in the vicinity of the quantum critical point $A$ at zero field.

\section{OTHER PHASES IN ZERO MAGNETIC FIELD}

The next two sections involve a slight detour from the main flow of the paper. This section we will examine phases and phase transitions associated with composites or "fractions" of the primary order parameters $\Phi_{x, y \alpha}$ and $\phi_{x, y}$. This is done mainly for completeness. Readers not interested in this detour may skip ahead to next section without loss of continuity.

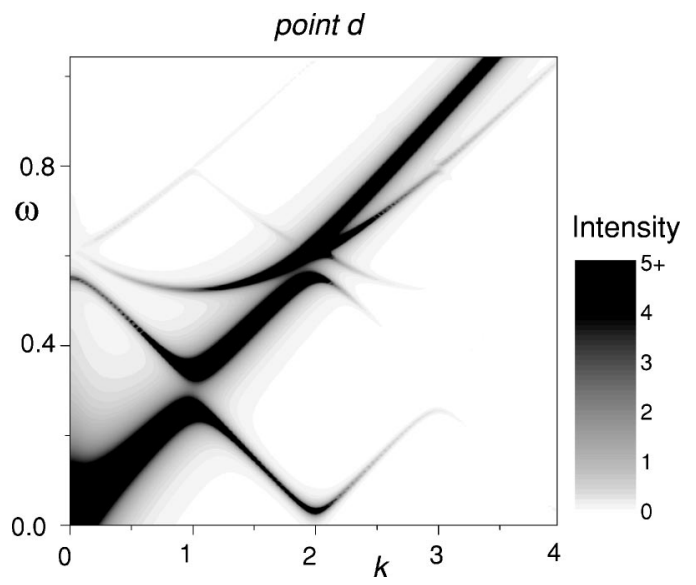

FIG. 22. As in Fig. 8, but for the transverse susceptibility in the $\mathrm{SC}+\mathrm{SDW}$ phase. The parameter values are $s=s_{c}$ and $H=0.05$ (point $d$ in Fig. 6).

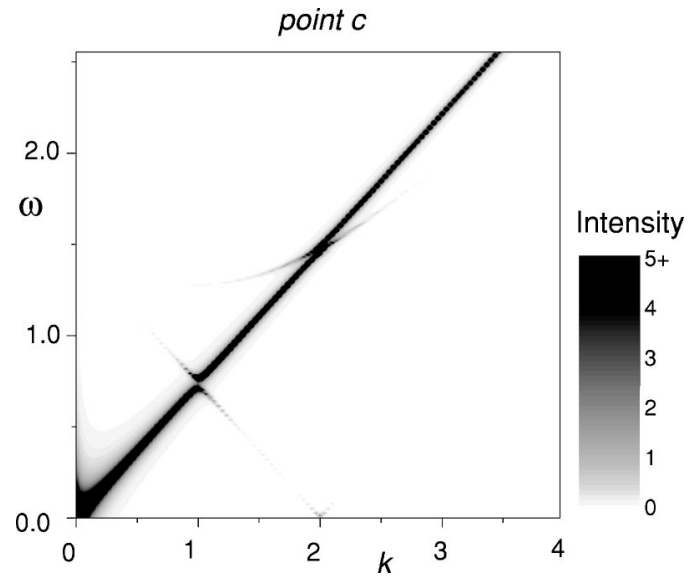

FIG. 23. As in Fig. 22 but for the values $s=s_{c}$ and $H=0.3$ (point $\mathrm{c}$ in Fig. 6).

\section{A. Phases with nematic order}

In Sec. I A we argued that a generalized non-twosublattice spin density wave order may be associated with a charge density wave. Another interesting possibility is that of spin nematic order, which has been previously discussed in Refs. 60-63. If the CDW order parameter may be understood as a spin zero combination of two $S_{\alpha}$ operators $\left[\delta \rho(\mathbf{r}, \tau) \sim S_{\alpha}^{2}(\mathbf{r}, \tau)\right]$, then the spin nematic order parameter $Q_{\alpha \beta}(\mathbf{r}, \tau)$ corresponds to their spin two combination

$$
Q_{\alpha \beta}(\mathbf{r}, \tau) \sim S_{\alpha}(\mathbf{r}, \tau) S_{\beta}(\mathbf{r}, \tau)-\frac{\delta_{\alpha \beta}}{3} S_{\alpha}^{2}(\mathbf{r}, \tau) .
$$

We pause briefly to also mention here an "Ising nematic" order which has also been considered recently. ${ }^{30}$ This order resides in real space associated with the lattice, and is distinct from the spin-space nematic order we are considering here. Order parameters with the Ising nematic order are $\left|\Phi_{x \alpha}\right|^{2}-\left|\Phi_{y \alpha}\right|^{2}$ and $\left|\phi_{x}\right|^{2}-\left|\phi_{y}\right|^{2}$, and these clearly measure a spontaneous choice between the $x$ and $y$ directions of the lattice. Our effective actions for $\Phi_{x, y \alpha}$ and $\phi_{x, y}$ are rich enough to also allow such orders.

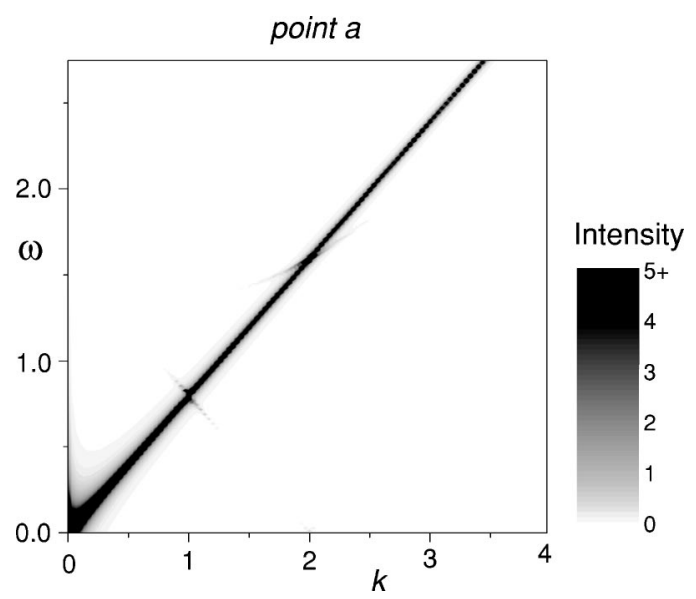

FIG. 24. As in Fig. 22 but for the values $s-s_{c}=-0.3$ and $H$ $=0.35$ (point a in Fig. 6). 


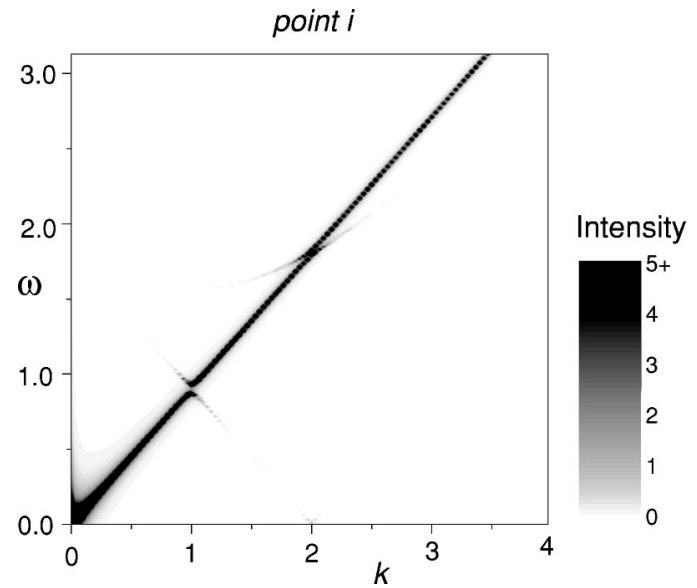

FIG. 25. As in Fig. 22 but for the values $s-s_{c}=0.5$ and $H$ $=0.45$ (point $\mathrm{i}$ in Fig. 6).

Returning to our discussion of spin nematic order in Eq. (6.1), we see that spin nematic order parameters that are consistent with the SDW order in Eq. (1.2) may be at wave vectors $(0,0)$ and $\mathbf{K}_{c x, y}$ :

$$
\begin{aligned}
Q_{\alpha \beta}(\mathbf{r}, \tau)= & Q_{0 \alpha \beta}(\mathbf{r}, \tau) \\
& +\operatorname{Re}\left[Q_{x \alpha \beta}(r, \tau) e^{i \mathbf{K}_{c x} \mathbf{r}}+Q_{y \alpha \beta}(r, \tau) e^{i \mathbf{K}_{c y} \mathbf{r}}\right] .
\end{aligned}
$$

It is natural to call $Q_{0 \alpha \beta}$ a uniform spin nematic order parameter, and $Q_{x, y \alpha \beta}$ a spin nematic density wave (SNDW). Both order parameters are symmetric $\left(Q_{i \alpha \beta}=Q_{i \beta \alpha}\right)$, but the uniform spin nematic $Q_{0 \alpha \beta}$ must be real, and the spin nematic density wave $Q_{x, y \alpha \beta}$ may be complex. The uniform spin nematic couples to the SDW order parameters $\Phi_{x, y \alpha}$ as

$$
\begin{aligned}
\mathcal{S}_{Q_{0}, \Phi}= & -\lambda_{1} \sum_{i=x, y} \int d^{2} r d \tau Q_{0 \alpha \beta} \\
& \times\left(\Phi_{i \alpha}^{\dagger} \Phi_{i \beta}+\Phi_{i \alpha} \Phi_{i \beta}^{\dagger}-\frac{2}{3} \delta_{\alpha \beta}\left|\Phi_{i \delta}\right|^{2}\right) .
\end{aligned}
$$

The spin nematic density wave $Q_{x \alpha \beta}(\mathbf{r}, \tau)$ couples to $\Phi_{x \alpha}$ via

$$
\mathcal{S}_{Q_{x}, \Phi_{x}}=-\lambda_{2} \int d^{2} r d \tau\left[Q_{x \alpha \beta}^{\dagger}\left(\Phi_{x \alpha} \Phi_{x \beta}-\frac{1}{3} \delta_{\alpha \beta} \Phi_{x \delta}^{2}\right)+\text { c.c. }\right]
$$

with a similar coupling between $Q_{y \alpha \beta}(\mathbf{r}, \tau)$ and $\Phi_{y \alpha}$.

The effective action for the spin nematic order parameters may be written from the analysis of the symmetries of Eq. (6.2). The interplay of the spin nematic and spin density wave orders may produce an extremely rich phase diagram. We will not attempt to explore its full richness, but restrict ourselves to the discussion of some simple illustrative examples. It is also worth pointing out that the appearance of the spin nematic order (either uniform or SNDW) does not give rise to the additional Bragg peak at zero energy, but produces a difference in the scattering cross sections for different neutron polarizations.

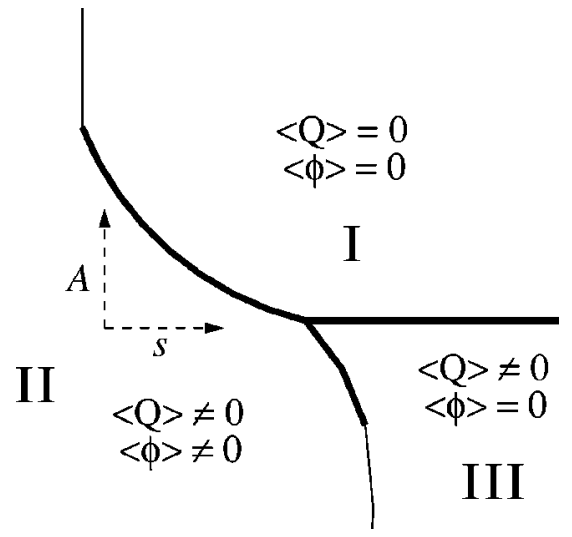

FIG. 26. Mean-field zero-temperature phase diagram of the model $\mathcal{S}_{\Phi}+\mathcal{S}_{Q_{0}}+\mathcal{S}_{Q_{0}, \Phi}$ in zero magnetic field for the case $u_{2}<0$.

\section{Uniform spin nematic}

To write the effective action for the uniform spin nematic $Q_{0 \alpha \beta}$ we can give essentially the same arguments as in deriving the Landau free energy for the classical nematics (see, e.g., Ref. 64)

$$
\begin{aligned}
\mathcal{S}_{Q_{0}}= & \int d^{2} r d \tau\left[\left(\partial_{\tau} Q_{0 \alpha \beta}\right)\left(\partial_{\tau} Q_{0 \beta \alpha}\right)+v_{Q}^{2}\left(\vec{\nabla} Q_{0 \alpha \beta}\right)\left(\vec{\nabla} Q_{0 \beta \alpha}\right)\right. \\
& +\frac{1}{2} A Q_{0 \alpha \beta} Q_{0 \beta \alpha}+\frac{1}{3} B Q_{0 \alpha \beta} Q_{0 \beta \gamma} Q_{0 \gamma \alpha} \\
& \left.+\frac{1}{4} C_{1}\left(Q_{0 \alpha \beta} Q_{0 \beta \alpha}\right)^{2}+\frac{1}{4} C_{2} Q_{0 \alpha \beta} Q_{0 \beta \gamma} Q_{0 \gamma \delta} Q_{0 \delta \alpha}\right] .
\end{aligned}
$$

By an appropriate spin rotation, the uniform spin nematic order parameter may always be brought into the diagonal form (this follows from the fact that it is a real and symmetric matrix)

$$
Q_{0 \alpha \beta}=\left(\begin{array}{ccc}
-\frac{1}{2}(q+\eta) & 0 & 0 \\
0 & -\frac{1}{2}(q-\eta) & 0 \\
0 & 0 & q
\end{array}\right) .
$$

When $\langle q\rangle \neq 0$ but $\langle\eta\rangle=0$ we have a uniaxial spin nematic, and when both expectation values are finite we have a biaxial spin nematic.

Let us start by considering the interplay of the uniform spin nematic with the collinear SDW (for simplicity we only consider one of the SDW orders, say $\Phi_{x \alpha}$ ). A schematic mean-field phase diagram at $T=0$ for $\mathcal{S}_{\Phi}+\mathcal{S}_{Q_{0}}+\mathcal{S}_{Q_{0}, \Phi}$ with $B \lambda_{1}<0$ and $u_{2}<0$ is shown in Fig. 26. Thick lines correspond to the first-order transitions, and thin lines correspond to the second order transitions. Phase I (SC) has no magnetic order of any kind; phase II ( $\mathrm{SC}+\mathrm{SDW}$ ) has commensurate SDW order, which is accompanied by a uniaxial spin nematic order; phase III $(\mathrm{SC}+\mathrm{UN})$ has a uniaxial spin nematic order. For $B \lambda_{1}>0$ and $u_{2}<0$ the phase diagram qualita- 


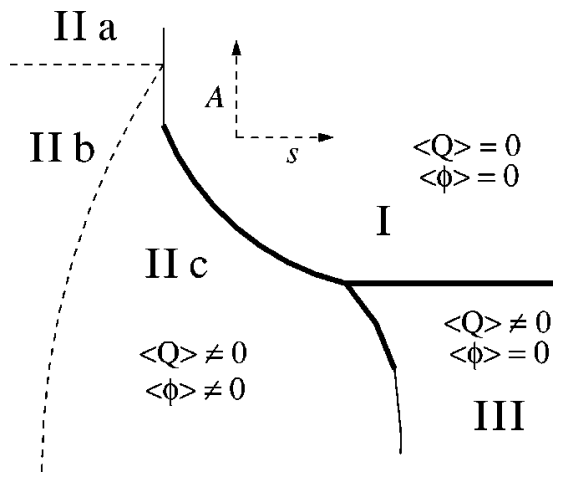

FIG. 27. Mean-field zero-temperature phase diagram of the model $\mathcal{S}_{\Phi}+\mathcal{S}_{Q_{0}}+\mathcal{S}_{Q_{0}, \Phi}$ in zero magnetic field for the case $u_{2}>0$.

tively remains the same, however, phase II has a finite expectation value of both $q$ and $\eta$ in Eq. (6.6), so it has an SDW order accompanied by the biaxial spin nematic order. A schematic phase diagram in the case $u_{2}>0$ is shown in Fig. 27. Phase II may now be a circular spiral SDW (IIa), an elliptic spiral SDW (IIb), and a collinear SDW (IIc).

\section{Spin nematic density wave}

For the spin nematic density wave the third order terms are prohibited by symmetry: they carry oscillating factors $e^{ \pm i \mathbf{K}_{c x, y} \mathbf{r}}$, and vanish after integrating over space in the long wavelength limit. Hence,

$$
\begin{aligned}
\mathcal{S}_{Q_{x}}= & \int d^{2} r d \tau\left[\left(\partial_{\tau} Q_{x \alpha \beta}^{\dagger}\right)\left(\partial_{\tau} Q_{x \beta \alpha}\right)+\widetilde{v}_{Q}^{2}\left(\vec{\nabla} Q_{x \alpha \beta}^{\dagger}\right)\left(\vec{\nabla} Q_{x \beta \alpha}\right)\right. \\
& +\frac{1}{2} \widetilde{A} Q_{x \alpha \beta}^{\dagger} Q_{x \beta \alpha}+\frac{1}{4} \widetilde{C}_{1}\left(Q_{x \alpha \beta}^{\dagger} Q_{x \beta \alpha}\right)^{2}+\frac{1}{4} \widetilde{C}_{2} Q_{x \alpha \beta}^{\dagger} \\
& \left.\times Q_{x \beta \gamma} Q_{x \gamma \delta}^{\dagger} Q_{x \delta \alpha}+\frac{1}{4} \widetilde{C}_{3} Q_{x \alpha \beta}^{\dagger} Q_{x \beta \gamma}^{\dagger} Q_{x \gamma \delta} Q_{x \delta \alpha}\right]
\end{aligned}
$$

and there is a similar action $\mathcal{S}_{Q_{y}}$.

The order parameter for the spin nematic density wave can be conveniently written using five complex numbers (see also Ref. 65)

$$
Q_{x \alpha \beta}=\left(\begin{array}{ccc}
-\frac{\psi_{x 1}}{\sqrt{3}}-\psi_{x 2} & \psi_{x 3} & \psi_{x 4} \\
\psi_{x 3} & -\frac{\psi_{x 1}}{\sqrt{3}}+\psi_{x 2} & \psi_{x 5} \\
\psi_{x 4} & \psi_{x 5} & \frac{2 \psi_{x 1}}{\sqrt{3}}
\end{array}\right)
$$

with normalization condition $\Sigma_{a=1, \ldots, 5}\left|\psi_{x a}\right|^{2}=1$. This representation makes obvious the connection between the order parameter for the spin nematic density wave and condensates of spin-2 particles, for which Ciobanu et al. ${ }^{66}$ argued that there exist three distinct phases (not related to each other by spin rotations), depending on the parameters $\widetilde{C}_{1}, \widetilde{C}_{2}$, and $\widetilde{C}_{3}$. The phase diagrams of the spin nematic density wave order vs the SDW order is similar to the case of uniform spin nematic (Figs. 26 and 27) with the main difference that the phase boundary between I and III is now second order.

\section{B. Exciton fractionalization}

Before concluding the section on the phases in zero field we would like to point out another interesting possibility for the system described by the generalizations of $\mathcal{S}_{\Phi}$. Consider this model in the regime where the spiral fluctuations are strongly suppressed, so we need to consider the collinear SDW order only; this happens in Eq. (1.8) for $u_{2}<0$ and with $\left|u_{2}\right|$ large. For simplicity we restrict our discussion to a SDW at wave vector $\mathbf{K}_{s y}, \Phi_{y \alpha}$. As discussed in Sec. I A, the collinear SDW can be written in the form (1.3), which we reproduce here for completeness:

$$
\Phi_{y \alpha}(\mathbf{r}, \tau)=e^{i \theta(\mathbf{r}, \tau)} n_{\alpha}(\mathbf{r}, \tau) .
$$

We also noted below Eq. (1.3) that such a separation of the physical order parameter $\Phi_{y \alpha}$ into the phase $\theta$ and the real vector $n_{\alpha}$ has an implicit ambiguity as we can simultaneously change the sign of both without altering $\Phi_{y \alpha}$. Formally this means that, for incommensurate $\mathbf{K}_{s y}$, the order parameter $\Phi_{y \alpha}$ belongs to the space $\left(S_{2} \times S_{1}\right) / Z_{2}$. For commensurate $\mathbf{K}_{s y}=2 \pi p^{\prime} /(p a)$, where $p^{\prime}, p$ are relatively prime integers, higher order terms not contained in Eq. (1.8) (but mentioned below it) imply $\theta$ prefers a discrete set of values $^{10,11}$ and the space is restricted to $\left(S_{2} \times Z_{p}\right) / Z_{2}$. Also, if full $\mathrm{SU}(2)$ spin rotation symmetry is absent, and the spins have an easy-plane restriction, then the first $S_{2}$ factor changes to $S_{1}$.

The $Z_{2}$ quotient in the order parameter space can be explicitly implemented as an Ising gauge symmetry, and it puts important constraints on the effective low energy theory. The lattice model consistent with such symmetry has the form

$$
\mathcal{S}_{I}=\sum_{\langle i j\rangle} J^{s} \sigma_{i j} n_{i \alpha} n_{j \alpha}+\sum_{\langle i j\rangle} J^{c} \sigma_{i j} \cos \left(\theta_{i}-\theta_{j}\right),
$$

where $i$ and $j$ are sites on the space-imaginary time lattice, the sum over $\langle i j\rangle$ extends over nearest neighbor links of this lattice, $J^{s}$ and $J^{c}$ are couplings imposing the propagation of SDW and CDW order, respectively, $n_{i \alpha}=n_{\alpha}\left(\mathbf{r}_{i}, \tau_{i}\right), \theta_{i}$ $=\theta\left(\mathbf{r}_{i}, \tau_{i}\right)$, and $\sigma_{i j}= \pm 1$ is an Ising gauge field that lives on the links of the lattice. One can easily see that the lattice action (6.10) is invariant under the $Z_{2}$ gauge transformation

$$
\begin{gathered}
n_{i \alpha} \longrightarrow \sigma_{i} n_{i \alpha}, \\
\theta_{i} \rightarrow \theta_{i}+\frac{\pi}{2}\left(1-\sigma_{i}\right), \\
\sigma_{i j} \rightarrow \sigma_{i} \sigma_{i j} \sigma_{j}
\end{gathered}
$$

for $\sigma_{i}= \pm 1$.

Models of the kind (6.10) have been discussed earlier in various contexts. ${ }^{63,67-71}$ It was pointed out, for example, that another term allowed by symmetry is a Maxwell term for the lattice gauge field 


$$
\mathcal{S}_{\sigma}=-K \sum_{\square}\left[\prod_{\square} \sigma_{i j}\right],
$$

where the sum on $\square$ extends over the plaquettes of a (2 $+1)$-dimensional lattice. Such a term may be generated by integrating out the high energy degrees of freedom or may be present due to certain frustrating terms in the original microscopic Hamiltonian. ${ }^{69-71}$ This term has a striking effect on the properties of the model (6.10): it gives rise a phase in which the exciton $\Phi_{y \alpha}$ fractionalizes, and fluctuations of $n_{\alpha}$ are separated from the fluctuations of $\theta$. Loosely speaking, the SDW and the CDW fluctuations get decoupled.

It is useful to discuss the consequence of the confinementdeconfinement in the symmetric phase in which global symmetries are preserved: the models of this paper are invariant under $\mathrm{SU}(2)$ spin rotations, and the sliding $\mathrm{U}(1)$ symmetry [for commensurate values of $\mathbf{K}_{s y}$, the $\mathrm{U}(1)$ symmetry is reduced to a discrete $Z_{p}$ "clock" symmetry, but essentially unchanged considerations apply nevertheless $\left.{ }^{10,11}\right]$. The immediate manifestation of the confinement-deconfinement transition in such a symmetric phase is the change in the degeneracy of the lowest-energy excitations. In the confining phase their degeneracy is 6: this six-fold degenerate excitation corresponds to the quanta of the exciton field $\Phi_{y \alpha}$, which have six real components. In contrast, in the deconfining phase we have separate excitations with degeneracies of 3 and 2, corresponding to quanta of $n_{i \alpha}$ and $\theta_{i}$, respectively. This may be understood by noting that the unbroken symmetry ground state of the model $\mathcal{S}_{I}+\mathcal{S}_{\sigma}$ is a singlet ground state of the $\mathrm{SO}(3) \times \mathrm{SO}(2)$ rotors, where in the confining phase the angular momenta of the two rotors $\left(l_{1}, l_{2}\right)$ $=\left(L_{\mathrm{SO}(3)}, L_{\mathrm{SO}(2)}\right)$ are bound by the constraint $l_{1}+l_{2}$ $=$ even, but this constraint is not present in the deconfining phase. Hence, in the confining phase the lowest excitation has $\left(l_{1}= \pm 1, l_{2}=1\right)$, which gives the degeneracy of 6 . In the deconfining phase we can have excitations $\left(l_{1}= \pm 1, l_{2}=0\right)$ and $\left(l_{1}=0, l_{2}=1\right)$, and these have degeneracies 3 and 2 , respectively. We point out that the exact degeneracy of $l_{1}=$ \pm 1 states requires the absence of the Berry's phase for the the $\mathrm{SO}(2)$ rotor, and comes from the inversion symmetry of the system, as was noted below Eq. (1.8). It is not related to the possible particle-hole symmetry of the underlying microscopic model.

It is worth emphasizing that the exciton fractionalization discussed above has a very different physical interpretation from that of electron fractionalization discussed in "RVB" theories of doped Mott insulators: ${ }^{69}$ in the latter there are elementary $S=1 / 2$ spinons which do not appear in our fractionalized states above. Instead our exciton fractionalization is within the sector of spin and charge density waves, and the collective spin excitations only have integer spin.

Zaanen et al..$^{72}$ have recently discussed fractionalization in a microscopic picture of spin and charge order in "fluctuating stripe" states: the physical content of their analysis is quite similar to that of our discussion above. However their proposed effect action does not include the CDW phase field $\theta_{i}$, and we believe this is essential for a complete description of stripe physics.

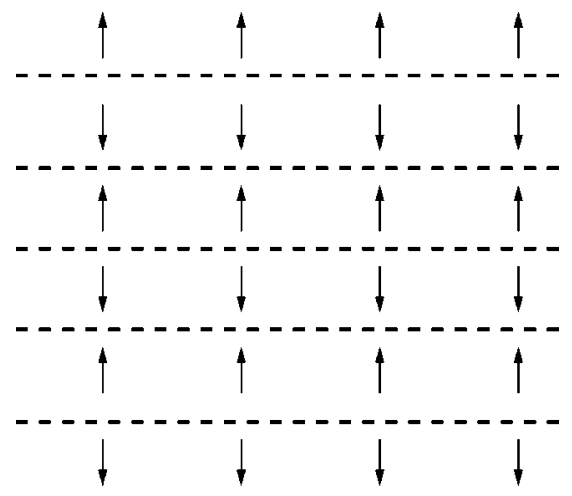

FIG. 28. A schematic picture of the non-two-sublattice collinear SDW order and associated CDW as a periodic array of antiphase domain walls in Néel order at $(\pi / a, \pi / a)$. Arrows show the change of sign of the Néel order across a hole rich domain wall. The fields $n_{i \alpha}$ and $\theta_{i}$ are space independent in the above configuration.

We have implicitly assumed above that the exciton fractionalization transition occurs in a background of SC order. However, a similar transition is also possible within a Fermi liquid. We believe that such a quantum critical point is a promising candidate for describing the finite temperature crossovers in the normal state of the cuprates. Ordinary SDW/CDW transitions in a Fermi liquid ${ }^{28}$ have the unsatisfactory (in our view) feature of flowing to a free field fixed point because they are in their upper-critical dimensions. In contrast, the exciton fractionalization transition may well remain strongly coupled even in the presence of Fermi surface. Corresponding speculations of fractionalization influencing finite temperature quantum criticality were also made by Zaanen et al. ${ }^{72}$ Again, their and our proposals should be distinguished from those associated with electron fractionalization made in, e.g., Ref. 73.

\section{Topological defects}

An alternative picture of fractionalization, and of the various order parameters above, may be given in the language of the topological defects of the SDW phase; the condensation of distinct defects in the SDW state distinguishes the new phases that appear. To simplify the presentation of this subsection we will describe the case of an easy plane antiferromagnet, in which the vectors $\Phi_{i \alpha}=e^{i \theta_{i}} n_{i \alpha}$ may only be in the $x-y$ plane, but will also state the results for systems with full $\mathrm{SU}(2)$ spin rotation symmetry. A related discussion of defects in SDW states also appears in Ref. 74.

We start by giving a simple cartoon ${ }^{22,72,75}$ of the non-twosublattice SDW order $\Phi_{y \alpha}=$ const and the associated CDW in Fig. 28. Hole rich stripes (indicated by the dashed lines) act as antiphase domain walls for the hole poor antiferromagnetic domains. The Néel order shown by arrows changes sign when crossing such domain walls (the Néel order should not be confused with the vector $n_{i \alpha}$ which appears in the definition $\Phi_{i \alpha}=e^{i \theta_{i \alpha}} n_{i \alpha}$; the former oscillates as shown in Fig. 28, while $n_{i \alpha}$ is constant in this configuration.).

Schematic pictures of the topological defects of the collinear SDW state are shown on Figs. 29-31 with crosses indicating the locations of the centers of defects (see also 


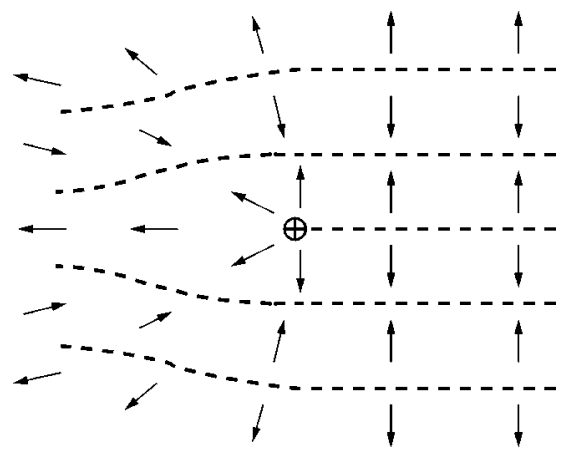

FIG. 29. Elementary topological excitation of the collinear SDW phase: a composite of $1 / 2$ vortex in $\theta_{i}$ and $\pi$ disclination in $n_{i \alpha}$. Both $e^{i \theta_{i}}$ and $n_{\alpha}$ change sign when going around this topological defect, but the physical order parameter $\Phi_{y \alpha i}=e^{i \theta_{i}} n_{i \alpha}$ is single valued.

Ref. 72). These defects can also be formally classified by computing the homotopy groups; for systems with an easyplane spin symmetry the relevant homotopy group ${ }^{76}$ is $\pi_{1}\left[\left(S_{1} \times S_{1}\right) / Z_{2}\right]=Z \times Z$, while for full $\mathrm{SU}(2)$ spin symmetry it is $\pi_{1}\left[\left(S_{2} \times S_{1}\right) / Z_{2}\right]=Z$. These mathematical statements actually obscure some of the physical content, as will become clear from our discussion below.

We first discuss the physical content of the defect classification for the easy-plane case. Consider the most elementary topological defect: this is a composite of a $1 / 2$ vortex for the phase $\theta_{i}$ and a $\pi$ disclination (i.e., $1 / 2$ a meron) for the vector $n_{i \alpha}$ (see Fig. 29); this defect is also a central actor in the discussion of Zaanen et al. ${ }^{72}$ When circling around such a defect both $e^{i \theta}$ and $n_{i \alpha}$ change sign, however, the physical order parameter $\Phi_{i \alpha}=e^{i \theta_{i}} n_{i \alpha}$ is uniquely defined. Given the circulations in $\theta_{i}$ and $n_{i, \alpha}$, we label this defect $(1 / 2,1 / 2)$. Actually, we can make four such elementary defects by changing the signs of the circulation of $\theta_{i}$ and $\pi$ disclination and taking all of such combinations: we label these as $( \pm 1 / 2, \pm 1 / 2)$ in an obvious manner. Pairs of such elementary defects may be combined to give a full vortex for $\theta$, which is trivial in the $n_{i \alpha}$ sector [see Fig. 30; this is the defect $(1,0)]$ and a meron for the $n_{i \alpha}$, that it is trivial in the

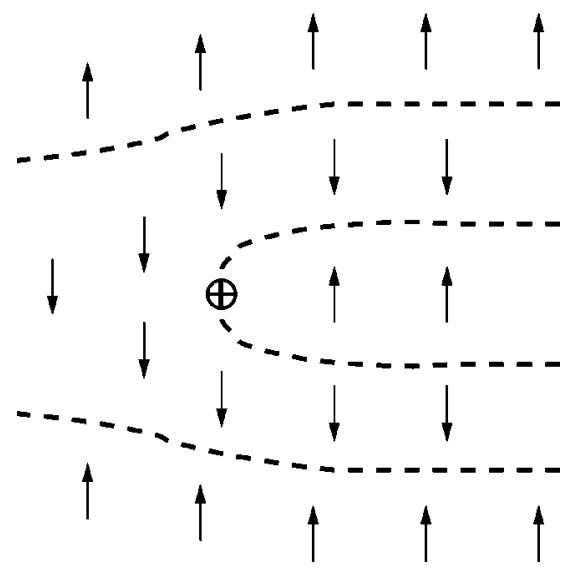

FIG. 30. Elementary topological excitation of the collinear SDW phase: a vortex in $\theta_{i}$. The circulation of $\theta$ is equal to $2 \pi$.

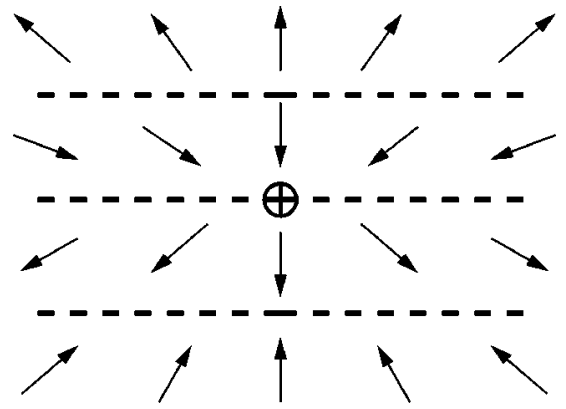

FIG. 31. Elementary topological excitation of the collinear SDW phase: a meron of $n_{i \alpha}$. Such an object is stable only in systems with an easy-plane symmetry. Far away from the vortex core $n_{i \alpha}$ winds in the plane similar to a usual vortex. Closer to the vortex center it may acquire an out of plane component. Systems with full $\mathrm{SU}(2)$ spin rotation symmetry only have pointlike, instanton defects in spacetime: hedgehogs.

$\theta_{i}$ sector [see Fig. 31; this is the defect $\left.(0,1)\right]$. Continuing in this manner, we see that all defects are labeled $\left(m_{1} / 2, m_{2} / 2\right)$ with $m_{1}, m_{2}$ integers such that $m_{1}+m_{2}$ is even. These labels lie on the analog of a FCC lattice in two dimensions; this is equivalent to a square lattice after a rotation by $45^{\circ}$, and hence the homotopy group is $Z \times Z$. This mathematical statement hides the fact that there is a fundamental physical difference between the $( \pm 1 / 2, \pm 1 / 2)$ and the $(1,0),(0,1)$ defects, which we have discussed above.

Next we turn to the case with full SU(2) symmetry. Now the $1 / 2$ meron in $n_{i \alpha}$ is actually equivalent to the $-1 / 2$ meron (they are both better called $\pi$ disclinations), and so there is no distinction between $(1 / 2,1 / 2)$ and $(1 / 2,-1 / 2)$; moreover, the $(0,1)$ defect is topologically trivial. Consequently the spacetime line defects can simply be labeled $m_{1} / 2$, where $m_{1}$ is an integer representing the phase winding of $\theta_{i}$, and hence the homotopy group is $Z$. However, there continues to be a fundamental physical distinction between the cases with $m_{1}$ odd and even. For $m_{1}$ odd, there must be a corresponding $\pi$ disclination in $n_{i \alpha}$, while for $m_{1}$ even the $n_{i \alpha}$ configuration can be constant. The $\mathrm{SU}(2)$ case also has point defects in spacetime, the "hedgehogs," which proliferate at spin disordering transitions.

The various phases discussed above can be easily understood using the picture of topological defect condensation in a phase with conventional SDW order (the SC+SDW phase).

When the elementary $1 / 2$ vortex- $\pi$ disclination composites condense we have a conventional (unfractionalized) disordered phase (the SC phase).

When vortices and merons (or hedgehogs) condense, but $1 / 2$ vortex- $\pi$ disclination composites remain gapful excitations, we find exciton fractionalization as discussed above. The uncondensed $1 / 2$ vortex- $\pi$ disclination composites correspond to the finite energy "visons" 69 of the fractionalized phase of the $Z_{2}$ gauge theory.

When only the merons (or hedgehogs) condense we find the CDW phase with no spin order.

When only the $\theta$ vortices condense we get the spin nematic phase with no CDW order. 


\section{EARLIER WORK ON SC AND SDW ORDERS}

As we noted earlier, this section is a detour from the main flow of arguments in this paper. For completeness, we review earlier theoretical and experimental work on the interplay of magnetism and superconductivity, and discuss connections to our treatment here. Less specialized readers may skip ahead to the conclusions if they wish.

Early neutron-scattering measurements ${ }^{77,78}$ of the evolution of the magnetic order in $\mathrm{La}_{2}-\delta(\mathrm{Sr}, \mathrm{Ba}){ }_{\delta} \mathrm{CuO}_{4}$ with $\delta$ observed spectra which were interpreted ${ }^{79}$ as evidence for the proximity of a quantum critical point at which the SDW order vanished, and which obeyed strong hyperscaling properties. It was proposed ${ }^{79,80,35}$ that such a quantum critical point (with dynamic exponent $z=1$ ) controlled physical properties over a range of doping concentrations. Further support for such a proposal appeared in the NMR experiments of Imai and collaborators ${ }^{81}$ which displayed crossovers characteristic of the vicinity of a magnetic quantum critical point, with the critical point at a doping concentration $\delta=\delta_{c} \approx 0.12$; similar evidence was presented recently by Fujiyama et $\mathrm{ll}^{82}$ (for a review of the NMR data in this context, see Ref. 4). The concentration $\delta_{c}=0.12$ is well within the superconducting phase, and so the magnetic transition takes place within a background of superconducting order, i.e., there is a second order transition between a phase with coexisting SC and SDW order (the SC + SDW phase) and an ordinary superconductor (the SC phase). As we noted in Sec. $\mathrm{I}$, the neutron-scattering measurements of Aeppli et al. ${ }^{3}$ at $\delta=0.14$ provided rather direct evidence for such a magnetic quantum critical point. Additional evidence for microscopic coexistence of SC and SDW orders has appeared in a number of recent experiments. ${ }^{12,13,20,83-87}$

[For completeness, we also note here the additional phases present at very low $\delta$ which were not the subject of study in this paper: in $\mathrm{La}_{2}-{ }_{\delta} \mathrm{Sr}_{\delta} \mathrm{CuO}_{4}$, the three-dimensional, two-sublattice, insulating Néel state is present for $\delta<0.02$, and is followed by an insulating SDW state with its wave vector polarized along the diagonal $(1, \pm 1)$ directions. ${ }^{1,2}$ As noted in Sec. I, at $\delta=0.055$ there is a first-order insulator-tosuperconductor transition to the SC+SDW phase, ${ }^{1,2}$ which has the SDW oriented along the $(1,0),(0,1)$ directions; we discussed the properties of this $\mathrm{SC}+\mathrm{SDW}$ phase in this paper.]

A significant implication of the existence of a magnetic critical point at $\delta=\delta_{c}$ is that remnants of the magnetic excitations should be visible in the SC phase at $\delta>\delta_{c}$. As originally discussed in Ref. 35, for such critical points there is a sharp, gapped $S=1$ collective mode (a spin exciton) which would appear as a "resonance" in the neutron-scattering cross section. This resonance should appear at the SDW ordering wave vector in (1.1), and recent evidence for gapped, low energy spin excitations at such a wave vector is in Refs. 88,89 . Strong resonant scattering is also seen at the Néel order wave vector $(\pi, \pi)$ in the SC phase: ${ }^{88,91-93}$ this remains at relatively high energies and may be viewed as a remnant of commensurate correlations at short length scales. ${ }^{10}$ Batista et al.$^{90}$ have argued that the strong gapped response at $(\pi, \pi)$ is due to the superposition of the response at the two neighboring SDW ordering wave vectors at $\pm \vartheta$ in Eq. (1.1).

Another perspective on this quantum critical point, which was useful in our analysis, was provided by Zhang's $\mathrm{SO}(5)$ theory. ${ }^{32}$ This theory goes beyond the picture of competing SC and SDW orders in the ground state and adopts a stronger assumption of a microscopic dynamic symmetry between them; this has been supported by analytic ${ }^{94-97}$ and numeric ${ }^{98,99}$ studies of a number of models. The generator of the enlarged $\mathrm{SO}(5)$ symmetry is the $\pi$ excitation, a $S=1$ collective mode with charge 2 and momentum $(\pi, \pi) \cdot{ }^{94,95} \mathrm{~A}$ sharp distinction between the models with and without the $\pi$ excitation is possible in the weak interaction limit of a generalized BCS-RPA theory, where by going to the normal state one can check for the existence of a sharp collective mode with the quantum numbers of the $\pi$ particle. ${ }^{100}$ However, a clear distinction is absent in the physically relevant strong coupling regime. For example, in the SC phase charge is only conserved modulo 2 , and this charge 2 particle is in fact indistinguishable from the neutral $S=1$ exciton in earlier theories ${ }^{35}$ of the SDW ordering transition (see also Ref. 101). This exciton is smoothly connected to the $S=1$ excitation in a paramagnetic Mott insulator, ${ }^{102}$ and an interpretation of its "resonance peak" as a generator of $\mathrm{SO}(5)$ rotations does not hold. In zero applied magnetic field, it is possible to formulate a theory of the exciton, ${ }^{8,35}$ and the associated SDW fluctuations, without any explicit reference to the SC order; the SC correlations only serve to modify various couplings in the effective action for the SDW order. What we abstract from the analysis of Zhang ${ }^{32}$ is the idea that the strength of the SC order itself should be viewed as a parameter which tunes the system across the magnetic quantum critical point: this emphasizes a local competition between the SC and SDW orders.

We also mention that these $\mathrm{SO}(5)$ models naturally describe a competition between the SC and the two sublattice SDW (Néel) phases. Non-two-sublattice SDW can then appear as a result of the competition between phase separation and long range Coulomb interaction, ${ }^{103,104}$ across a firstorder transition from the SC to the SDW phase. In this paper we will describe effective models for the non two-sublattice SDW directly, across a second-order transition from the SC to $\mathrm{SC}+\mathrm{SDW}$ state.

The precise nature of the interplay of SC and SDW orders in the cuprates at nonzero temperatures in three-dimensional models been a controversial subject (this paper dealt with two-dimensional quantum models at $T=0$, and so the issues in this paragraph are only peripherally related to our main discussion). Following earlier general analyses, ${ }^{105}$ Zhang $^{32}$ pointed out four generic possibilities for the phase diagram, proposed the appearance of exact $\mathrm{SO}(5)$ symmetry in the classical theory of a finite temperature bicritical point (this symmetry is actually only present in the equal-time correlators ${ }^{106}$ ), and suggested that this is the situation most likely realized for the cuprates. In the presence of such a bicritical point, there is a first-order transition between the $\mathrm{SC}$ and SC+SDW phases at low temperatures, and the energy of the exciton (or $\pi$ particle) remains relatively large in the SC phase. The possibility of a critical point that is best 
described as corresponding to the regime exactly on the border between the bicritical and tetracritical behavior was suggested in Ref. 107 [the projected SO(5) models discussed in that paper lead to such fine tuning for the effective theories]. Other critical points, including a tricritical one, have been suggested recently by Kivelson et al. ${ }^{108}$ We have argued here, instead, that many features of the experiments require the energy of the exciton to vanish at a quantum critical point describing a second order transition between the SC and $\mathrm{SC}+\mathrm{SDW}$ phases; this appears when the finite temperature multi critical point is tetra critical (i.e., the four phases SC, $\mathrm{SDW}, \mathrm{SC}+\mathrm{SDW}$, and "normal" all meet at one finite temperature point) and has strongly broken equal-time $\mathrm{SO}(5)$ symmetry. We also note that Aharony ${ }^{109}$ has recently shown, by an exact renormalization group analysis of fluctuations, that the finite temperature multicritical point has a "decoupled" structure, which does indeed exhibit tetracritical behavior. A finite coexistence region between the superconducting and antiferromagnetic phases in the cuprates has been also recently discussed by Martin et al. ${ }^{110}$

We have also mentioned the recent study of Kivelson et al. ${ }^{108}$ of a variety of finite temperature multicritical phase diagrams in three dimensions involving the SC and SDW order parameters. They pay particular attention to the possibility of a two-phase coexistence of SC and SDW order parameters, which should be distinguished from the homogenous $\mathrm{SC}+\mathrm{SDW}$ phase we have discussed in this paper. In the presence of a finite field in the two-phase coexistence case, we would expect that the SC component has a $H \ln (1 / H)$ term in its free energy, while the SDW component only has an analytic $H^{2}$ correction. Consequently, with increasing $H$, the fraction of the SDW component will grow at the expense of the SC component with an $H \ln (1 / H)$ dependence.

We mention that several other proposals for the experimental consequences of the competition between the SC and SDW orders in the cuprates may be found in Refs. 111-115, and 116.

\section{CONCLUSIONS}

The primary purpose of this paper has been a description of the phase diagram in Fig. 3 and of the static and dynamic properties of its low field phases. The point of departure of our work was the existence of a second-order quantum transition between the SC and SC+SDW phases in zero applied magnetic field (our methods can also be extended to weakly first-order transitions, but we did not discuss this here): we reviewed in Sec. I the early theoretical proposals and the experimental evidence in support of such a transition. In a nonzero field we found that this transition extended into a line of second-order transitions indicated by AM in Fig. 3. This transition line approaches the $H=0$ axis with a vanishing derivative, which implied that relatively small fields could have a significant effect on the low energy spin fluctuation spectrum: this is our qualitative explanation for the field-induced enhancement of low-energy SDW correlations observed by Lake et al. ${ }^{12}$ Our analysis also showed that the critical properties of the transition in finite field were in all cases described by the familiar $\mathrm{O}(3)$ symmetric $\varphi^{4}$ field theory: these have already been described in some detail in earlier work. ${ }^{7,35}$ This mapping to the simple $\mathrm{O}(3)$ continuum field theory occurs when the spin correlation length becomes larger than the vortex lattice spacing (as is always the case close enough to $\mathrm{AM}$ ), and accounts for the fact that $\mathcal{S}_{\text {lat }}$ pins the charge order fluctuations and so reduces the order parameter to a real, three-component vector. In principle, the Zeeman coupling to the $\mathrm{O}(3)$ field theory modes should also be included in the asymptotic critical region, but existing work $^{7,117}$ has shown how to do this. We believe that experimental discovery of the critical field along the phase boundary $\mathrm{AM}$ is an exciting possibility for future investigations. Such a study should begin with a sample with its $s$ value slightly larger than $s_{c}$; application of a field should then allow tuning of the system across the quantum critical behavior associated with the AM phase boundary. The precise experimental control available over the value of $H$ should allow unprecedented access to an interesting, interacting quantum critical point in two dimensions. In the following subsection we discuss a number of very recent experimental studies, and compare them to our results to the extent possible: we also mention proposals for future experiments.

\section{Implications for experiments}

So far, the most direct connection of our results with experiments is provided by neutron-scattering measurements of the field dependence of the ordered moment in the SC + SDW phase. Two such experiments have been performed $^{13,14}$ in different but related compounds, and both show a reasonable fit to the predicted ${ }^{9} H \ln (1 / H)$ dependence. The experiment of Khaykovich et al..$^{13}$ appears to be in a parameter regime similar to that of Fig. 18: there is an appreciable ordered moment at zero field, and the elastic scattering intensity roughly doubles in a field about a quarter of $H_{c 2}$. This is an important consistency check on our entire approach, as all numerical parameters in our computation had physically reasonable values. As is clear from Fig. 18, the intensity of the satellite peaks associated with the reciprocal lattice vectors of the vortex lattice is quite small for these parameters: this explains why such a satellite peak was not seen in the experiments even though they had the requisite wave vector resolution. The experiments of Lake et al. ${ }^{14}$ are in a regime similar to that of Fig. 17: they had quite a small moment at zero field, but this grew rapidly with field with a clear $H \ln (1 / H)$ dependence. Again, as Fig. 17 shows, the satellite vortex lattice peaks have a very small intensity, and this is presumably why they were not observed. This experimental sample appears to be rather close to $s=s_{c}$, and we hope that a future experiment will move just past $s_{c}$ and study the transition across the AM phase boundary in Fig. 3 .

Our theoretical computations also suggest an approach by which the vortex (reciprocal) lattice may be detected in the spin fluctuation spectrum. While its influence on the elastic Bragg peaks ${ }^{32,33,9,37}$ was found to be very small in Figs. 17 and 18, the spectra in Figs. 8-10 and 22 show a more significant influence in the inelastic neutron-scattering cross section. These plots may be viewed as the "band structure" of the exciton moving in the vortex lattice, and the exciton dispersion shows clear features at the Bragg reflection planes 
in the reciprocal lattice of the vortex lattice. So we predict that a careful study of the inelastic-neutron-scattering spectrum may more easily yield evidence for the presence of the vortex lattice.

Next, we turn to the recent STM measurements of Hoffman et al. ${ }^{18}$ These authors have observed signals of charge order in the vortex lattice of BSCCO in the electron density of states at subgap energies. The charge order is at wave vectors $\mathbf{K}_{c x}=[\pi /(2 a), 0]$ and $\mathbf{K}_{c y}=[0, \pi /(2 a)]$ (period of four lattice spacings), is peaked at the vortex cores, and extends about to a distance which is about a quarter of the inter-vortex spacing. These measurements are most likely in the SC phase, where the SDW order is dynamically fluctuating. The nucleation of charge order by vortices in such a phase (but with the spins remaining dynamic) was predicted in Refs. 10, 51. Lattice scale theories ${ }^{26}$ of charge order in superconductors with preserved spin rotation invariance also found a substantial doping range of bond-centered charge order with a period of four lattice spacings, as did density matrix renormalization group studies. ${ }^{52}$ The spatial extent of the envelope of this charge order in the SC phase has been computed in the present paper: the length scale in the observations is quite similar to that in our computations in Figs. 15 and 16. These computations were carried out for the same set of parameters (only the value of $s-s_{c}$ was changed to tune the doping level) used to obtain general quantitative consistency with the neutron-scattering experiments above. The data of Hoffman et al. seems rather similar to the result for $\Omega(\mathbf{r})$ at point $k$ in Fig. 16, and the location of this point in the phase diagram of Fig. 6 is very reasonable, given the optimal doping of their sample and of their $H$ value. This agreement suggests to us that the system studied by Hoffman et al. has dynamic spin excitons, above a spin gap, which extend throughout the vortex lattice, as in Figs. 15 and 16; the charge order is then a signal of the pinning of these excitons by terms like those in $\mathcal{S}_{\text {lat }}$. An alternative model, in which the spin order was confined only to the region where charge order has been observed in STM, would have difficulty explaining the neutron-scattering experiments: spin order so confined should yield easily observable satellite elastic Bragg peaks at the wave vectors of the reciprocal of the vortex lattice.

Our computations also offer explanations for other features of the STM data which would be difficult to understand in terms of charge order nucleated independently in each vortex core: there is a noticeable correlation between the phase and orientation of the charge order between different vortices, which extends across the entire experimental sample. We believe this correlation is induced by the extended spin exciton states above the spin gap. Our model for the STM experiments can therefore be summarized as follows: the superflow in the vortex lattice reduces the energy of extended spin exciton states, and the sliding degree of freedom associated with spin density is then pinned by the vortex cores; this results in static CDW around each vortex, but the SDW order remains dynamic and gapped. A particular strength of our model is that it consistently explains the STM and neutron-scattering experiments using the same set of parameters.
For the future, our theory suggests that neutron-scattering and STM studies of SDW/CDW order should be carried out in systems where a uniform superflow has been induced directly by a current source, with no magnetic field penetrating the sample. This will eliminate the vortex cores, but the superflow should still enhance the tendency for SDW/CDW order. Charge order can be pinned near impurities/defects of various kinds (e.g., dislocations, grain boundaries, surfaces), and so become visible to STM.

We briefly comment on the high field phases (SDW and "normal") in Fig. 3, in which superconductivity is destroyed by the magnetic field. This regime may be of relevance to the experiments of Boebinger et al. ${ }^{118}$ Dynamic fluctuations of the superconducting order surely become important as we approach these phases, and so the theory of the present paper is not complete. Nevertheless, given the nucleation of charge order near the vortex cores in the SC phase (and its observation in the STM experiments ${ }^{18}$, it is natural to presume that this charge order survives into the "normal" phase. The transport properties of the nonsuperconducting phases remain a very interesting topic for future research, but our naive expectation is that they are insulators.

Another interesting type of experiments on superconductors in the vortex state has been performed recently by Curro et al. ${ }^{15}$ and Mitrović et al. ${ }^{16,17}$ They measured the local field dependence of the ${ }^{17} \mathrm{O}$ spin-lattice relaxation rate $\left(1 / T_{1}\right)$ and spin-echo decay rate $\left(1 / T_{2}\right)$, this allowed them to deduce the rates as a function of position in the vortex lattice. Below we suggest how these experiments can be interpreted in our picture of the mixed state of the cuprates. The spin-lattice relaxation rate $1 / T_{1}$ measures the rate at which nuclear spins are overturned as a result of interaction with electron spins. In the BCS picture of vortices in a $d$-wave superconductor, ${ }^{15,16,119,120}$ this quantity is proportional to $N(0)^{2}$ and therefore increases dramatically close to the vortex cores due to suppression in the superconducting gap. On the other hand, as discussed in detail earlier in this paper, for the not too overdoped cuprates, charge density waves are nucleated around the vortex cores, which should lead to a suppression in the local quasiparticle density of states, and hence $1 / T_{1}$. This effect appears to have been observed in the experiments of Ref. 16. Another mechanism for the nuclear spin relaxation is via the collective excitations of the electron system. In particular, the excitonic SDW excitations provide a large number of low energy $S=1$ excitations for flipping the nuclear spins. We suggest that a strong increase in the the high field part of $1 / T_{1}$ (corresponding to the vortex cores) with increasing magnetic field in the experiments of Mitrovic et al. ${ }^{16}$ reflects the growth of the SDW correlations and the corresponding increase in the excitonic susceptibility. It would be interesting to study this enhancement quantitatively and compare it with the $H \ln (1 / H)$ behavior observed in neutron-scattering experiments and derived theoretically in this work. We mention that the non-two-sublattice SDW makes this mechanism more effective for relaxing the ${ }^{17} \mathrm{O}$ nuclear spins, in contrast to the $(\pi, \pi)$ electron magnetism which leads to a magnetic field on the oxygen sites only through the Dzyaloshinskii-Moriya interaction and weak ferromagnetism. The echo decay rate $1 / T_{2}$ is related to the in- 
homogeneity of the local magnetic fields. The appearance of the local SDW order (or sufficiently slow fluctuations) should therefore contribute to the increase in $1 / T_{2}$. The SDW enhancement is relatively stronger around the vortex cores, which should give rise to the enhancement in $1 / T_{2}$ in this region; this agrees with the experimental observations in Ref. 15 . The analysis of our paper suggests that the difference in $1 / T_{2}$ will not become very large upon approaching the $\mathrm{SC}$ to SC+SDW boundary, as the SDW excitations become extended close to this phase boundary. As the magnetic field is increased, the SDW fluctuations should become more pronounced, so we expect that $1 / T_{2}$ will increase for all values of the local field. By contrast, in the BCS theory, one would expect that $1 / T_{2}$ decreases with increasing magnetic field, since the field becomes more uniform.

\section{ACKNOWLEDGMENTS}

We thank Gabriel Aeppli, Robert Birgeneau, Antonio Castro Neto, Rava da Silveira, Cristiane De Morais Smith, Seamus Davis, Mark Kastner, Boris Khaykovich, Steven Kivelson, Bella Lake, Kristine Lang, Young Lee, Andrew Millis, Anatoli Polkovnikov, Nick Read, Matthias Vojta, Jan Zaanen, and Shou-Cheng Zhang for numerous fruitful discussions. This research was supported by US NSF Grant No. DMR 0098226.

\section{APPENDIX A: DZYALOSHINSKII-MORIYA INTERACTION}

An orthorombic distortion of $\mathrm{La}_{2}{ }_{\delta} \mathrm{Sr}_{\delta} \mathrm{CuO}_{4}$ results in the Dzyaloshinskii-Moriya (DM) interaction for the $\mathrm{Cu}$ spins

$$
\mathcal{H}_{\mathrm{DM}}=\lambda \sum_{i, \delta}(-)^{i} \vec{d} \cdot \vec{S}_{i} \times \vec{S}_{i+\delta}
$$

where the sum over $\delta$ extends over all the nearest neighbors of site $i$, and $\vec{d}$ is a unit vector in the direction of the orthorombic a axis [i.e., a diagonal $(1,1)$ direction]. ${ }^{27}$ In this appendix, we study the effect of the DM interaction on the non-two-sublattice SDW, and for simplicity we consider a SDW at one wave vector only. The Hamiltonian (A1) mixes wave vectors $\mathbf{q}$ and $\mathbf{Q}+\mathbf{q}$, where $\mathbf{Q}=(\pi / a, \pi / a)$. In this case we need to modify Eq. (1.2) to

$$
\vec{S}(\mathbf{r}, \tau)=\operatorname{Re}\left[e^{i \mathbf{K}_{s x} \cdot \mathbf{r}} \vec{\Phi}_{x}(\mathbf{r}, \tau)+e^{i\left(\mathbf{K}_{s x}+\mathbf{Q}\right) \cdot \mathbf{r}} \vec{M}_{x}(\mathbf{r}, \tau)\right] .
$$

Straightforward algebra shows that the contribution of the DM interaction to the action is

$$
\widetilde{\mathcal{S}}_{D M}+\mathcal{S}_{M}=\int d^{2} d \tau\left\{\lambda\left(\mathbf{K}_{s}\right) \vec{d} \cdot\left[\vec{\Phi}_{x} \times \vec{M}_{x}^{*}+\text { c.c. }\right]+\frac{|\vec{M}|^{2}}{2 \chi}\right\}
$$

where $\lambda\left(\mathbf{K}_{s}\right)=2 \lambda\left[\cos \left(\mathbf{K}_{s x} \mathbf{a}_{x}\right)+\cos \left(\mathbf{K}_{s y} \mathbf{a}_{y}\right)\right]$ and the last term comes from the fact that spin fluctuations $\vec{M}_{x}$ are massive. We can now integrate $\vec{M}$ out, and find the anisotropy term for the SDW order parameter

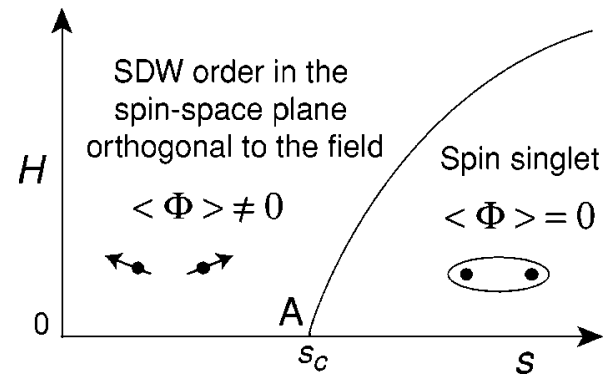

FIG. 32. Phase diagram of $\mathcal{S}_{\Phi}$ in Eq. (1.8) including the Zeeman coupling in Eq. (B1). The point A is the same as the corresponding point in Fig. 3. The central argument of Appendix B is that it requires a much larger field for $s>s_{c}$ near A to induce SDW order above, than in Fig. 3.

$$
\mathcal{S}_{D M}=-\frac{\lambda^{2}\left(\mathbf{K}_{s}\right) \chi}{2} \int d^{2} d \tau\left|\vec{\Phi}_{x} \times \vec{d}\right|^{2} .
$$

From Eq. (A4) we see that the DM interaction favors the collinear SDW, with direction of $\vec{\Phi}$ perpendicular to $\vec{d}$, i.e., along the orthorombic $\mathbf{b}$ axis (direction of the SDW ordering is always in the $\mathrm{CuO}$ plane). We also expect that the anisotropy becomes weaker with increasing doping due to a decrease of $\lambda\left(\mathbf{K}_{s}\right)$. However, the typical scale for the anisotropy is small, ${ }^{27}$ and so we expect that the quartic $u_{2}\left|\vec{\Phi}_{x}^{2}\right|^{2}$ term in Eq. (1.8) plays a dominant in selecting the collinear SDW at low temperatures. We note that $\mathcal{S}_{\mathrm{DM}}$ is quadratic, so it will favor the collinear SDW fluctuations even above the transition temperature.

\section{APPENDIX B: \\ ZEEMAN COUPLING TO THE MAGNETIC FIELD}

This appendix briefly discusses the effect of the Zeeman coupling to the magnetic field on the action $\mathcal{S}_{\Phi}$ in Eq. (1.8) for the SDW fluctuations. We will see that the effects are weaker than those considered in the body of the paper, especially near the critical point A at $s=s_{c}$ in zero field (see Fig. $3)$.

As reviewed in Ref. 121, in systems without an overdamped particle-hole continuum of spin excitations (as is the case here hear the ordering momenta $\mathbf{K}_{s x, y}$ ), we can deduce the coupling to the external field using simple gauge invariance arguments. In particular, the primary consequence of the external field is to rotate the spins uniformly about the field axis, and this can be accounted for by the following replacement to all temporal gradient terms

$$
\partial_{\tau} \Phi_{x \alpha} \rightarrow \partial_{\tau} \Phi_{\alpha}-i \epsilon_{\alpha \beta \gamma} H_{\beta} \Phi_{x \gamma},
$$

and similarly for $\Phi_{y \alpha}$. Here $H_{\alpha}$ is the three vector in spin space representing the external field. The resulting $\mathcal{S}_{\Phi}$ is closely related to models that have been studied in some detail $^{7,117}$ in the context of double layer quantum hall systems. From this work, we can deduce the phase diagram sketched in Fig. 32. The most important property of this phase diagram is that zero field phase transition at $s=s_{c}$ moves to finite field as $H \sim\left(s-s_{c}\right)^{z \nu}$ where the exponent 
$z \nu=1 / 2$ in mean-field theory. Fluctuation corrections will slightly increase this value, but the present critical field will nevertheless remain larger than the field in Eq. (3.9) associated with the corrections arising from the superflow. In particular, the phase boundary in Fig. 32 approaches the $H=0$ line with an infinite slope. Consequently, the Zeeman shift is subdominant to the stronger effects discussed in the body of the paper.

\section{APPENDIX C: \\ MICROSCOPIC THEORY FOR COUPLING BETWEEN SC AND SDW ORDER PARAMETERS}

In this appendix we discuss the microscopic origin of the effective interaction $\kappa$ between the SC and SDW order parameters in Eq. (1.10). We will argue that repulsive $\kappa>0$ is a remarkable property of doped Mott insulators, but weakly interacting electron systems quite possibly have $\kappa<0$.

We start by considering a weakly coupled Fermi liquid of electrons $c_{i \sigma}$ moving on the sites $i$ of a square lattice which is close to superconducting and commensurate antiferromagnetic instabilities

$$
\begin{gathered}
\mathcal{Z}=\int \mathcal{D} c^{\dagger} \mathcal{D} c e^{S[c]}, \\
S[c]=\int_{0}^{\beta} d \tau\left(\sum_{i} c_{i}^{\dagger} \partial_{\tau} c_{i}-\mathcal{H}[c]\right), \\
\mathcal{H}[c]=\sum_{k \sigma} \epsilon_{k} c_{k \sigma}^{\dagger} c_{k \sigma}+\mathcal{H}_{\mathrm{imp}}-\sum_{k}\left(\Delta_{k} c_{k \uparrow}^{\dagger} c_{k \downarrow}^{\dagger}+\text { H.c. }\right) \\
+\frac{\Delta_{0}^{2}}{2 \lambda_{\mathrm{SC}}}-\vec{\Phi} \sum_{k} c_{k+Q \alpha}^{\dagger} \vec{\sigma}_{\alpha \beta} c_{k \beta}^{\dagger}+\frac{\vec{\Phi}^{2}}{2 \lambda_{\mathrm{AF}}} .
\end{gathered}
$$

Here $\Delta_{k}=\Delta_{0}\left(\cos k_{x}-\cos k_{y}\right) / 2 \equiv \Delta_{0} d_{k}$ is the superconducting $d$-wave order parameter, and we assume a nearest-neighbor tight binding dispersion of the electrons $\epsilon_{k}=-2 t\left(\cos k_{x}\right.$ $\left.+\cos k_{y}\right)-\mu, Q=(\pi, \pi)$, and everywhere in this section momentum integrals go over the first Brillouin zone. $\mathcal{H}_{\text {imp }}$ describes the static potential of the impurities which gives rise to a finite quasiparticle lifetime

$$
\frac{1}{\tau}=2 \pi n_{\mathrm{imp}} N(0) V^{2},
$$

where $N(0)$ is the density of states on the fermi level and $V$ is the impurity potential.

Assuming that $\Delta_{0}$ and $\vec{\Phi}$ are small we can integrate out the fermions and obtain

$$
\begin{gathered}
\mathcal{Z}=e^{-\beta F}, \\
F=F_{\mathrm{GL}}\left[\Delta_{0}\right]+F_{\mathrm{AF}}[\vec{\Phi}]+\kappa\left|\Delta_{0}\right|^{2} \vec{\Phi}^{2} .
\end{gathered}
$$

The diagrammatic representations of the terms that contribute to $\kappa$ are shown on Fig. 33. Solid lines correspond to the quasiparticle propagators

$$
G\left(p, \omega_{n}\right)=\left[i \omega_{n}-\epsilon_{p}-i /(2 \tau) \operatorname{sgn}(\omega)\right],
$$

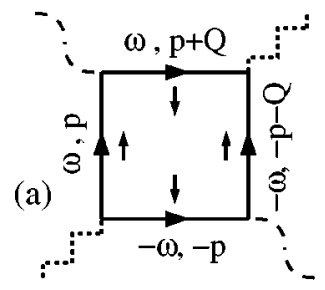

(b)

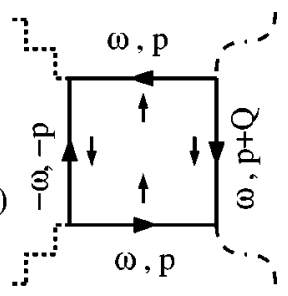

(c)

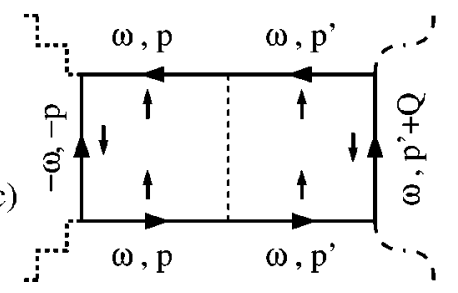

FIG. 33. Diagrams that contribute to the effective interaction between the superconducting and antiferromagnetic orders for Fermi liquids. Solid lines correspond to the quasiparticle propagators, zigzag lines correspond to the $d$-wave superconducting order parameter and contribute a factor $d_{p}$; the wavy line describe the SDW and a dashed line describes the static disorder potential.

zigzag lines correspond to the $d$-wave superconducting order parameter and contribute a factor $d_{p}$; wavy line describe the SDW, and dashed line describes the static disorder potential. We have

$$
\begin{gathered}
(\mathrm{a})=\frac{1}{\beta} \int \frac{d^{2} p}{(2 \pi)^{2}} d_{p} d_{p+Q} \sum_{\omega_{n}} G\left(\omega_{n}, p\right) G\left(-\omega_{n},-p\right) \\
\times \\
(\mathrm{b})=\frac{1}{\beta} \int \frac{d^{2} p}{(2 \pi)^{2}} d_{p}^{2} \sum_{\omega_{n}} G\left(\omega_{n}, p\right) G\left(-\omega_{n},-p\right) \\
\left.\quad \times G\left(\omega_{n}, p+Q\right) G\left(-\omega_{n},-p-Q\right), Q\right), \\
\text { (c) }=n_{\mathrm{imp}} V^{2} \frac{1}{\beta} \sum_{\omega_{n}} L\left(\omega_{n}\right) M\left(\omega_{n}\right)
\end{gathered}
$$

with

$$
\begin{gathered}
L\left(\omega_{n}\right)=\int \frac{d^{2} p}{(2 \pi)^{2}} d_{p}^{2} G\left(\omega_{n}, p\right) G\left(-\omega_{n},-p\right) G\left(\omega_{n}, p\right), \\
M\left(\omega_{n}\right)=\int \frac{d^{2} p}{(2 \pi)^{2}} G\left(\omega_{n}, p\right) G\left(-\omega_{n},-p\right) G\left(\omega_{n}, p+Q\right) .
\end{gathered}
$$

It is useful to note that if we define the static spin susceptibility at momentum $Q$ in the superconducting state

$$
\begin{aligned}
\chi(Q)= & -\frac{1}{\beta} \sum_{\omega_{n}} \int \frac{d^{2} p}{(2 \pi)^{2}}\left\{G_{s c}\left(\omega_{n}, p\right) G_{s c}\left(\omega_{n}, p+Q\right)\right. \\
& \left.+F\left(\omega_{n}, p\right) F\left(\omega_{n}, p+Q\right)\right\},
\end{aligned}
$$


with the Green's functions in the superconducting state defined in the usual manner, ${ }^{122}$ then

$$
\kappa=-\left.\frac{\partial^{2} \chi(Q)}{\partial \Delta_{0}^{*} \partial \Delta_{0}}\right|_{\Delta_{0}=0}
$$

which agrees with Eqs. (B6) and (B7).

In the limit $\mu \tau \gg 1$ the main contribution to $\kappa$ comes from the diagram (a) and we find for $T \rightarrow 0$

$$
\begin{aligned}
\kappa & =-\pi\left\langle d_{p}^{2}\right\rangle N(0) \frac{1}{\beta} \sum_{n>0} \frac{1}{\left(\omega_{n}+1 / 2 \tau\right)\left[\mu^{2}+\left(\omega_{n}+1 / 2 \tau\right)^{2}\right]} \\
& \approx-\frac{1}{2 \mu^{2}} \ln (\mu \tau) .
\end{aligned}
$$

It is important to note that in deriving the expression (C9) we relied on the fact that we have a $d$-wave superconductor with $d_{p+Q}=-d_{p}$ and took the average value of $\left\langle d_{p}^{2}\right\rangle$ on the Fermi surface to be 1 . Hence, such Fermi liquids on the square lattice have an effective "attraction" between the antiferromagnetic and superconducting orders, which can be traced back to the enhancement of the antiferromagnetic susceptibility (C7) in the $d$-wave superconducting state.

We have so far examined the interplay between SC and SDW orders near the boundary of their instability to a weakly interacting Fermi liquid. Now let us turn to the same interplay, but in the vicinity of a Mott insulator. Strong interactions are required to produce the Mott insulator, and so the perturbative approach of Eq. (C1) cannot be directly applied. Instead, we have to turn to alternative strong coupling approaches, in which the existence of the Mott insulator is built in at the outset. Such approaches have been discussed recently, and these are expressed in terms of collective degrees of freedom which are natural in the vicinity of of Mott insulator. Electron spin singlet states, spin one magnons, Cooper pairs of holes, and fermionic quasiparticles are introduced as individual excitations, and interactions between them are obtained from the microscopic $t-J$ Hamiltonian ${ }^{51,123}$ (phenomenological models of just the bosonic degrees of freedom have also been considered ${ }^{107}$ ). All these papers find strong repulsion between magnon and hole pair states, arising from the constraint on the allowed Hilbert space. The origin of this repulsion therefore lies in the short distance, lattice-scale physics of allowed low-energy states near a Mott insulator, rather than effects near the Fermi surface in the weak-coupling analysis discussed earlier. The Cooper pair-magnon repulsion immediately implies repulsion between the superconducting and antiferromagnetic orders, since the superconducting and antiferromagnetic phases correspond to the condensates of the corresponding particles. As an example, see Fig. 2 in Ref. 51: the pairing amplitude is weak in the region with magnetic order, but rises rapidly once the magnetic order is suppressed.

\section{APPENDIX D: RENORMALIZATION GROUP ANALYSIS OF COMPLEX VECTOR FIELDS}

This appendix will briefly review existing theoretical results for the critical properties of field theories which are similar to $\mathcal{S}_{\Phi}$, but simpler. The analysis of the full $\mathcal{S}_{\Phi}$ theory will be addressed in future work.

The simplification made here is to consider a field theory with only one complex vector field $\Phi_{\alpha}$, with $\alpha=1 \cdots m$; the original model has two such fields $\Phi_{x \alpha}$ and $\Phi_{y, \alpha}$. For the case of only one such field, we can always rescale $x$ and $y$ coordinates to make all velocities unity; then in $d$ space dimensions we are interested in the field theory with action

$$
\begin{aligned}
\mathcal{S}_{c}= & \int d^{d} r d \tau\left[\left|\partial_{\tau} \Phi_{\alpha}\right|^{2}+\left|\nabla_{r} \Phi_{\alpha}\right|^{2}+s\left|\Phi_{\alpha}\right|^{2}+\frac{u_{1}}{2}\left|\Phi_{\alpha}\right|^{4}\right. \\
& \left.+\frac{u_{2}}{2}\left|\Phi_{\alpha}^{2}\right|^{2}\right]
\end{aligned}
$$

This theory has upper critical dimension $d=3$, and can be studied in an expansion in $\epsilon=3-d$. Renormalization group equations for the quartic terms were obtained to $O\left(\epsilon^{2}\right)$ by Jones et al. ${ }^{55-57}$

$$
\begin{aligned}
\frac{d u_{1}}{d l}= & \epsilon u_{1}-K_{d}\left[(m+4) u_{1}^{2}+4 u_{1} u_{2}+4 u_{2}^{2}\right] \\
& +2 K_{d}^{2}\left[\frac{3}{2}(3 m+7) u_{1}^{3}+22 u_{1}^{2} u_{2}\right. \\
& \left.+(5 m+24) u_{1} u_{2}^{2}+4(m+2) u_{2}^{3}\right], \\
\frac{d u_{2}}{d l}= & \epsilon u_{2}-K_{d}\left[m u_{2}^{2}+6 u_{1} u_{2}\right] \\
& -2 K_{d}^{2}\left[(m-4) u_{2}^{3}-2(5+3 m) u_{2}^{2} u_{1}\right. \\
& \left.-\frac{1}{2}(5 m+41) u_{2} u_{1}^{2}\right],
\end{aligned}
$$

where $K_{d}=2^{-d+1 /[}\left[\pi^{d / 2} \Gamma(d / 2)\right]$. These flow equations always have two unstable fixed points: the Gaussian point $u_{1}^{*}$ $=u_{2}^{*}=0$ and the isotropic $\mathrm{O}(2 m)$ Heisenberg fixed point

$$
\begin{gathered}
u_{1}^{*}=\frac{\epsilon}{K_{d}} \frac{1}{m+4}+O\left(\epsilon^{2}\right), \\
u_{2}^{*}=0 .
\end{gathered}
$$

For sufficiently large or small $m$ there may also be two other fixed points

$$
\begin{gathered}
u^{*}=\frac{\epsilon}{6 K_{d}} B_{m}\left[3 m^{2}-12 m+144 \mp 3 m R_{m}^{1 / 2}\right] \epsilon+O\left(\epsilon^{2}\right), \\
v^{*}=\frac{\epsilon}{K_{d}} B_{m}\left[m^{2}+m-12 \pm 3 R_{m}^{1 / 2}\right] \epsilon+O\left(\epsilon^{2}\right),
\end{gathered}
$$


where $B_{m}^{-1}=m^{3}+4 m^{2}-24 m+144$ and $R_{m}=m^{2}-24 m$ +48 . The last two fixed points are absent in the case of $m$ $=3$. We note, however, that for $m=2$ and in the large $m$ limit a stable fixed point (the so-called chiral fixed point, see Ref. 57) is possible for $u_{2}>0$, so it may control the transition to the spiral order. When $u_{2}<0$, the system always flows towards strong coupling $u_{2} \rightarrow-\infty$, so we expect the transition to collinear order to be weakly first order.

\section{APPENDIX E:}

\section{NUMERICAL SOLUTION IN THE SC PHASE}

We will use the methods and notation described in Brandt. ${ }^{124}$ First, assume we know $\mathcal{V}_{H}(\mathbf{r})$. Write its Fourier expansion in the form

$$
\mathcal{V}_{H}(\mathbf{r})=\sum_{\mathbf{G}} d_{\mathbf{G}} e^{i \mathbf{G} \cdot \mathbf{r}}
$$

where $d_{\mathbf{G}}=d_{-\mathbf{G}}$ are both real, and $\mathbf{G}$ are the reciprocal lattice vectors of the triangular vortex lattice. Unlike the convention followed by Brandt, the sum over $\mathbf{G}$ always includes $\mathbf{G}$ $=0$, unless stated otherwise explicitly. In Brandt's notation, Eq. (E1) can be inverted by $d_{\mathbf{G}}=\left\langle\mathcal{V}_{H}(\mathbf{r}) \cos (\mathbf{G} \cdot \mathbf{r})\right\rangle$, where the angular bracket denotes a spatial average. Because of the symmetry we can work on only half a unit cell of the vortex lattice, and for simplicity we choose the half unit cell to be the one plotted in Fig. 13.

To obtain $G_{H}$, we want all the eigenvalues and eigenfunctions of the Schrödinger equation (4.20). As in the usual Bloch theory, these are labeled by a wave vector $\mathbf{k}$ in the first Brillouin zone, and a band index $\mu$. The explicit form of these are

$$
\Xi_{\mu \mathbf{k}}(\mathbf{r})=\frac{e^{i \mathbf{k} \cdot \mathbf{r}}}{\sqrt{A_{\mathcal{U}}}} \sum_{\mathbf{G}} c_{\mu \mathbf{G}}(\mathbf{k}) e^{i \mathbf{G} \cdot \mathbf{r}},
$$

where $A_{\mathcal{U}}$ is the area of the unit cell, and the $c_{\mu \mathbf{G}}(\mathbf{k})$ are normalized so that

$$
\sum_{\mathbf{G}}\left|c_{\mu \mathbf{G}}(\mathbf{k})\right|^{2}=1
$$

If we choose $M$ values of $\mathbf{G}$ (also as in Brandt), then $\mu=1$ $\cdots M$, and the $c_{\mu \mathbf{G}}(\mathbf{k})$ are the orthonormal eigenvalues of the $M \times M$ matrix $\mathcal{M}_{\mathbf{G}, \mathbf{G}^{\prime}}(\mathbf{k})$ where

$$
\begin{gathered}
\sum_{\mathbf{G}^{\prime}} \mathcal{M}_{\mathbf{G}, \mathbf{G}^{\prime}}(\mathbf{k}) c_{\mu \mathbf{G}^{\prime}}(\mathbf{k})=E_{\mu}^{2}(\mathbf{k}) c_{\mu \mathbf{G}}(\mathbf{k}), \\
\mathcal{M}_{\mathbf{G}, \mathbf{G}^{\prime}}(\mathbf{k})=(\mathbf{k}+\mathbf{G})^{2} \delta_{\mathbf{G}, \mathbf{G}^{\prime}}+d_{\mathbf{G}-\mathbf{G}^{\prime}} .
\end{gathered}
$$

After this diagonalization we obtain the Fourier components of Eq. (E1) as

$$
\begin{aligned}
d_{\mathbf{0}}= & s-s_{c}+\kappa\left(\sum_{\mathbf{G}} a_{\mathbf{G}}-1\right)+\frac{N u}{N_{k} A_{\mathcal{U}}} \\
& \times \sum_{\mathbf{k}, \mathbf{G}}\left[\frac{\operatorname{coth}\left[E_{\mathbf{G}}(\mathbf{k}) /(2 T)\right]}{E_{\mathbf{G}}(\mathbf{k})}-\frac{1}{\sqrt{(\mathbf{k}+\mathbf{G})^{2}+\Delta_{0}^{2}}}\right],
\end{aligned}
$$

and for $\mathbf{G} \neq 0$

$$
\begin{aligned}
d_{\mathbf{G}}= & -\kappa a_{\mathbf{G}}+\frac{N u}{2 N_{k} A_{\mathcal{U}}} \sum_{\mathbf{k}, \mathbf{G}^{\prime}, \mu} c_{\mu \mathbf{G}^{\prime}}\left[c_{\mu\left(\mathbf{G}^{\prime}+\mathbf{G}\right)}(\mathbf{k})\right. \\
& \left.+c_{\mu\left(\mathbf{G}^{\prime}-\mathbf{G}\right)}(\mathbf{k})\right] \frac{\operatorname{coth}\left[E_{\mu}(\mathbf{k}) /(2 T)\right]}{E_{\mu}(\mathbf{k})},
\end{aligned}
$$

where the sum over $\mathbf{k}$ is over $N_{k}$ points which average over the first Brillouin zone. Also note that $c_{\mu \mathbf{G}}(\mathbf{k})$ $=c_{\mu, \mathcal{R}(\mathbf{G})}[\mathcal{R}(\mathbf{k})]$, where $\mathcal{R}$ denotes a rotation by $\pi / 3$. This can be used to cut the number of $\mathbf{k}$ points in $1 / 6$.

The iteration of Eqs. (E5) and (E6) will produce the solution to Eq. (4.17) for a given $\psi_{H}(\mathbf{r})$. The next step is to solve Eq. (4.18), given $\mathcal{V}_{H}(\mathbf{r})$ in Eq. (E1). This is done just as in Brandt. His Eq. (9) is replaced by

$$
\begin{aligned}
\left(-\nabla_{\mathbf{r}}^{2}+2\right) \omega= & 2\left\{\left[1+\left(1-\frac{\kappa^{2}}{4 u \Upsilon}\right)\left|\psi_{0}\right|^{2}-\frac{\kappa}{4 u \Upsilon}\left(\mathcal{V}_{H}-\Delta_{0}^{2}\right)\right] \omega\right. \\
& \left.-\left(1-\frac{\kappa^{2}}{4 u \Upsilon}\right) \omega^{2}-\omega Q^{2}-g\right\}
\end{aligned}
$$

and a corresponding change to Brandt's Eq. (11). The new form of Brandt's Eq. (12) is

$a_{\mathbf{G}}:=a_{\mathbf{G}}$

$$
\times \frac{\left\langle\left[\left(1-\frac{\kappa^{2}}{4 u \Upsilon}\right)\left|\psi_{0}\right|^{2}-\frac{\kappa}{4 u \Upsilon}\left(\mathcal{V}_{H}-\Delta_{0}^{2}\right)\right] \omega-\omega Q^{2}-g\right\rangle}{\left\langle\omega^{2}\right\rangle\left[1-\kappa^{2} /(4 u \Upsilon)\right]} .
$$

After determining $\omega$ from above, we use this result to obtain new $\mathcal{V}_{H}(\mathbf{r})$ by solving Eq. (4.17), and so on. By iteration of Eqs. (4.17) and (4.18), we will be able to have the final solution to both of them.

Note that in order to get our numerical results, we did use a finite momentum cutoff. However, the equations have been designed to be cutoff independent and we did find that the Fourier components of $\psi_{H}(\mathbf{r})$ and $\mathcal{V}_{H}(\mathbf{r})$ decreases rapidly upon going to higher momenta.

\section{APPENDIX F: SPIN ORDERING PHASE BOUNDARY NEAR $M$}

Here we discuss the analytical solution of Eqs. (4.17) and (4.18) in the vicinity of the multi-critical point $M$ in Fig. 3, with the aim of determining the location of the AM phase boundary in its vicinity. Analytical progress is possible because the amplitude of the superconducting order $\left|\psi_{H}(\mathbf{r})\right|^{2}$ is small in this region. Our analysis will show that in this re- 
gion AM behaves as $H=1-\varrho\left(\kappa-s+s_{c}\right)$, where $\varrho$ is a numerical constant. The earlier full numerical solution in Sec. IV C led to the estimate $\varrho \approx 1.2$, and we shall find a consistent result here.

In addition to Eq. (E1), we use the Fourier expansions

$$
\begin{gathered}
T \sum_{\omega_{n}} G_{H}\left(\mathbf{r}, \mathbf{r}, \omega_{n}\right)-\int \frac{d \omega d^{2} k}{8 \pi^{3}} \frac{1}{\omega^{2}+k^{2}+\Delta_{0}^{2}}=\sum_{\mathbf{G}} b_{\mathbf{G}} e^{i \mathbf{G} \cdot \mathbf{r}}, \\
\left|\psi_{H}(\mathbf{r})\right|^{2}=\sum_{\mathbf{G}} a_{\mathbf{G}} e^{i \mathbf{G} \cdot \mathbf{r}} .
\end{gathered}
$$

Note that this notation for $a_{\mathbf{G}}$ is slightly different from that above and in Brandt.

Then Eq. (4.17) becomes

$$
\begin{gathered}
d_{0}=\Delta_{0}^{2}+\kappa\left(a_{0}-\left|\psi_{0}\right|^{2}\right)+2 N u b_{0}, \\
d_{\mathbf{G}}=\kappa a_{\mathbf{G}}+2 N u b_{\mathbf{G}}, \quad \mathbf{G} \neq 0 .
\end{gathered}
$$

Second, we can solve Eq. (4.6) by a Feynman graph expansion in $d_{\mathbf{G} \neq 0}$. This yields

$$
\begin{aligned}
b_{0} & =\int_{0}^{\infty} \frac{k d k}{2 \pi}\left[\frac{\operatorname{coth}\left(\sqrt{k^{2}+d_{0}} / 2 T\right)}{2 \sqrt{k^{2}+d_{0}}}-\frac{1}{2 \sqrt{k^{2}+\Delta_{0}^{2}}}\right]+\mathcal{O}\left(d_{\mathbf{G} \neq 0}^{2}\right) \\
& =\frac{\sqrt{d_{0}}-\Delta_{0}}{4 \pi}+\mathcal{O}\left(d_{\mathbf{G} \neq 0}^{2}\right) \text { at } T=0
\end{aligned}
$$

and

$$
\begin{aligned}
b_{\mathbf{G}}= & -\frac{d_{\mathbf{G}}}{4 \pi^{2}} \int_{0}^{\infty} \frac{d^{2} k}{(\mathbf{k}+\mathbf{G})^{2}-k^{2}}\left[\frac{\operatorname{coth}\left(\sqrt{k^{2}+d_{0}} / 2 T\right)}{2 \sqrt{k^{2}+d_{0}}}\right. \\
& \left.-\frac{\operatorname{coth}\left[\sqrt{(\mathbf{k}+\mathbf{G})^{2}+d_{0}} / 2 T\right]}{2 \sqrt{(\mathbf{k}+\mathbf{G})^{2}+d_{0}}}\right]+\mathcal{O}\left(d_{\mathbf{G} \neq 0}^{2}\right) \\
= & -\frac{d_{\mathbf{G}}}{8|\mathbf{G}|}+\mathcal{O}\left(d_{\mathbf{G} \neq 0}^{2}\right) \quad \text { at } T=0, \quad \mathbf{G} \neq 0 .
\end{aligned}
$$

Now we can solve Eqs. (F3),(F4),(F5) for the $d_{\mathbf{G}}$ in terms of the $a_{\mathbf{G}}$.

Finally, we need to determine the $a_{\mathbf{G}}$ by solving Eq. (4.18). This can be done with the realization that for small $\psi_{H}$, the functional form of the superconducting order parameter can be assumed to be equal to the Abrikosov solution. So we assume

$$
a_{\mathbf{G}}=-\frac{a_{0} a_{\mathbf{G}}^{A}}{2}, \quad \mathbf{G} \neq 0
$$

where $a_{\mathbf{G}}^{A}$ is given in Eq. (8) of Brandt. Now, it remains to obtain a single additional equation to determine $a_{0}$. This we determine by multiplying Eq. (4.18) by $\psi_{H}^{*}(\mathbf{r})$ and averaging over all space. Using the property of the Abrikosov solution for $\psi_{H}(\mathbf{r})$, we obtain

$$
\begin{aligned}
\left(1-\frac{\kappa^{2}}{4 u \Upsilon}\right) & \left(\sum_{\mathbf{G}} a_{\mathbf{G}} a_{-\mathbf{G}}-\left|\psi_{0}\right|^{2} a_{0}\right) \\
& +\frac{\kappa}{4 u \Upsilon}\left(\sum_{\mathbf{G}} d_{\mathbf{G}} a_{-\mathbf{G}}-\Delta_{0}^{2} a_{0}\right)-H a_{0}=0 .
\end{aligned}
$$

Equations (F3)-(F7) are now simple equations that can be easily solved to obtain all the Fourier coefficients. The line AM corresponds to $d_{0}=0$. Our analytical result of the slope of AM near M point is $\varrho \approx 1.1$, which is in acceptable agreement with that obtained from the full numerical solution.

\section{APPENDIX G:}

\section{NUMERICAL SOLUTION IN THE SC+SDW PHASE}

Here we will describe the solution of Eqs. (5.7), (5.4), and (5.8) for the unknowns $\mathcal{V}_{H}(\mathbf{r}), \psi_{H}(\mathbf{r})$, and $n_{H}(\mathbf{r})$. First, as Eq. (5.4) is linear in $n_{H}(\mathbf{r})$, it is convenient to rescale

$$
n_{H}(\mathbf{r}) \rightarrow n_{H}(\mathbf{r}) / \sqrt{2 N u},
$$

and these equations become

$$
\begin{aligned}
& \mathcal{V}_{H}(\mathbf{r})= s-s_{c}+\kappa\left[\left|\psi_{H}(\mathbf{r})\right|^{2}-1\right]+n_{H}^{2}(\mathbf{r}) \\
&+2 N u\left[T \sum_{\omega_{n}} G_{H}\left(\mathbf{r}, \mathbf{r}, \omega_{n}\right)-\int \frac{d \omega d^{2} k}{8 \pi^{3}} \frac{1}{\omega^{2}+k^{2}}\right], \\
& {\left[-\nabla_{\mathbf{r}}^{2}+\mathcal{V}_{H}(\mathbf{r})\right] n_{H}(\mathbf{r})=0, } \\
& {\left[\left(1-\frac{\kappa^{2}}{4 u \Upsilon}\right)\left[\left|\psi_{H}(\mathbf{r})\right|^{2}-1\right]+\frac{\kappa}{4 u \Upsilon}\left[\mathcal{V}_{H}(\mathbf{r})-s+s_{c}\right]\right.} \\
&\left.-\left(\nabla_{\mathbf{r}}-i \mathbf{A}\right)^{2}\right] \psi_{H}(\mathbf{r})=0 .
\end{aligned}
$$

We use two-step iteration to self-consistently solve the equations (G2, G3, G4). The first step consists of solving Eq. (G2) and (G3), and the second step is solving (G4).

For the first step, we use a four-substep iteration. First, define and calculate

$$
\begin{aligned}
\eta_{H}(\mathbf{r})= & s-s_{c}+\kappa\left[\left|\psi_{H}(\mathbf{r})\right|^{2}-1\right] \\
& +2 N u\left[T \sum_{\omega_{n}} G_{H}\left(\mathbf{r}, \mathbf{r}, \omega_{n}\right)-\int \frac{d \omega d^{2} k}{8 \pi^{3}} \frac{1}{\omega^{2}+k^{2}}\right] .
\end{aligned}
$$

Second, define and calculate the inverse of operator

$$
\mathcal{A}=-\nabla_{\mathbf{r}}^{2}+\eta_{H}(\mathbf{r}) .
$$

Third, calculate $n_{H}(\mathbf{r})$ which satisfies

$$
n_{H}(\mathbf{r})=-\mathcal{A}^{-1} n_{H}^{3}(\mathbf{r}) \text {. }
$$

Last, calculate $\mathcal{V}_{H}(\mathbf{r})$ using

$$
\mathcal{V}_{H}(\mathbf{r})=\eta_{H}(\mathbf{r})+n_{H}^{2}(\mathbf{r}) .
$$


Choosing proper initial value for $\mathcal{V}_{H}(\mathbf{r})$ and $n_{H}(\mathbf{r})$ and iterate Eqs. (G5), (G6), (G7), (G8) will produce the solution to both Eqs. (G2) and (G3).

In practice, the above steps are performed in momentum space. If we let

$$
\begin{aligned}
& \mathcal{V}_{H}(\mathbf{r})=\sum_{\mathbf{G}} d_{\mathbf{G}} e^{i \mathbf{G} \cdot \mathbf{r}}, \\
& n_{H}(\mathbf{r})=\sum_{\mathbf{G}} \widetilde{f}_{\mathbf{G}} e^{i \mathbf{G} \cdot \mathbf{r}}, \\
& \eta_{H}(\mathbf{r})=\sum_{\mathbf{G}} g_{\mathbf{G}} e^{i \mathbf{G} \cdot \mathbf{r}},
\end{aligned}
$$

where $\mathbf{G}$ are the reciprocal lattice vectors of the vortex lattice [note that $\widetilde{f}_{\mathbf{G}}$ differs slightly from $f_{\mathbf{G}}$ in Eq. (5.10) because of the rescaling Eq. (G1)], then Eqs. (G5)-(G8) become

$$
\begin{aligned}
& g_{\mathbf{0}}=s-s_{c}+\kappa\left(\sum_{\mathbf{G}} a_{\mathbf{G}}-1\right) \\
& +\frac{N u}{N_{k} A_{\mathcal{U}}} \sum_{\mathbf{k}+\mathbf{G} \neq 0}\left[\frac{\operatorname{coth}\left[E_{\mathbf{G}}(\mathbf{k}) /(2 T)\right]}{E_{\mathbf{G}}(\mathbf{k})}-\frac{1}{|\mathbf{k}+\mathbf{G}|}\right],
\end{aligned}
$$

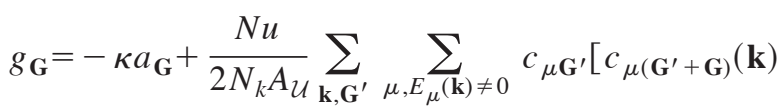

$$
\begin{aligned}
& \left.+c_{\mu\left(\mathbf{G}^{\prime}-\mathbf{G}\right)}(\mathbf{k})\right] \frac{\operatorname{coth}\left[E_{\mu}(\mathbf{k}) /(2 T)\right]}{E_{\mu}(\mathbf{k})},
\end{aligned}
$$

*Electronic address: ying.zhang@yale.edu

†Electronic address: demler@cmts.harvard.edu

Electronic address: subir.sachdev@yale.edu; URL: http:// pantheon.yale.edu/ $\sim$ subir

${ }^{1}$ S. Wakimoto, G. Shirane, Y. Endoh, K. Hirota, S. Ueki, K. Yamada, R.J. Birgeneau, M.A. Kastner, Y.S. Lee, P.M. Gehring, and S.H. Lee, Phys. Rev. B 60, R769 (1999).

${ }^{2}$ S. Wakimoto, R.J. Birgeneau, Y.S. Lee, and G. Shirane, Phys. Rev. B 63, 172501 (2001).

${ }^{3}$ G. Aeppli, T.E. Mason, S.M. Hayden, H.A. Mook, and J. Kulda, Science 278, 1432 (1997).

${ }^{4}$ S. Sachdev, Science 288, 475 (2000).

${ }^{5}$ J.L. Tallon and J.W. Loram, Physica C 349, 53 (2001).

${ }^{6}$ T. Valla, A.V. Fedorov, P.D. Johnson, B.O. Wells, S.L. Hulbert, Q. Li, G.D. Gu, and N. Koshizuka, Science 285, 2110 (1999).

${ }^{7}$ S. Sachdev, Quantum Phase Transitions (Cambridge University Press, Cambridge, 1999).

${ }^{8}$ S. Sachdev, C. Buragohain, and M. Vojta, Science 286, 2479 (1999); M. Vojta, C. Buragohain and S. Sachdev, Phys. Rev. B 61, 15152 (2000).

${ }^{9}$ E. Demler, S. Sachdev, and Y. Zhang, Phys. Rev. Lett. 87, 067202 (2001).

${ }^{10}$ S. Sachdev, cond-mat/0108238, J. Phys. Chem. Solids (to be published).

${ }^{11}$ A. Polkovnikov, S. Sachdev, M. Vojta, and E. Demler, Proceed-

$$
\begin{gathered}
\mathcal{A}_{\mathbf{G G}^{\prime}}=\mathbf{G}^{2} \delta_{\mathbf{G}, \mathbf{G}^{\prime}}+g_{\mathbf{G}^{-}-\mathbf{G}^{\prime}}, \\
\widetilde{f}_{\mathbf{G}}=-\sum_{\mathbf{G}^{\prime}} \mathcal{A}_{\mathbf{G G}^{\prime}}^{-1}\left\langle n_{H}^{3}(\mathbf{r}) \cos \left(\mathbf{G}^{\prime} \cdot \mathbf{r}\right)\right\rangle, \\
d_{\mathbf{G}}=g_{\mathbf{G}}+\left\langle n_{H}^{2}(\mathbf{r}) \cos \left(\mathbf{G}^{\prime} \cdot \mathbf{r}\right)\right\rangle .
\end{gathered}
$$

Note that in the substep (G13) the equation is solved by another smaller iteration.

The second step is very similar to the case with no magnetic order as in Appendix E. Eq. (G4) can be solved by a two-substep iteration of the following equations:

$$
\left(-\nabla_{\mathbf{r}}^{2}+2\right) \omega=2\left[[1+G(\mathbf{r})] \omega-\left(1-\frac{\kappa^{2}}{4 u \Upsilon}\right) \omega^{2}-\omega Q^{2}-g\right]
$$

where

$$
G(\mathbf{r})=\left(1-\frac{\kappa^{2}}{4 u \Upsilon}\right)-\frac{\kappa}{4 u \Upsilon}\left[\mathcal{V}_{H}(\mathbf{r})-s+s_{c}\right]
$$

and

$$
a_{\mathbf{G}}=a_{\mathbf{G}} \cdot \frac{\left\langle\omega G-\omega Q^{2}-g\right\rangle}{\left\langle\omega^{2}\right\rangle\left[1-\kappa^{2} /(4 u \Upsilon)\right]} .
$$

From the iteration results we are able to determine $\mathcal{V}_{H}(\mathbf{r})$, $n_{H}(\mathbf{r})$ and $\psi_{H}(\mathbf{r})$.

ings of PPHMF IV (World Scientific, Singapore, in press), condmat/0110329.

${ }^{12}$ B. Lake, G. Aeppli, K.N. Clausen, D.F. McMorrow, K. Lefmann, N.E. Hussey, N. Mangkorntong, M. Nohara, H. Takagi, T.E. Mason, and A. Schröder, Science 291, 1759 (2001).

${ }^{13}$ B. Khaykovich, Y.S. Lee, R.W. Erwin, S.-H. Lee, S. Wakimoto, K.J. Thomas, M.A. Kastner, and R.J. Birgeneau, Phys. Rev. B 66, 014528 (2002).

${ }^{14}$ B. Lake, H.M. Rønnow, N.B. Christensen, G. Aeppli, K. Lefmann, D.F. McMorrow, P. Vorderwisch, P. Smeibidl, N. Mangkorntong, T. Sasagawa, M. Nohara, H. Takagi, and T.E. Mason, Nature (London) 415, 299 (2002).

${ }^{15}$ N.J. Curro, C. Milling, J. Haase, and C.P. Slichter, Phys. Rev. B 62, 3473 (2000).

${ }^{16}$ V.F. Mitrović, E.E. Sigmund, M. Eschrig, H.N. Bachman, W.P. Halperin, A.P. Reyes, P. Kuhns, and W.G. Moulton, Nature (London) 413, 501 (2001).

${ }^{17}$ V.F. Mitrović, E.E. Sigmund, W.P. Halperin, A.P. Reyes, P. Kuhns, and W.G. Moulton, cond-mat/0202368 (unpublished).

${ }^{18}$ J.E. Hoffman, E.W. Hudson, K.M. Lang, V. Madhavan, S.H. Pan, H. Eisaki, S. Uchida, and J.C. Davis, Science 295, 466 (2002).

${ }^{19}$ J.M. Tranquada, J.D. Axe, N. Ichikawa, Y. Nakamura, S. Uchida, and B. Nachumi, Phys. Rev. B 54, 7489 (1996).

${ }^{20}$ Y.S. Lee, R.J. Birgeneau, M.A. Kastner, Y. Endoh, S. Wakimoto, K. Yamada, R.W. Erwin, S.-H. Lee, and G. Shirane, Phys. Rev. B 60, 3643 (1999). 
${ }^{21}$ A.H. Castro Neto and D. Hone, Phys. Rev. Lett. 76, 2165 (1996).

${ }^{22}$ J. Zaanen, Physica C 317, 217 (1999).

${ }^{23}$ O. Zachar, S.A. Kivelson, and V.J. Emery, Phys. Rev. B 57, 1422 (1998)

${ }^{24}$ D. Podolsky, E. Demler, K. Damle, and B.I. Halperin, cond-mat/0204011 (unpublished).

${ }^{25}$ M. Vojta, cond-mat/0204284 (unpublished); A. Polkovnikov, M. Vojta, and S. Sachdev, Phys. Rev. B 65, 220509 (2002).

${ }^{26}$ M. Vojta and S. Sachdev, Phys. Rev. Lett. 83, 3916 (1999); M. Vojta, Y. Zhang, and S. Sachdev, Phys. Rev. B 62, 6721 (2000). These papers studied models of $S=1 / 2$ fermions with $\operatorname{Sp}(N)$ symmetry in the large $N$ limit. This large $N$ limit is quite distinct from that used in the present paper, which applies instead to bosonic $S=1$ excitons.

${ }^{27}$ T. Thio and A. Aharony, Phys. Rev. Lett. 73, 894 (1994).

${ }^{28}$ S. Andergassen, S. Caprara, C. Di Castro, and M. Grilli, Phys. Rev. Lett. 87, 056401 (2001).

${ }^{29}$ A. Abanov and A.V. Chubukov, Phys. Rev. Lett. 84, 5608 (2000).

${ }^{30}$ S.A. Kivelson, E. Fradkin, and V.J. Emery, Nature (London) 393, 550 (1998).

${ }^{31}$ M. Granath, V. Oganesyan, S.A. Kivelson, E. Fradkin, and V.J. Emery Phys. Rev. Lett. 87, 167011 (2001).

${ }^{32}$ S.-C. Zhang, Science 275, 1089 (1997).

${ }^{33}$ D.P. Arovas, A.J. Berlinsky, C. Kallin, and S.-C. Zhang, Phys. Rev. Lett. 79, 2871 (1997).

${ }^{34}$ H. Bruus, K.A. Eriksen, M. Hallundbæk, and P. Hedegård, Phys. Rev. B 59, 4349 (1999).

${ }^{35}$ A.V. Chubukov, S. Sachdev, and J. Ye, Phys. Rev. B 49, 11919 (1994).

${ }^{36}$ Note that Eqs. (18) and (30) in Bruus et al. (Ref. 34) and the analogous Eq. (3) in Arovas et al. (Ref. 33) have zero on the right-hand side; this follows from the perspective of rotation of the static order parameter from SC to Néel. Consequently, Eqs. (23) and (31) in Bruus et al. (Ref. 34), which describe the spin excitations, have gapless Goldstone modes because static SDW is present. This should be contrasted to our Eq. (1.17), whose right-hand side is nonzero, and whose SDW spectrum is gapped.

${ }^{37}$ J.-P. Hu and S.-C. Zhang, cond-mat/0108273 (unpublished).

${ }^{38}$ J.A. Hertz, L. Fleishman, and P.W. Anderson, Phys. Rev. Lett. 43, 942 (1979).

${ }^{39}$ A.J. Bray and M.A. Moore, J. Phys. C 15, L765 (1982); J.W. Hartman and P.B. Weichman, Phys. Rev. Lett. 74, 4584 (1995).

${ }^{40}$ S. Sachdev, Phys. Rev. B 45, 389 (1992).

${ }^{41}$ N. Nagaosa and P.A. Lee, Phys. Rev. B 45, 966 (1992).

${ }^{42}$ M. Ogata, Int. J. Mod. Phys. B 13, 3560 (1999).

${ }^{43}$ X. Hu, cond-mat/9906237 (unpublished).

${ }^{44}$ M. Juneau, R. MacKenzie, M.-A. Vachon, and J.M. Cline, Phys. Rev. B 65, 140512 (2002).

${ }^{45}$ B.M. Andersen, H. Bruus, and P. Hedegård, Phys. Rev. B 61, 6298 (2000).

${ }^{46}$ J.H. Han and D.H. Lee, Phys. Rev. Lett. 85, 1100 (2000).

${ }^{47}$ M. Franz and Z. Tesanovic, Phys. Rev. B 63, 064516 (2001).

${ }^{48}$ J.-X. Zhu and C.S. Ting, Phys. Rev. Lett. 87, 147002 (2001).

${ }^{49}$ P.A. Lee and X.-G. Wen, Phys. Rev. B 63, 224517 (2001).

${ }^{50}$ J.I. Kishine, P.A. Lee, and X.-G. Wen, Phys. Rev. Lett. 86, 5365 (2001).

${ }^{51}$ K. Park and S. Sachdev, Phys. Rev. B 64, 184510 (2001); see the discussion in this paper in the second-to-last paragraph on $\mathrm{p}$. 184510-12.
${ }^{52}$ S.R. White and D.J. Scalapino, Phys. Rev. Lett. 80, 1272 (1998).

${ }^{53}$ Y. Chen and C.S. Ting, Phys. Rev. B 65, 180513 (2002).

${ }^{54}$ D.-H. Lee, Phys. Rev. Lett. 88, 227003 (2002).

${ }^{55}$ D. Jones, A. Love, and M.A. Moore, J. Phys. C 9, 743 (1976).

${ }^{56}$ D. Bailin, A. Love, and M.A. Moore, J. Phys. C 10, 1159 (1977).

${ }^{57}$ H. Kawamura, Phys. Rev. B 38, 4916 (1988); J. Phys.: Condens. Matter 10, 4707 (1998).

${ }^{58}$ M. Tissier, B. Delamotte, and D. Mouhanna, Phys. Rev. Lett. 84, 5208 (2000); cond-mat/0101167 (unpublished).

${ }^{59}$ M. Itakura, cond-mat/0110306 (unpublished).

${ }^{60}$ A.F. Andreev and I.A. Grishchuck, Sov. Phys. Solid State 66, 1088 (1984).

${ }^{61}$ A.V. Chubukov, J. Phys.: Condens. Matter 2, 1593 (1989).

${ }^{62}$ L.P. Gorkov and A.V. Sokol, JETP Lett. 52, 504 (1990); V. Barzykin, L.P. Gorkov, and A.V. Sokol, Europhys. Lett. 15, 869 (1991).

${ }^{63}$ E. Demler, C. Nayak, H.-Y. Kee, Y.-B. Kim, and T. Senthil, Phys. Rev. B 65, 155103 (2002).

${ }^{64}$ P.G. de Gennes and J. Prost, The Physics of Liguid Crystals (Oxford University Press, Oxford, 1995).

${ }^{65}$ E.F. Gramsbergen, L. Longa, and W.H. de Jeu, Phys. Rep. 135, 195 (1986).

${ }^{66}$ C.V. Ciobanu, S.-K. Yip, and T.-L. Ho, Phys. Rev. A 61, 033607 (2000).

${ }^{67}$ R.A. Jalabert and S. Sachdev, Phys. Rev. B 44, 686 (1991).

${ }^{68}$ P.E. Lammert, D.S. Rokhsar, and J. Toner, Phys. Rev. Lett. 70, 1650 (1993).

${ }^{69}$ T. Senthil and M.P.A. Fisher, Phys. Rev. B 62, 7850 (2000).

${ }^{70}$ E. Demler and F. Zhou, Phys. Rev. Lett. 88, 163001 (2002).

${ }^{71}$ S. Sachdev and K. Park, Ann. Phys. (N.Y.) 298, 58 (2002); K. Park and S. Sachdev, Phys. Rev. B 65, 220405 (2002).

${ }^{72}$ J. Zaanen, O.Y. Osman, H.V. Kruis, Z. Nussinov, and J. Tworzydlo, Philos. Mag. B 81, 1485 (2002).

${ }^{73}$ T. Senthil and M.P.A. Fisher, cond-mat/9912380 (unpublished).

${ }^{74}$ N. Kirova and S. Brazovskii, J. Phys. IV 10, 3 (2000).

${ }^{75}$ V.J. Emery, S.A. Kivelson, and J.M. Tranquada, Proc. Natl. Acad. Sci. U.S.A. 96, 8814 (1999).

${ }^{76}$ For completeness, we note that the corresponding homotopy group for the case with circular spiral $\operatorname{SDW}\left(u_{2}>0\right.$ in $\left.\mathcal{S}_{\Phi}\right)$ is $\pi_{1}\left(S_{3} / Z_{2}\right)=Z_{2}$.

${ }^{77}$ S.M. Hayden, G. Aeppli, H. Mook, D. Rytz, M.F. Hundley, and Z. Fisk, Phys. Rev. Lett. 66, 821 (1991).

${ }^{78}$ B. Keimer, N. Belk, R.J. Birgeneau, A. Cassanho, C.Y. Chen, M. Greven, M.A. Kastner, A. Aharony, Y. Endoh, R.W. Erwin, and G. Shirane, Phys. Rev. B 46, 14034 (1992).

${ }^{79}$ S. Sachdev and J. Ye, Phys. Rev. Lett. 69, 2411 (1992); A.V. Chubukov and S. Sachdev, ibid. 71, 169 (1993).

${ }^{80}$ A. Sokol and D. Pines, Phys. Rev. Lett. 71, 2813 (1993).

${ }^{81}$ T. Imai, C.P. Slichter, K. Yoshimura, and K. Kosuge, Phys. Rev. Lett. 70, 1002 (1993); T. Imai, C.P. Slichter, K. Yoshimura, M. Katoh, and K. Kosuge, ibid. 71, 1254 (1993).

${ }^{82}$ S. Fujiyama, M. Takigawa, Y. Ueda, T. Suzuki, and N. Yamada, Phys. Rev. B 60, 9801 (1999).

${ }^{83}$ S. Katano, M. Sato, K. Yamada, T. Suzuki, and T. Fukase, Phys. Rev. B 62, R14677 (2000).

${ }^{84}$ D. Vaknin, J. Zarestky, and L. Miller, Physica C 329, 109 (2000).

${ }^{85}$ R.I. Miller, R.F. Kiefl, J.H. Brewer, J.E. Sonier, J. Chakhalian, S. Dunsiger, G.D. Morris, A.N. Price, D.A. Bonn, W.H. Hardy, and R. Liang, Phys. Rev. Lett. 88, 137002 (2002). 
${ }^{86}$ C. Panagopoulos, B.D. Rainford, J.R. Cooper, C.A. Scott, and T. Xiang, cond-mat/0007158 (unpublished); C. Panagopoulos, B. D. Rainford, J. L. Tallon, T. Xiang, J. R. Cooper, and C. A. Scott (unpublished).

${ }^{87}$ J.E. Sonier, J.H. Brewer, R.F. Kiefl, R.H. Heffner, K. Poon, S.L. Stubbs, G.D. Morris, R.I. Miller, W.N. Hardy, R. Liang, D.A. Bonn, J.S. Gardner, and N.J. Curro, cond-mat/0108479.

${ }^{88}$ P. Bourges, Y. Sidis, H.F. Fong, L.P. Regnault, J. Bossy, A. Ivanov, and B. Keimer, Science 288, 1234 (2000).

${ }^{89}$ H.A. Mook, P. Dai, and F. Dogan, Phys. Rev. Lett. 88, 097004 (2002).

${ }^{90}$ C.D. Batista, G. Ortiz, and A.V. Balatsky, Phys. Rev. B 64, 172508 (2001).

${ }^{91}$ J. Rossat-Mignod, L.P. Regnault, C. Vettier, P. Bourges, P. Burlet, J. Bossy, J.Y. Henry, and G. Lapertot, Physica C 185-189, 86 (1991).

${ }^{92}$ H.A. Mook, M. Yethiraj, G. Aeppli, T.E. Mason, and T. Armstrong, Phys. Rev. Lett. 70, 3490 (1993).

${ }^{93}$ H.F. Fong, B. Keimer, D.L. Milius, and I.A. Aksay, Phys. Rev. Lett. 78, 713 (1997).

${ }^{94}$ E. Demler and S.-C. Zhang, Phys. Rev. Lett. 75, 4126 (1995).

${ }^{95}$ E. Demler, H. Kohno, and S.-C. Zhang, Phys. Rev. B 58, 5719 (1998).

${ }^{96}$ S. Rabello, H. Kohno, E. Demler, and S.-C. Zhang, Phys. Rev. Lett. 80, 3586 (1998).

${ }^{97}$ E. Demler and S.-C. Zhang, Ann. Phys. (N.Y.) 82, 3895 (1999).

${ }^{98}$ S. Meixner, W. Hanke, E. Demler, and S.-C. Zhang, Phys. Rev. Lett. 79, 4902 (1997).

${ }^{99}$ R. Eder, W. Hanke, and S.-C. Zhang, Phys. Rev. B 57, 13781 (1998).

${ }^{100}$ Y.B. Bazaliy, E. Demler, and S.-C. Zhang, Phys. Rev. Lett. 79, 1921 (1997).

${ }^{101}$ O. Tchernyshyov, M.R. Norman, and A.V. Chubukov, Phys. Rev. B 63, 144507 (2001).

${ }^{102}$ S. Sachdev and M. Vojta, Physica B 280, 333 (2000); S. Sachdev, Science 288, 475 (2000).

${ }^{103}$ M. Veillette, Y.B. Bazaliy, A.J. Berlinsky, and C. Kallin, Phys. Rev. Lett. 83, 2413 (1999).

${ }^{104}$ L.P. Pryadko, S.A. Kivelson, V.J. Emery, Y.B. Bazaliy, and E.A. Demler, Phys. Rev. B 60, 7541 (1999).

${ }^{105}$ K.-S. Liu and M.E. Fisher, J. Low Temp. Phys. 10, 655 (1972); J.M. Kosterlitz, D.R. Nelson, and M.E. Fisher, Phys. Rev. B 13, 412 (1976).
${ }^{106}$ The presence or absence of SO (5) symmetry in equal-time correlations of the classical theory of the finite temperature bicritical point has no implications for symmetries of dynamic quantum models for zero temperature transitions, contrary to claims in Ref. 32. Indeed, the dynamic fluctuations of the superconducting order parameter are overdamped at this finite temperature bicritical point, as are the $\pi$ excitations associated with rotations of the SC order into the SC direction-so there is no "resonance peak:" these conclusions follow directly from the classical critical dynamic theory of D.L. Huber and R. Raghavan, Phys. Rev. B 14, 4068 (1976); see also S. Murakami and N. Nagaosa, J. Phys. Soc. Jpn. 69, 2395 (2000).

${ }^{107}$ S.-C. Zhang, J.P. Hu, E. Arrigoni, W. Hanke, and A. Auerbach, Phys. Rev. B 60, 13070 (1999).

${ }^{108}$ S.A. Kivelson, G. Aeppli, and V.J. Emery, Proc. Natl. Acad. Sci. U.S.A. 98, 11903 (2001).

${ }^{109}$ A. Aharony, Phys. Rev. Lett. 88, 059703 (2002); P. Calabrese, A. Pelissetto, and E. Vicari, cond-mat/0203533 (unpublished).

${ }^{110}$ I. Martin, G. Ortiz, A. V. Balatsky, and A. R. Bishop, Int. J. Mod. Phys. 14, 3567 (2000).

${ }^{111}$ E. Demler, A.J. Berlinsky, C. Kallin, G.B. Arnold, and M.R. Beasley, Phys. Rev. Lett. 80, 2917 (1998).

${ }^{112}$ A.J. Berlinsky, G.B. Arnold, D. Arovas, M. Beasley, C. Kallin, and E. Demler, J. Phys. Chem. Solids 59, 1794 (1998).

${ }^{113}$ B.C. den Hertog, A.J. Berlinsky, and C. Kallin, Phys. Rev. B 59, R11645 (1999).

${ }^{114}$ D.E. Sheehy and P.M. Goldbart, Phys. Rev. B 57, R8131 (1998).

${ }^{115}$ P.M. Goldbart and D.E. Sheehy, Phys. Rev. B 58, 5731 (1998).

${ }^{116}$ R.S. Decca, H.D. Drew, E. Osquiguil, B. Maiorov, and J. Guimpel, Phys. Rev. Lett. 85, 3708 (2000).

${ }^{117}$ S. Das Sarma, S. Sachdev, and L. Zheng, Phys. Rev. Lett. 79, 917 (1997); Phys. Rev. B 58, 4672 (1998).

${ }^{118}$ G.S. Boebinger, Y. Ando, A. Passner, T. Kimura, M. Okuya, J. Shimoyama, K. Kishio, K. Tamasaku, N. Ichikawa, and S. Uchida, Phys. Rev. Lett. 77, 5417 (1996).

${ }^{119}$ R. Wortis, A.J. Berlinsky, and C. Kallin, Phys. Rev. B 61, 12342 (2000)

${ }^{120}$ D.K. Morr and R. Wortis, Phys. Rev. B 61, R882 (2000).

${ }^{121}$ S. Sachdev, Z. Phys. B: Condens. Matter 94, 469 (1994).

${ }^{122}$ G.D. Mahan, Many-Particle Physics (Plenum Press, New York, 1990).

${ }^{123}$ E. Altman and A. Auerbach, Phys. Rev. B 65, 104508 (2002).

${ }^{124}$ E.H. Brandt, Phys. Rev. Lett. 78, 2208 (1997). 